\title{
Fish Streams Along the Trans-Alaska Pipeline System
}

A Compilation of Selected References with Current TAPS Stationing

Third Edition

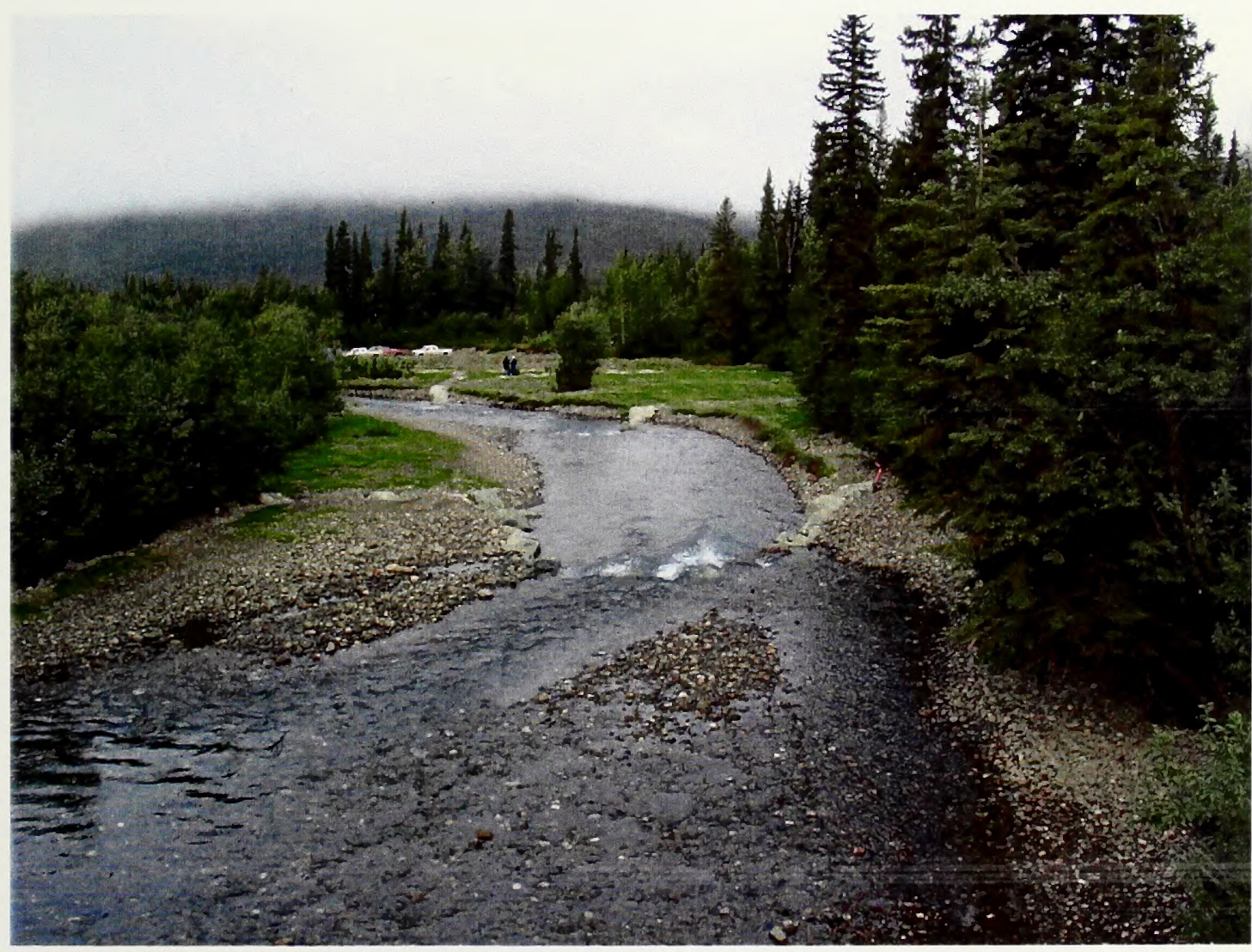




\section{The BLM Mission}

The Bureau of Land Management sustains the health, diversity and productivity of the public lands for the use and enjoyment of present and future generations.

\section{Cover Photo}

The stable stream channel of Marion Creek near milepost 180 Dalton Highway. Design constructed by Alyeska Pipeline Service Company in 2000. Photo by Dennis Gnath.

\section{Open File Reports}

Open File Reports issued by the Bureau of Land Management-Alaska present the results of inventories or other investigations on a variety of scientific and technical subjects that are made available to the public outside the formal BLM-Alaska technical publication series. These reports can include preliminary or incomplete data and are not published and distributed in quantity.

This report is available from the Office of Pipeline Monitoring, 411 West 4 th Avenue, Anchorage, Alaska 99501, telephone (907) 257-1300.

Other reports in the series are available while supplies last from BLM External Affairs, 222 West 7th Avenue \#13, Anchorage, Alaska 99513 and from the Juneau Minerals Information Center, 100 Savikko Road, Mayflower Island, Douglas, AK 99824, (907) 364-1553. Copies are also available for inspection at the Alaska Resource Library and Information Service (Anchorage), the USDI Resources Library in Washington, D. C., various libraries of the University of Alaska, the BLM National Business Center Library (Denver) and other selected locations.

A complete bibliography of all BLM-Alaska scientific reports can be found on the Internet at: http://www.ak.blm.gov/affairs/sci_rpts.html. Related publications are also listed athttp://juneau.ak.blm.gov. 


\section{Fish Streams Along the Trans-Alaska Pipeline System}

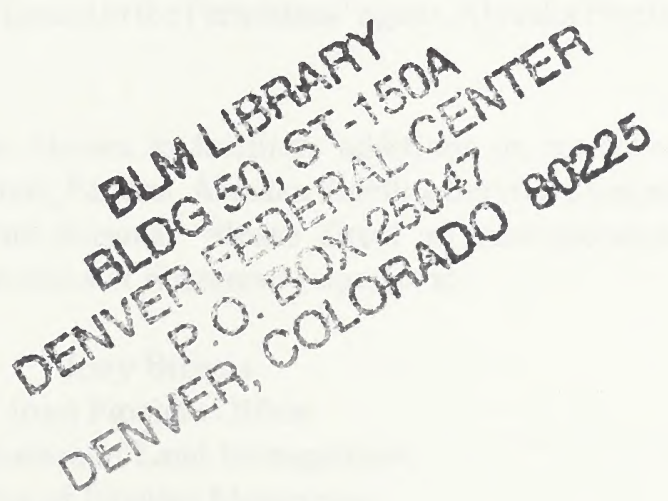




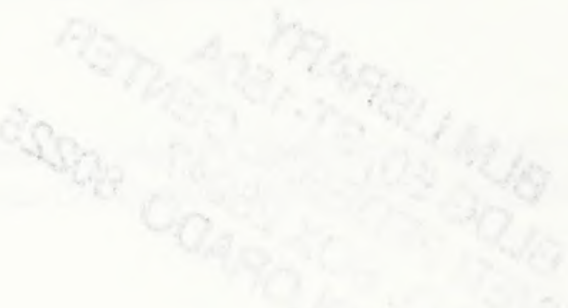




\section{Preface to the Third Edition}

This third edition of the Fish Streams Along the Trans-Alaska Pipeline System (previously titled TAPS Fish Streams) has been prepared and will be maintained by the Office of Pipeline Monitoring to facilitate the administration of environmental provisions of the Federal Agreement and Grant of Right-of-Way for the Trans-Alaska Pipeline System. Specifically, this report is intended to serve as the source document for periodically updating a list of areas under Federal administration where pipeline activities may have to be restricted to protect fish breeding, spawning and major migration. However, this report is not the Federal Authorized Officer's list required under the Agreement and Grant of Right-of-Way for Trans-Alaska Pipeline, Stipulation 2.5.3, Zones of Restricted Activities. The Stipulation 2.5.3 list will only include streams in areas of Federal pipeline administration and will be separately prepared and issued to the Permittees' agent, Alyeska Pipeline Service Company.

The open file format was chosen to facilitate additions or corrections resulting from any future State, Federal, Alyeska Pipeline Service Company or other studies of pipeline streams. Please direct all correspondence concerning additions/corrections or document requests to:

Jerry Brossia Joint Pipeline Office

U.S. Bureau of Land Management Office of Pipeline Monitoring

411 West $4^{\text {th }}$ Avenue Anchorage, Alaska 99501

(907) 257-1300 


\section{Fish Species Codes}

(Adapted from Johnson and Rockwell, 1981)

\begin{tabular}{|c|c|c|c|}
\hline$?$ & $=$ & Fish Present? & \\
\hline$A B$ & $=$ & Alaska blackfish & :Dallia pectoralis \\
\hline $\mathrm{AC}$ & $=$ & Arctic char & :Salvelinus alpinus \\
\hline $\mathrm{AL}$ & $=$ & Arctic lamprey & :Lampetra japonica \\
\hline AS & $=$ & American shad & : Alosa sapidissima \\
\hline $\mathrm{BB}$ & $=$ & Burbot & :Lota lota \\
\hline $\mathrm{BC}$ & $=$ & Bering cisco & : Coregonus laurettae \\
\hline BL & $=$ & American brook lamprey & Lampetra sp. \\
\hline $\mathrm{BW}$ & $=$ & Broad whitefish & :Coregonus nasus \\
\hline $\mathrm{CA}$ & $=$ & Arctic cisco & : Coregonus autumnalis \\
\hline $\mathrm{CD}$ & $=$ & Sculpin & Family Cottidae \\
\hline CI & $=$ & Cisco & Coregonus sp. \\
\hline $\mathrm{CN}$ & $=$ & Slimy sculpin & :Cottus cognatus \\
\hline $\mathrm{CS}$ & $=$ & Least cisco & :Coregonus sardinella \\
\hline $\mathrm{CT}$ & $=$ & Cutthroat trout & Orcorhynchus clarkii \\
\hline DS & $=$ & Dog (Chum) salmon & :Oncorhynchus keta \\
\hline DV & $=$ & Dolly varden & Salvelinus malma \\
\hline GR & $=$ & Grayling & :Thymallus arcticus \\
\hline $\mathrm{HO}$ & $=$ & Pond Smelt & Hypomesus olidus \\
\hline HW & $=$ & Humpback whitefish & Coregonus pidschian \\
\hline IN & $=$ & Inconnu (Sheefish) & : Stenodus leucichthys \\
\hline $\mathrm{KO}$ & $=$ & Kokanee & : Oncorhynchus nerka \\
\hline $\mathrm{KS}$ & $=$ & King (Chinook) salmon & :Ohncorhynchus tshawytscha \\
\hline LC & $=$ & Lake chub & :Couesius plumbeus \\
\hline LS & $=$ & Longnose sucker & :Catostomus catostomus \\
\hline $\mathrm{LT}$ & $=$ & Lake trout & : Salvelinus namaycush \\
\hline LW & $=$ & Lake whitefish & :Coregomis clupeaformis \\
\hline NP & $=$ & Northern pike & : Esox lucius \\
\hline OM & $=$ & Rainbow smelt & Osmerus mordax \\
\hline PS & $=$ & Pink (Humpback) salmon & :Oncorhychus gorbuscha \\
\hline PW & $=$ & Pygmy whitefish & Prosopium coulteri \\
\hline $\mathrm{RB}$ & $=$ & Rainbow trout & :Oncorhynchus mykiss \\
\hline RS & $=$ & Red (Sockeye) salmon & : Oncorhynchus nerka \\
\hline RW & $=$ & Round whitefish & :Prosopium cylindraceum \\
\hline SB & $=$ & Stickleback & :Family Gasterosteidae \\
\hline S9 & $=$ & Ninespine stickleback & : Pungitius pungitius \\
\hline SH & $=$ & Steelhead trout & : Oncorhynchus mykiss \\
\hline SK & $=$ & Sucker & :Family Catostomidae \\
\hline SS & $=$ & Coho (Silver) salmon & :Oncorhynchus kisutch \\
\hline TP & $\begin{array}{l}= \\
=\end{array}$ & Trout - Perch & Percopsis omiscomaycus \\
\hline
\end{tabular}





\begin{tabular}{|c|c|c|c|c|c|c|c|c|}
\hline USDOI & BLM PMO OPEN FILE R & OORT - TA & & FISH STREAMS $07 / 31 / 03$ & PAGE 00 & $\mathrm{OF} 00$ & & \\
\hline MP & STREAM NAME(s) & FISH & A & JaFeMrApMaJuJlAuSeOcNoDe & FIELD & : MER & REFER & : \\
\hline :A/S & : Comments & SPECIES & $: \mathrm{D}$ & $\begin{array}{l}: \text { PERIOD OF SENSITIVITY } \\
:\end{array}$ & $\begin{array}{l}: \text { STATION } \\
: \text { G-5 }\end{array}$ & $\begin{array}{l}: T \\
: R\end{array}$ & & \\
\hline & & : & $\mathrm{D}$ & & & SEC & & \\
\hline 4.12 & (Edge) Lakes & $?$ & & & 21736 & $\mathrm{U}$ & $\mathrm{ABE}$ & \\
\hline & & & & & 21796 & $10 \mathrm{~N}$ & & \\
\hline 1.37 & TAPS A/G; Causeway & & & & $1550+00$ & $14 \mathrm{E}$ & & \\
\hline & & & & & $1541+70$ & 20 & & \\
\hline 277.14 & PROSPECT CREEK & $\mathrm{CN} ; \mathrm{GR} ; \mathrm{KS}$ & $\mathrm{Y}$ & СССССССССССССССССС & 1463150 & $\mathrm{~F}$ & $\mathrm{AB}$ & \\
\hline & & $\mathrm{LS} ; \mathrm{NP} ; \mathrm{RW}$ & $\mathrm{E}$ & & 1463408 & $22 n$ & FG & \\
\hline 91 & TAPSA/G;BLOCKPOINT & & $\mathrm{S}$ & & $1590++00$ & $14 w$ & & \\
\hline & & & & & & 31 & & \\
\hline * 790,9 & (Grey Stream) & DV; SS & & CCCCSSSSSSSSjlauCCCCC & 4176212 & $\mathrm{C}$ & $\mathrm{AB} \quad \mathrm{E}$ & \\
\hline 2 & TAPS B/G; CMP & & & & & $05 \mathrm{~W}$ & & \\
\hline & & & & & $506+06$ & 28 & & \\
\hline
\end{tabular}

$\mathrm{MP}=$ The distance in miles from Pump Station 1; * Prefix denotes extrapolated mileage not field checked.

AS = Alyeska Pipeline Service Company (G-100 as-builts) alignment sheet number.

STREAM NAME = Adapted from Johnson and Rockwell, 1981. For example: YUKON RIVER denotes a name recognized by the U.S. Geological Survey; (Small or Jackie's CK) denotes a non-USGS recognized popular name; [Snowpad CK denotes a new name used in this list.

COMMENTS = TAPS A/G denotes above-ground pipe mode; TAPS B/G denotes below-ground mode; CMP is a corrugated-metal-pipe or culvert; LWC is a low water crossing; BLOCKPOINT is a physical barrier to vehicle passage; CAUSEWAY, BRIDGE, and PARALLEL are self-explanatory.

FISH SPECIES = see Page ii of iv for explanation of codes.

$\mathrm{ADAD}=$ "YES" denotes anadromous fish stream designated by Alaska Dept. Fish and Game.

PERIOD OF SENSITIVITY = C denotes Critical period of fish usage; $\mathrm{S}$ denotes Sensitive period of fish usage. NOTE: UNDERLINING of a stream's period of sensitivity denotes the recommended sensitivity period if fish return in the future.

FIELD STATION = Distance in feet from Pump Station No 1 as estimated in field; * Prefix denotes an extrapolated stationing not field checked.

G-5 = Obtained from selected references (see Page iii of iv) and refers to construction drawings.

MER = Meridian $-U$ is Umiat; $F$ is Fairbanks; $C$ is Copper River.

$\mathrm{T}=$ Tier or Township; $\mathrm{R}=$ Range Sec. $=$ Section .

REFERENCE $=$ see page iii of iv. 
USDOI BLM PMO OPEN FILE REPORT - TAPS FISH STREAMS

\begin{tabular}{|c|c|c|c|c|c|c|}
\hline MP & STREAM NAME(s) & $\begin{array}{l}\text { FISH } \\
\text { SPECIES }\end{array}$ & $\begin{array}{l}\text { A : JaFeMrApMaJuJIAuSeOcNoDe } \\
\text { D : PERIOD OF SENSITIVITY }\end{array}$ & $\begin{array}{l}\text { FIELD } \\
\text { STATION }\end{array}$ & $\begin{array}{l}\text { MER } \\
T\end{array}$ & $\begin{array}{l}\text { REFER } \\
\text {--ENCE }\end{array}$ \\
\hline $\mathrm{A} / \mathrm{S}$ & Comments & & & G-5 & $\begin{array}{l}: \mathrm{R} \\
: \mathrm{SEC}\end{array}$ & \\
\hline 1.37 & $\begin{array}{l}\text { (Edge) Lakes } \\
\text { TAPS A/G; Causeway }\end{array}$ & $?$ & 4 & $\begin{array}{c}20842 \\
21314 \\
1550+00 \\
1541+70\end{array}$ & $\begin{array}{l}\mathrm{U} \\
10 \mathrm{~N} \\
14 \mathrm{E} \\
20\end{array}$ & $\begin{array}{l}\mathrm{AB} \\
\mathrm{F}\end{array}$ \\
\hline $\begin{array}{r}4.12 \\
137\end{array}$ & $\begin{array}{l}\text { (Edge) Lakes } \\
\text { TAPS A/G; LWC }\end{array}$ & & & $\begin{array}{c}21736 \\
21796 \\
1550+00 \\
1541+70\end{array}$ & $\begin{array}{l}\mathrm{U} \\
10 \mathrm{~N} \\
14 \mathrm{E} \\
20\end{array}$ & $\begin{array}{l}\mathrm{AB} \\
\mathrm{F}\end{array}$ \\
\hline $\begin{array}{r}4.12 \\
137\end{array}$ & $\begin{array}{l}\text { (Edge) Lakes } \\
\text { TAPS A/G; Causeway }\end{array}$ & & & $\begin{array}{c}21496 \\
21976 \\
1550+00 \\
1541+70 \\
\end{array}$ & $\begin{array}{l}\mathrm{U} \\
10 \mathrm{~N} \\
14 \mathrm{E} \\
20 \\
\end{array}$ & $\begin{array}{l}\overline{\mathrm{AB}} \\
\mathrm{F}\end{array}$ \\
\hline $\begin{array}{r}5.12 \\
137\end{array}$ & $\begin{array}{l}\text { (Grayling Gulch) } \\
\text { TAPS A/G; BLOCKPOINT }\end{array}$ & ? & & $\begin{array}{c}26939 \\
27159 \\
1478+52\end{array}$ & $\begin{array}{l}\mathrm{U} \\
10 \mathrm{~N} \\
14 \mathrm{E} \\
29\end{array}$ & $\begin{array}{l}\mathrm{AB} \\
\mathrm{F}\end{array}$ \\
\hline $\begin{array}{r}11.10 \\
136\end{array}$ & $\begin{array}{l}\text { Unnamed Lake } \\
\text { TAPS A/G; LWC }\end{array}$ & S9 & jafemrapSSSSSSSSSeocnode & $\begin{array}{l}58498 \\
58558\end{array}$ & $\begin{array}{l}\mathrm{U} \\
19 \mathrm{~N} \\
14 \mathrm{E} \\
28 \\
\end{array}$ & $\mathrm{~J}$ \\
\hline $\begin{array}{r}17.98 \\
135\end{array}$ & $\begin{array}{l}\text { (Low Life CK) } \\
\text { TAPS B/G; LWC }\end{array}$ & S9 & jafemrapSSSSSSSSSeocnode & $\begin{array}{r}94930 \\
825+00\end{array}$ & $\begin{array}{l}\mathrm{U} \\
08 \mathrm{~N} \\
14 \mathrm{E} \\
28\end{array}$ & $\overline{\mathrm{E}}$ \\
\hline $\begin{array}{r}20.55 \\
22.41 \\
134\end{array}$ & $\begin{array}{l}\text { SAGAVANIRKTOK RIVER } \\
\text { SIDE CHANNELS and } \\
\text { FLOODPLAIN } \\
\text { TAPS B/G; LWCs }\end{array}$ & $\begin{array}{l}\mathrm{AC} ? \mathrm{CN} \\
\mathrm{GR}, \mathrm{RW}\end{array}$ & $\begin{array}{l}\text { Y jafemrapCCCCSSauseocnode } \\
\text { E } \\
\text { S Sag River mainstem system and all } \\
\text { side channels are specified as being } \\
\text { important for the spawning, rearing or } \\
\text { migration of anadromous fish. }\end{array}$ & $\begin{array}{r}108528 \\
118300 \\
5479+00 \\
5396+10\end{array}$ & $\begin{array}{l}U \\
17 \mathrm{~N} \\
14 \mathrm{E} \\
8,17 \\
18 \& 19\end{array}$ & $\begin{array}{ll}\mathrm{AB} & \mathrm{E} \\
\mathrm{F} & \end{array}$ \\
\hline 134 & $\begin{array}{l}\text { Pond } \\
\text { TAPS B/G;PARALLEL ONLY } \\
\text { TAPS DOES NOT CROSS }\end{array}$ & GR & \begin{tabular}{|l:l} 
& jafemrapCCCCSauseocnode
\end{tabular} & $\begin{array}{l}5388+33 \\
5383+55\end{array}$ & $\begin{array}{l}\mathrm{U} \\
17 \mathrm{~N} \\
14 \mathrm{E} \\
19 \\
\end{array}$ & B \\
\hline 134 & $\begin{array}{l}\text { Pond } \\
\text { TAPS B/G;PARALLEL ONLY } \\
\text { TAPS DOES NOT CROSS }\end{array}$ & GR & jafemrapCCCCSSauseocnode & $\begin{array}{l}5374+75 \\
5370+50\end{array}$ & $\begin{array}{l}\mathrm{U} \\
17 \mathrm{~N} \\
14 \mathrm{E} \\
19\end{array}$ & B \\
\hline $\begin{array}{r}24.03 \\
134\end{array}$ & $\begin{array}{l}\text { SAGAVANIRKTOK RIVER } \\
\text { SIDE CHANNEL } \\
\text { TAPS B/G; LWC }\end{array}$ & AC?GR & jafemrapCCCCSSauseocnode & $\begin{array}{r}126900 \\
5296+83\end{array}$ & $\begin{array}{ll}\mathrm{U} \\
07 \mathrm{~N} \\
14 \mathrm{E} \\
30\end{array}$ & $\begin{array}{l}\mathrm{AB} \\
\mathrm{F}\end{array}$ \\
\hline 24.91 & $\begin{array}{l}\text { SAGAVANIRKTOK RIVER } \\
\text { SIDE CHANNEL } \\
\text { TAPS B/G; LWC }\end{array}$ & AC?GR & jafemrapCCCCSSauseocnode & $\begin{array}{r}131525 \\
5251+61\end{array}$ & $\begin{array}{l}\mathrm{U} \\
07 \mathrm{~N} \\
14 \mathrm{E} \\
31\end{array}$ & $\begin{array}{ll}\mathrm{AB} & \mathrm{E} \\
\mathrm{F} & \end{array}$ \\
\hline 25.10 & $\begin{array}{l}\text { SAGAVANIRKTOK RIVER } \\
\text { SIDE CHANNEL } \\
\text { TAPS B/G; LWC }\end{array}$ & AC?GR & jafemrapCCCCSSauseocnode & $\begin{array}{r}132525 \\
5241+61\end{array}$ & $\begin{array}{l}U \\
07 \mathrm{~N} \\
14 \mathrm{E} \\
31 \\
\end{array}$ & F \\
\hline 25.15 & $\begin{array}{l}\text { SAGAVANIRKTOK RIVER } \\
\text { SIDE CHANNEL } \\
\text { TAPS B/G; LWC }\end{array}$ & AC?GR & jafemrapCCCCSSauseocnode & $\begin{array}{r}132810 \\
5238+76\end{array}$ & $\begin{array}{l}U \\
07 \mathrm{~N} \\
14 \mathrm{E} \\
31\end{array}$ & $\mathrm{~F}$ \\
\hline $\begin{array}{r}25.56 \\
133\end{array}$ & $\begin{array}{l}\text { SAGAVANIRKTOK RIVER } \\
\text { SIDE CHANNEL } \\
\text { TAPS B/G; LWC }\end{array}$ & AC?GR & jafemrapCCCCSSauseocnode & $\begin{array}{r}134950 \\
5210+93\end{array}$ & $\begin{array}{l}\mathrm{U} \\
06 \mathrm{~N} \\
14 \mathrm{E} \\
06\end{array}$ & $\overline{\mathrm{AB}}$ \\
\hline
\end{tabular}


USDOI BLM PMO OPEN FILE REPORT - TAPS FISH STREAMS 07/30/03 PAGE 2 of 41

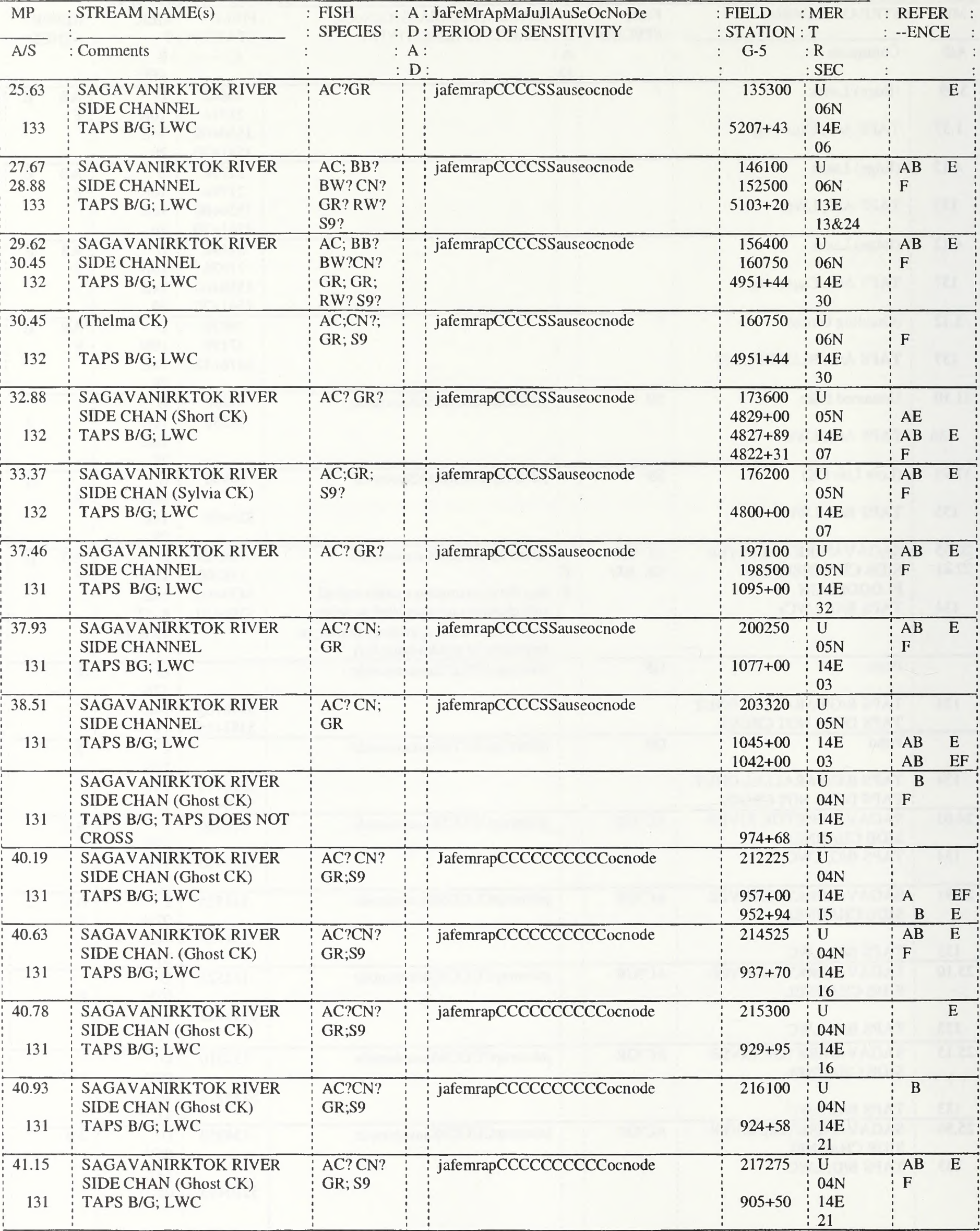


USDOI BLM PMO OPEN FILE REPORT - TAPS FISH STREAMS

\begin{tabular}{|c|c|c|c|c|c|c|c|}
\hline MP & : STREAM NAME(s) & $\begin{array}{l}\text { FISH } \\
\text { SPECIES }\end{array}$ & $\begin{array}{l}\text { A : JaFeMrApMaJuJlAuSeOcNoDe } \\
\text { D : PERIOD OF SENSITIVITY }\end{array}$ & $\begin{array}{l}\text { : FIELD } \\
: \text { STATION }\end{array}$ & $\begin{array}{l}: \text { MER } \\
: T\end{array}$ & $\begin{array}{l}\text { REFE } \\
- \text { ENC }\end{array}$ & \\
\hline $\mathrm{A} / \mathrm{S}$ & Comments & & $\begin{array}{l}\text { A : } \\
\text { D : }\end{array}$ & : G-5 & $\begin{array}{l}: \mathbf{R} \\
: \text { SEC }\end{array}$ & & \\
\hline $\begin{array}{r}41.39 \\
131\end{array}$ & $\begin{array}{l}\text { SAGAVANIRKTOK RIVER } \\
\text { TAPS B/G; LWC }\end{array}$ & $\begin{array}{l}\mathrm{AC} ? \mathrm{CN} ? \\
\mathrm{GR} ; \mathrm{S} 9\end{array}$ & jafemrapCCCCCCCCCCocnode & $\begin{array}{l}218525 \\
893+50\end{array}$ & $\begin{array}{l}\mathrm{U} \\
04 \mathrm{~N} \\
\mathrm{I} 4 \mathrm{E} \\
2 \mathrm{I} \\
\end{array}$ & $\begin{array}{l}\mathrm{AB} \\
\mathrm{F}\end{array}$ & $\mathrm{E}$ \\
\hline $\begin{array}{r}41.77 \\
131\end{array}$ & $\begin{array}{l}\text { SAGAVANIRKTOK RIVER } \\
\text { SIDE CHAN (Ghost CK) } \\
\text { TAPS B/G; LWC }\end{array}$ & $\begin{array}{l}\mathrm{AC} ? \mathrm{CN} ? \\
\text { GR; S9 }\end{array}$ & jafemrapCCCCCCCCCCocnode & $\begin{array}{l}220525 \\
872+00\end{array}$ & $\begin{array}{l}\mathrm{U} \\
04 \mathrm{~N} \\
14 \mathrm{E} \\
2 \mathrm{I}\end{array}$ & $\begin{array}{l}\mathrm{AB} \\
\mathrm{F}\end{array}$ & $\mathrm{E}$ \\
\hline $\begin{array}{r}42.12 \\
130\end{array}$ & $\begin{array}{l}\text { SAGAVAN1RKTOK RIVER } \\
\text { S1DE CHAN (Ghost CK) } \\
\text { TAPS B/G; LWC }\end{array}$ & $\begin{array}{l}\mathrm{AC} ? \mathrm{CN} ? \\
\mathrm{GR} ; \mathrm{S} 9\end{array}$ & : jafemrapCCCCCCCCCCocnode & $\begin{array}{l}222500 \\
853+25\end{array}$ & $\begin{array}{l}\mathrm{U} \\
04 \mathrm{~N} \\
\mathrm{I} 4 \mathrm{E} \\
27\end{array}$ & B & $\mathrm{E}$ \\
\hline $\begin{array}{r}42.25 \\
130\end{array}$ & $\begin{array}{l}\text { SAGAVANIRKTOK RIVER } \\
\text { SIDE CHAN (Ghost CK) } \\
\text { TAPS B/G; LWC }\end{array}$ & $\begin{array}{l}\mathrm{AC} ? \mathrm{CN} ? \\
\text { GR; S9 }\end{array}$ & jafemrapCCCCCCCCCCocnode & $\begin{array}{l}223100 \\
846+16\end{array}$ & $\begin{array}{l}\mathrm{U} \\
04 \mathrm{~N} \\
\mathrm{I} 4 \mathrm{E} \\
27\end{array}$ & $\mathrm{~B}$ & \\
\hline $\begin{array}{r}42.26 \\
130\end{array}$ & $\begin{array}{l}\text { SAGAVANIRKTOK RIVER } \\
\text { SIDE CHAN (Ghost CK) } \\
\text { TAPS B/G; LWC }\end{array}$ & $\begin{array}{l}\text { AC? CN? } \\
\text { GR; S9 }\end{array}$ & jafemrapCCCCCCCCCCoenode & $\begin{array}{l}223150 \\
843+08\end{array}$ & $\begin{array}{l}\mathrm{U} \\
04 \mathrm{~N} \\
\mathrm{I} 4 \mathrm{E} \\
27 \\
\end{array}$ & B & $\mathrm{E}$ \\
\hline $\begin{array}{r}42.52 \\
130\end{array}$ & $\begin{array}{l}\text { SAGAVANIRKTOK RIVER } \\
\text { SIDE CHAN (Ghost CK) } \\
\text { TAPS B/G; LWC }\end{array}$ & $\begin{array}{l}\mathrm{AC} ? \mathrm{CN} ? \\
\mathrm{GR} ; \mathrm{S} 9\end{array}$ & jafemrapCCCCCCCCCCocnode & $\begin{array}{l}224525 \\
831+30\end{array}$ & $\begin{array}{l}\mathrm{U} \\
04 \mathrm{~N} \\
14 \mathrm{E} \\
27 \\
\end{array}$ & $\begin{array}{l}\mathrm{AB} \\
\mathrm{F}\end{array}$ & \\
\hline $\begin{array}{r}42.63 \\
130\end{array}$ & $\begin{array}{l}\text { SAGAVANIRKTOK RIVER } \\
\text { SIDE CHAN (Ghost CK) } \\
\text { TAPS B/G; LWC }\end{array}$ & $\begin{array}{l}\mathrm{AC} ? \mathrm{CN} ? \\
\mathrm{GR} ; \mathrm{S} 9\end{array}$ & jafemrapCCCCCCCCCCocnode & $\begin{array}{l}225075 \\
826+50\end{array}$ & $\begin{array}{l}\text { U04N } \\
\text { I4E } \\
27\end{array}$ & $\begin{array}{l}\mathrm{AB} \\
\mathrm{F}\end{array}$ & \\
\hline $\begin{array}{r}43.03 \\
130\end{array}$ & $\begin{array}{l}\text { SAGAVANIRKTOK RIVER } \\
\text { SIDE CHAN (Ghost CK) } \\
\text { TAPS B/G; LWC }\end{array}$ & $\begin{array}{l}\mathrm{AC} ? \mathrm{CN} ? \\
\mathrm{GR} ; \mathrm{S} 9\end{array}$ & jafemrapCCCCCCCCCCocnode & $\begin{array}{l}227200 \\
804+68\end{array}$ & $\begin{array}{l}\mathrm{U} \\
04 \mathrm{~N} \\
14 \mathrm{E} \\
34 \\
\end{array}$ & $\mathrm{~F}^{\mathrm{B}}$ & \\
\hline $\begin{array}{r}43.71 \\
130\end{array}$ & $\begin{array}{l}\text { SAGAVANIRKTOK RIVER } \\
\text { SIDE CHAN (Ghost CK) } \\
\text { TAPS B/G; LWC }\end{array}$ & $\begin{array}{l}\mathrm{AC} ? \mathrm{CN} ? \\
\mathrm{GR} ; \mathrm{S} 9\end{array}$ & jafemrapCCCCCCCCCCocnode & $\begin{array}{l}230800 \\
768+86\end{array}$ & $\begin{array}{l}\mathrm{U} \\
04 \mathrm{~N} \\
14 \mathrm{E} \\
34 \\
\end{array}$ & B & \\
\hline $\begin{array}{r}43.95 \\
130\end{array}$ & $\begin{array}{l}\text { SAGAVAN1RKTOK RIVER } \\
\text { SIDE CHAN (Ghost CK) } \\
\text { TAPS B/G; LWC }\end{array}$ & $\begin{array}{l}\mathrm{AC} ? \mathrm{CN} ? \\
\mathrm{GR} ; \mathrm{S} 9\end{array}$ & jafemrapCCCCCCCCCCoenode & $\begin{array}{l}232075 \\
756+49\end{array}$ & $\begin{array}{l}U \\
04 \mathrm{~N} \\
14 \mathrm{E} \\
34 \\
\end{array}$ & B & \\
\hline $\begin{array}{r}44.34 \\
130\end{array}$ & $\begin{array}{l}\text { SAGAVANIRKTOK RIVER } \\
\text { SIDE CHAN (Ghost CK) } \\
\text { TAPS B/G; LWC }\end{array}$ & $\begin{array}{l}\text { AC? CN? } \\
\text { GR; S9 }\end{array}$ & jafemrapCCCCCCCCCCocnode & $\begin{array}{l}234100 \\
736+81\end{array}$ & $\begin{array}{l}\mathrm{U} \\
03 \mathrm{~N} \\
14 \mathrm{E} \\
03 \\
\end{array}$ & B & \\
\hline $\begin{array}{l}44.50 \\
130\end{array}$ & $\begin{array}{l}\text { SAGAVANIRKTOK RIVER } \\
\text { SIDE CHAN (Ghost CK) } \\
\text { TAPS B/G; LWC }\end{array}$ & $\begin{array}{l}\text { AC? CN? } \\
\text { GR; S9 }\end{array}$ & jafemrapCCCCCCCCCCocnode & $\begin{array}{l}234950 \\
728+68\end{array}$ & $\begin{array}{l}\mathrm{U} \\
03 \mathrm{~N} \\
14 \mathrm{E} \\
03 \\
\end{array}$ & B & \\
\hline $\begin{array}{c}47.50 \\
48.98 \\
130 \\
129\end{array}$ & $\begin{array}{l}\text { SAGAVAN1RKTOK RIVER } \\
\text { SIDE CHANNELS AND } \\
\text { FLOODPLAIN (Extension } \\
\text { CK)) } \\
\text { TAPS B/G; LWC }\end{array}$ & $\begin{array}{l}\text { AC? CN? } \\
\text { GR; S9 }\end{array}$ & jafemrapCCCCSSSCCCocnode & $\begin{array}{l}251000 \\
258600 \\
557+50 \\
539+10 \\
531+70 \\
531+00 \\
525+10 \\
500+25 \\
500+00 \\
492+35 \\
491+40 \\
\end{array}$ & $\begin{array}{l}\mathrm{U} \\
03 \mathrm{~N} \\
14 \mathrm{E} \\
23 \\
\& \\
26\end{array}$ & \begin{tabular}{|l}
$\mathrm{A}$ \\
${ }^{\mathrm{B}}$ \\
$\mathrm{B}$ \\
$\mathrm{A}$ \\
$\mathrm{A}$ \\
$\mathrm{A}$ \\
${ }^{\mathrm{B}}$ \\
$\mathrm{A}^{\mathrm{B}}$
\end{tabular} & 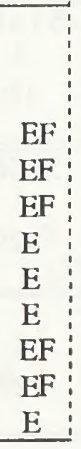 \\
\hline
\end{tabular}


USDOI BLM PMO OPEN FILE REPORT - TAPS FISH STREAMS

\begin{tabular}{|c|c|c|c|c|c|c|}
\hline MP & : STREAM NAME(s) & $\begin{array}{l}\text { FISH } \\
\text { SPECIES }\end{array}$ & $\begin{array}{l}\text { A : JaFeMrApMaJuJIAuSeOcNoDe } \\
\text { D : PERIOD OF SENSITIVITY }\end{array}$ & $\begin{array}{l}\text { : FIELD } \\
\text { : STATION }\end{array}$ & $\begin{array}{l}\text { MER } \\
T\end{array}$ & $\begin{array}{l}\text { : REFER } \\
: \text {--ENCE }\end{array}$ \\
\hline $\mathrm{A} / \mathrm{S}$ & : Comments & & $\begin{array}{l}\text { A : } \\
\text { D : }\end{array}$ & G-5 & $\begin{array}{l}\text { R } \\
\text { SEC }\end{array}$ & \\
\hline $\begin{array}{r}49.99 \\
129\end{array}$ & $\begin{array}{l}\text { SAGAVANIRKTOK RIVER } \\
\text { SIDE CHANNEL } \\
\text { TAPS B/G; LWC }\end{array}$ & $\begin{array}{l}\mathrm{AC} ? \mathrm{GR} \\
\text { S9 }\end{array}$ & jafemrapCCCCSSSCCeocnode & $\begin{array}{l}263950 \\
437+00\end{array}$ & $\begin{array}{l}\mathrm{U} \\
03 \mathrm{~N} \\
14 \mathrm{E} \\
35\end{array}$ & $\mathrm{~F}^{\mathrm{B} \quad \mathrm{E}}$ \\
\hline $\begin{array}{r}50.11 \\
129\end{array}$ & $\begin{array}{l}\text { SAGAVANIRKTOK RIVER } \\
\text { SIDE CHANNEL } \\
\text { TAPS B/G; LWC }\end{array}$ & $\begin{array}{l}\mathrm{AC} ? \mathrm{GR} \\
\text { S9 }\end{array}$ & jafemrapCCCCSSSCCeocnode & $\begin{array}{l}264600 \\
430+00\end{array}$ & $\begin{array}{l}\mathrm{U} \\
03 \mathrm{~N} \\
14 \mathrm{E} \\
35 \\
\end{array}$ & $\begin{array}{lll}\text { A B } & \text { E } \\
\text { F } & \\
& & \\
\end{array}$ \\
\hline 129 & $\begin{array}{l}\text { SAGAVANIRKTOK RIVER } \\
\text { SIDE CHANNEL } \\
\text { TAPS B/G; NO TAPS } \\
\text { CROSSING }\end{array}$ & & & 414.20 & & \\
\hline $\begin{array}{r}50.47 \\
129\end{array}$ & $\begin{array}{l}\text { SAGAVANIRKTOK RIVER } \\
\text { SIDE CHANNEL } \\
\text { TAPS B/G; LWC }\end{array}$ & $\begin{array}{l}\mathrm{AC} ? \mathrm{GR} \\
\text { S9 }\end{array}$ & jafemrapCCCCSSSCCeocnode & $\begin{array}{l}266500 \\
412+57\end{array}$ & $\begin{array}{l}\mathrm{U} \\
02 \mathrm{~N} \\
\mathrm{I} 4 \mathrm{E} \\
02\end{array}$ & $\begin{array}{cc}\mathrm{B} \quad \mathrm{E} \\
\mathrm{F}\end{array}$ \\
\hline $\begin{array}{r}50.5 \\
129\end{array}$ & $\begin{array}{l}\text { SAGAVANIRKTOK RIVER } \\
\text { SIDE CHANNEL } \\
\text { TAPS B/G; PARALLEL ONLY }\end{array}$ & $\begin{array}{l}\text { AC? GR; } \\
\text { S9 }\end{array}$ & jafemrapCCCCSSSCCeocnode & $\begin{array}{l}\text { NO TAPS } \\
\text { XING } \\
410+53\end{array}$ & $\begin{array}{l}\mathrm{U} \\
02 \mathrm{~N} \\
14 \mathrm{E} \\
02\end{array}$ & $\underset{F}{B} \quad \mathrm{E}$ \\
\hline $\begin{array}{r}50.76 \\
129\end{array}$ & $\begin{array}{l}\text { SAGAVANIRKTOK RIVER } \\
\text { SIDE CHAN. (Wood CK) } \\
\text { TAPS B/G; LWC }\end{array}$ & $\begin{array}{l}\text { AC? CN? } \\
\text { GR; S9 }\end{array}$ & jafemrapCCCCSSCCCCocnode & $\begin{array}{l}268000 \\
396+00 \\
395+41 \\
\end{array}$ & $\begin{array}{l}\mathrm{U} \\
02 \mathrm{~N} \\
14 \mathrm{E} \\
01\end{array}$ & $\begin{array}{ll}\text { A } & \text { E G } \\
\text { B } & \text { EFG }\end{array}$ \\
\hline $\begin{array}{r}51.46 \\
129\end{array}$ & $\begin{array}{l}\text { SAGAVANIRKTOK RIVER } \\
\text { SIDE CHAN. (Wood CK) } \\
\text { TAPS B/G; LWC }\end{array}$ & $\begin{array}{l}\text { AC? CN? } \\
\text { GR; S9 }\end{array}$ & jafemrapCCCCSSCCCCocnode & $\begin{array}{l}271700 \\
360+60\end{array}$ & $\begin{array}{l}\mathrm{U} \\
02 \mathrm{~N} \\
\mathrm{I} 4 \mathrm{E} \\
\mathrm{I} 2\end{array}$ & $\begin{array}{cc}\text { B } & \text { E } \\
\text { G } & \end{array}$ \\
\hline $\begin{array}{r}51.55 \\
\mathrm{I} 29\end{array}$ & $\begin{array}{l}\text { SAGAVANIRKTOK RIVER } \\
\text { SIDE CHAN. (Wood CK) } \\
\text { TAPS B/G; LWC }\end{array}$ & $\begin{array}{l}\text { AC? CN? } \\
\text { GR; S9 }\end{array}$ & jafemrapCCCCSSCCCCocnode & $\begin{array}{l}272200 \\
355+07\end{array}$ & $\begin{array}{l}\mathrm{U} \\
02 \mathrm{~N} \\
\mathrm{I} \mathrm{E} \\
12\end{array}$ & $\begin{array}{r}\mathrm{B} \\
\text { FG }\end{array}$ \\
\hline $\begin{array}{r}52.14 \\
129\end{array}$ & $\begin{array}{l}\text { SAGAVANIRKTOK RIVER } \\
\text { SIDE CHAN. (Wood CK) } \\
\text { TAPS B/G; LWC }\end{array}$ & $\begin{array}{l}\mathrm{AC} ? \mathrm{CN} ? \\
\mathrm{GR} ; \mathrm{S} 9\end{array}$ & jafemrapCCCCSSCCCCocnode & $\begin{array}{l}275300 \\
322+25\end{array}$ & $\begin{array}{l}\mathrm{U} \\
02 \mathrm{~N} \\
\mathrm{I} 4 \mathrm{E} \\
12\end{array}$ & $\begin{array}{l}\mathrm{AB} \\
\mathrm{FG}\end{array}$ \\
\hline $\begin{array}{l}52.97 \\
129\end{array}$ & $\begin{array}{l}\text { SAGAVANIRKTOK RIVER } \\
\text { SIDE CHAN. (Wood CK) } \\
\text { TAPS B/G; LWC }\end{array}$ & $\begin{array}{l}\mathrm{AC} ? \mathrm{CN} ? \\
\mathrm{GR} ; \mathrm{S} 9\end{array}$ & jafemrapCCCCSSCCCCocnode & $\begin{array}{r}279700 \\
28 I+50\end{array}$ & $\begin{array}{l}\mathrm{U} \\
02 \mathrm{~N} \\
14 \mathrm{E} \\
13\end{array}$ & $\begin{array}{r}\text { B } \\
\text { FG }\end{array}$ \\
\hline $\begin{array}{r}53.11 \\
129\end{array}$ & $\begin{array}{l}\text { SAGAVANIRKTOK RIVER } \\
\text { SIDE CHAN. (Wood CK) } \\
\text { TAPS B/G; LWC }\end{array}$ & $\begin{array}{l}\text { AC? CN? } \\
\text { GR; S9 }\end{array}$ & jafemrapCCCCSSCCCCocnode & $\begin{array}{l}280400 \\
266+00\end{array}$ & $\begin{array}{l}\mathrm{U} \\
02 \mathrm{~N} \\
14 \mathrm{E} \\
13\end{array}$ & $\mathrm{~A}$ \\
\hline $\begin{array}{r}53.33 \\
129\end{array}$ & $\begin{array}{l}\text { SAGAVANIRKTOK RIVER } \\
\text { SIDE CHAN. (Wood CK) } \\
\text { TAPS B/G; LWC }\end{array}$ & $\begin{array}{l}\mathrm{AC} ? \mathrm{CN} ? \\
\mathrm{GR} ; \mathrm{S} 9\end{array}$ & jafemrapCCCCSSCCCCocnode & $\begin{array}{l}28 I 600 \\
265+76\end{array}$ & $\begin{array}{l}\mathrm{U} \\
02 \mathrm{~N} \\
\mathrm{I4E} \\
13\end{array}$ & $\begin{array}{c}\text { B } \\
\text { FG }\end{array}$ \\
\hline $\begin{array}{r}53.41 \\
129\end{array}$ & $\begin{array}{l}\text { SAGAVANIRKTOK RIVER } \\
\text { SIDE CHAN. (Wood CK) } \\
\text { TAPS B/G; LWC }\end{array}$ & $\begin{array}{l}\mathrm{AC} ? \mathrm{CN} ? \\
\mathrm{GR} ; \mathrm{S} 9\end{array}$ & jafemrapCCCCSSCCCCocnode & $\begin{array}{l}282000 \\
258+6 I\end{array}$ & $\begin{array}{l}\mathrm{U} \\
02 \mathrm{~N} \\
14 \mathrm{E} \\
24\end{array}$ & $\begin{array}{r}\text { B } \\
\text { FG }\end{array}$ \\
\hline $\begin{array}{r}53.56 \\
129\end{array}$ & $\begin{array}{l}\text { SAGAVANIRKTOK RIVER } \\
\text { SIDE CHAN. (Wood CK) } \\
\text { TAPS B/G; LWC }\end{array}$ & $\begin{array}{l}\mathrm{AC} ? \mathrm{CN} ? \\
\mathrm{GR} ; \mathrm{S} 9\end{array}$ & jafemrapCCCCSSCCCCocnode & $\begin{array}{l}282800 \\
246+28\end{array}$ & $\begin{array}{l}\mathrm{U} \\
02 \mathrm{~N} \\
\mathrm{I} \mathrm{E} \\
24\end{array}$ & \begin{tabular}{|c|} 
B \\
FG
\end{tabular} \\
\hline 129 & $\begin{array}{l}\text { SAGAVANIRKTOK RIVER } \\
\text { SIDE CHANEL } \\
\text { TAPS B/G; TAPS DOES NOT } \\
\text { CROSS }\end{array}$ & & & $\begin{array}{l}\text { NO TAPS } \\
\text { XING } \\
242+80\end{array}$ & & \begin{tabular}{|cc} 
B & E \\
FG & \\
\end{tabular} \\
\hline
\end{tabular}


USDOI BLM PMO OPEN FILE REPORT - TAPS FISH STREAMS

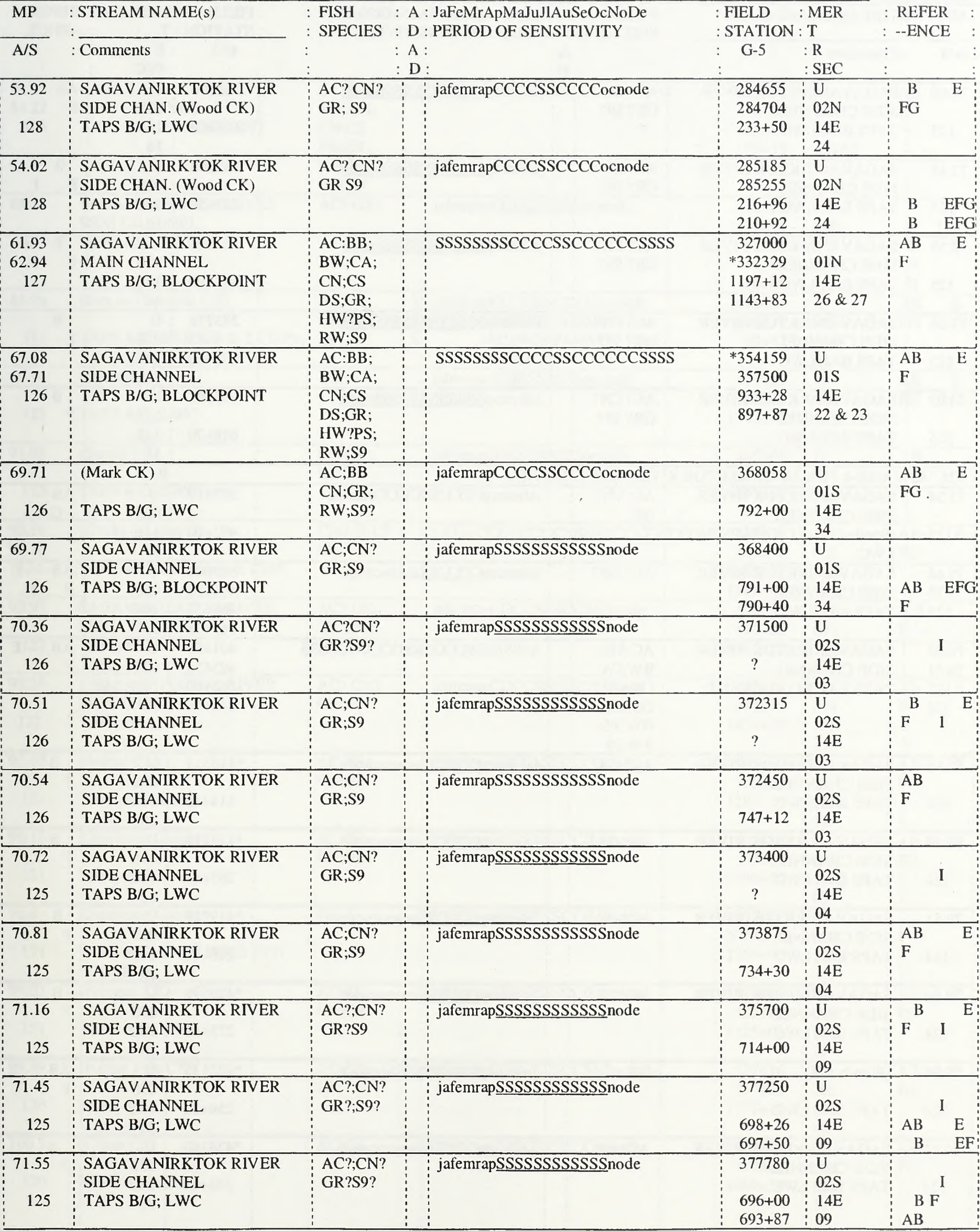


USDOI BLM PMO OPEN FILE REPORT - TAPS FISH STREAMS

07/30/03 PAGE 6 of 41

\begin{tabular}{|c|c|c|c|c|c|c|}
\hline $\mathrm{MP}$ & : STREAM NAME(s) & $\begin{array}{l}\text { FISH } \\
\text { SPECIES }\end{array}$ & $\begin{array}{l}\text { A : JaFeMrApMaJuJIAuSeOcNoDe } \\
\text { D : PERIOD OF SENSITIVITY }\end{array}$ & $\begin{array}{l}\text { FIELD } \\
\text { STATION }\end{array}$ & $\begin{array}{l}\text { MER } \\
T\end{array}$ & $\begin{array}{l}\text { REFER } \\
\text {--ENCE }\end{array}$ \\
\hline $\mathrm{A} / \mathrm{S}$ & Comments & & $\begin{array}{l}\text { A : } \\
\text { D : }\end{array}$ & G-5 & $\begin{array}{l}: \mathrm{R} \\
: \text { SEC }\end{array}$ & \\
\hline $\begin{array}{r}72.06 \\
125\end{array}$ & $\begin{array}{l}\text { SAGAVANIRKTOK RIVER } \\
\text { SIDE CHANNEL } \\
\text { TAPS B/G; LWC }\end{array}$ & $\begin{array}{l}\text { AC? CN? } \\
\text { GR? S9? }\end{array}$ & jafemrapSSSSSSSSSSSSSnode & $\begin{array}{l}380500 \\
666+00\end{array}$ & $\begin{array}{l}\mathrm{U} \\
02 \mathrm{~S} \\
14 \mathrm{E} \\
16\end{array}$ & $\begin{array}{ll}A B & E \\
F\end{array}$ \\
\hline $\begin{array}{r}72.45 \\
125\end{array}$ & $\begin{array}{l}\text { SAGAVANIRKTOK RIVER } \\
\text { SIDE CHANNEL } \\
\text { TAPS B/G; LWC }\end{array}$ & $\begin{array}{l}\mathrm{AC} \text { ? CN? } \\
\text { GR? S9? }\end{array}$ & jafemrapSSSSSSSSSSSSnode & $\begin{array}{l}382568 \\
643+50\end{array}$ & $\begin{array}{l}U \\
02 S \\
14 E \\
16\end{array}$ & $F^{B}$ \\
\hline $\begin{array}{r}72.59 \\
125\end{array}$ & $\begin{array}{l}\text { SAGAVANIRKTOK RIVER } \\
\text { SIDE CHANNEL } \\
\text { TAPS B/G; LWC } \\
\end{array}$ & $\begin{array}{l}\text { AC? CN? } \\
\text { GR? S9? }\end{array}$ & jafemrapSSSSSSSSSSSSnode & $\begin{array}{l}383268 \\
637+00\end{array}$ & $\begin{array}{l}\mathrm{U} \\
02 \mathrm{~S} \\
14 \mathrm{E} \\
16\end{array}$ & $\mathrm{~F}^{\mathrm{B}}$ \\
\hline $\begin{array}{r}72.68 \\
125\end{array}$ & $\begin{array}{l}\text { SAGAVANIRKTOK RIVER } \\
\text { SIDE CHANNEL } \\
\text { TAPS B/G; LWC }\end{array}$ & $\begin{array}{l}\text { AC? CN? } \\
\text { GR? S9? }\end{array}$ & jafemrapSSSSSSSSSSSSnode & $\begin{array}{l}383778 \\
632+50\end{array}$ & $\begin{array}{l}\mathrm{U} \\
02 \mathrm{~S} \\
14 \mathrm{E} \\
16\end{array}$ & B \\
\hline $\begin{array}{r}73.03 \\
125\end{array}$ & $\begin{array}{l}\text { SAGAVANIRKTOK RIVER } \\
\text { SIDE CHANNEL } \\
\text { TAPS B/G; LWC }\end{array}$ & $\begin{array}{l}\text { AC? CN? } \\
\text { GR? S9? }\end{array}$ & jafemrapSSSSSSSSSSSSnode & $\begin{array}{l}385607 \\
616+70\end{array}$ & $\begin{array}{l}\mathrm{U} \\
02 \mathrm{~S} \\
14 \mathrm{E} \\
16 \\
\end{array}$ & F \\
\hline $125 \mathrm{~A}$ & LAMS-4 SAGAVANIRKTOK & 1VER SIDE & HANNEL & & $\mathrm{B}$ & \\
\hline $\begin{array}{r}75.34 \\
125\end{array}$ & $\begin{array}{l}\text { SAGAVANIRKTOK RIVER } \\
\text { SIDE CHANNEL } \\
\text { (Spoiled Mary CK )TAPS B/G; } \\
\text { LWC }\end{array}$ & $\begin{array}{l}\mathrm{AC} ; \mathrm{CN} \\
\mathrm{GR}\end{array}$ & \begin{tabular}{l|l} 
& jafemrapCCCSSCCCocnode
\end{tabular} & $\begin{array}{l}397819 \\
492+00\end{array}$ & $\begin{array}{l}\mathrm{U} \\
02 \mathrm{~S} \\
14 \mathrm{E} \\
33 \\
\end{array}$ & $\begin{array}{r}\text { AB } \\
\text { G }\end{array}$ \\
\hline $\begin{array}{r}75.68 \\
75.79 \\
125\end{array}$ & $\begin{array}{l}\text { SAGAVANIRKTOK RIVER } \\
\text { SIDE CHANNEL } \\
\text { TAPS B/G; LWC }\end{array}$ & AC? GR? & jafemrapCCCCSSauseocnode & $\begin{array}{l}399600 \\
400150 \\
489+35 \\
469+75 \\
\end{array}$ & $\begin{array}{l}\mathrm{U} \\
02 \mathrm{~S} \\
14 \mathrm{E} \\
33 \\
\end{array}$ & $\begin{array}{ll}\mathrm{AB} & \mathrm{E} \\
\mathrm{F} & 1\end{array}$ \\
\hline $\begin{array}{r}76.04 \\
76.41 \\
125 \\
124\end{array}$ & $\begin{array}{l}\text { SAGAVANIRKTOK RIVER } \\
\text { SIDE CHANNEL } \\
\text { TAPS B/G; BLOCKPOINT }\end{array}$ & $\begin{array}{l}\mathrm{AC} ; \mathrm{BB} \\
\mathrm{BW} ; \mathrm{CA} \\
\mathrm{CN} ; \mathrm{CS} \\
\mathrm{DS} ; \mathrm{GR} ; \\
\text { HW?PS; } \\
\text { RW;S9 }\end{array}$ & SSSSSSSSCCCCSSCCCCCCSSSS & $\begin{array}{l}401498 \\
403455 \\
463+00 \\
446+00\end{array}$ & $\begin{array}{l}U: U \\
02 S: 03 S \\
14 E: 14 E \\
33: 4\end{array}$ & $\begin{array}{l}\mathrm{AB} \\
\mathrm{F}\end{array}$ \\
\hline $\begin{array}{r}78.85 \\
124\end{array}$ & $\begin{array}{l}\text { SAGAVANIRKTOK RIVER } \\
\text { SIDE CHANNEL } \\
\text { TAPS B/G; LWC }\end{array}$ & AC? GR? & jafemrapCCCCSSauseocnode & $\begin{array}{l}* 416353 \\
314+45\end{array}$ & $\begin{array}{l}U \\
03 S \\
14 \mathrm{E} \\
16 \\
\end{array}$ & $F^{B}$ I \\
\hline $\begin{array}{r}79.40 \\
124\end{array}$ & $\begin{array}{l}\text { SAGAVANIRKTOK RIVER } \\
\text { SIDE CHANNEL } \\
\text { TAPS B/G; LWC }\end{array}$ & AC? GR? & jafemrapCCCCSSauseocnode & $\begin{array}{r}* 419218 \\
285+80\end{array}$ & $\begin{array}{l}U \\
03 S \\
14 \mathrm{E} \\
17 \\
\end{array}$ & $\mathrm{~F}^{\mathrm{B}} \mathrm{I}$ \\
\hline $\begin{array}{r}79.51 \\
124\end{array}$ & $\begin{array}{l}\text { SAGAVANIRKTOK RIVER } \\
\text { SIDE CHANNEL } \\
\text { TAPS B/G; LWC }\end{array}$ & AC? GR? & jafemrapCCCCSSauseocnode & $\begin{array}{l}* 419218 \\
280+00\end{array}$ & $\begin{array}{l}\mathrm{U} \\
03 \mathrm{~S} \\
14 \mathrm{E} \\
20 \\
\end{array}$ & $\begin{array}{ll}\mathrm{B} & \\
\mathrm{F} & 1\end{array}$ \\
\hline $\begin{array}{r}79.66 \\
124\end{array}$ & $\begin{array}{l}\text { SAGAVANIRKTOK RIVER } \\
\text { SIDE CHANNEL } \\
\text { TAPS B/G; LWC }\end{array}$ & AC? GR? & jafemrapCCCCSSauseocnode & $\begin{array}{l}* 420598 \\
272+00\end{array}$ & \begin{tabular}{|l}
$U$ \\
$03 S$ \\
$14 \mathrm{E}$ \\
20
\end{tabular} & $\begin{array}{ll}\text { B } & \text { I }\end{array}$ \\
\hline $\begin{array}{r}79.94 \\
124\end{array}$ & $\begin{array}{l}\text { (Woody CK) } \\
\text { TAPS B/G; LWC }\end{array}$ & GR & jafemrapSSSSSSSSSSocnode & $\begin{array}{l}* 422115 \\
256+83\end{array}$ & $\begin{array}{l}\mathrm{U} \\
03 \mathrm{~S} \\
14 \mathrm{E} \\
20 \\
\end{array}$ & $\begin{array}{l}\mathrm{AB} \\
\mathrm{F}\end{array}$ \\
\hline $\begin{array}{r}80.34 \\
124\end{array}$ & $\begin{array}{l}\text { SAGAVANIRKTOK RIVER } \\
\text { SIDE CHANNEL } \\
\text { TAPS B/G; LWC }\end{array}$ & AC? GR & jafemrapCCCCSSauseocnode & $\begin{array}{r}* 424180 \\
236+18\end{array}$ & $\begin{array}{l}\mathrm{U} \\
03 \mathrm{~S} \\
14 \mathrm{E} \\
20\end{array}$ & F \\
\hline
\end{tabular}


USDOI BLM PMO OPEN FILE REPORT - TAPS FISH STREAMS

\begin{tabular}{|c|c|c|c|c|c|c|}
\hline MP & $\begin{array}{l}: \text { STREAM NAME(s) } \\
:\end{array}$ & $\begin{array}{l}: \text { FISH } \\
: \text { SPECIES }\end{array}$ & $\begin{array}{l}\text { A : JaFeMrApMaJuJlAuSeOcNoDe } \\
\text { D : PERIOD OF SENSITIVITY }\end{array}$ & $\begin{array}{l}\text { FIELD : } \\
\text { STATION : }\end{array}$ & $\begin{array}{l}\text { MER } \\
T\end{array}$ & $\begin{array}{l}\text { REFER } \\
\text {..ENCE }\end{array}$ \\
\hline $\mathrm{A} / \mathrm{S}$ & Comments & & $\begin{array}{l}\text { A : } \\
\text { D : }\end{array}$ & G-5 & $\begin{array}{l}: \mathrm{R} \\
: \mathrm{SEC}\end{array}$ & \\
\hline $\begin{array}{r}83.25 \\
84.22 \\
123\end{array}$ & $\begin{array}{l}\text { SAGAVANIRKTOK RIVER } \\
\text { SIDE CHANNEL } \\
\text { TAPS B/G; BLOCKPOINT }\end{array}$ & $\begin{array}{l}\mathrm{AC} ; \mathrm{BB} ; \\
\mathrm{BW} ; \mathrm{CA} ; \\
\mathrm{CN} ; \mathrm{CS} \\
\mathrm{DS} ; \mathrm{GR} ; \\
\text { HW?PS: } \\
\text { RW;S9 }\end{array}$ & SSSSSSSSCCCCSSCCCCCCSSSS & $\begin{array}{r}* 439572 \\
444700 \\
82+26 \\
58+15\end{array}$ & $\begin{array}{l}U \\
04 \mathrm{~S} \\
14 \mathrm{E} \\
5,7 \& 8\end{array}$ & $\begin{array}{l}\mathrm{AB} \\
\mathrm{F}\end{array}$ \\
\hline $\begin{array}{r}84.51 \\
123\end{array}$ & $\begin{array}{l}\text { SAGAVANIRKTOK RIVER } \\
\text { SIDE CHANNEL } \\
\text { TAPS A/G; LWC }\end{array}$ & AC? GR? & jafemrapCCCCSSauseocnode & $\begin{array}{r}446158 \\
446234 \\
42+00 \\
40+00 \\
38+00\end{array}$ & $\begin{array}{l}\mathrm{U} \\
04 \mathrm{~S} \\
14 \mathrm{E} \\
07\end{array}$ & $\begin{array}{ll} & \text { I } \\
\text { B } & \\
\text { B } & \\
\text { B } & \end{array}$ \\
\hline $\begin{array}{r}84.96 \\
123\end{array}$ & $\begin{array}{l}\text { (Dan or Charlotte CK) } \\
\text { TAPS A/G; BRIDGE \& } 2 \text { CMPs }\end{array}$ & $\begin{array}{l}\text { AC;CN; } \\
\text { GR;RW; } \\
\text { S9 }\end{array}$ & $\begin{array}{c:c}Y & \text { jafemrapCCCCSSCCCCocnode } \\
\text { E } & 448439 \text { BRE } 448509 \\
\text { S } & 448509 \text { CMP448559 } \\
& 448679 \text { CMP448739 }\end{array}$ & $\begin{array}{r}448439 \\
448739 \\
20+12\end{array}$ & $\begin{array}{l}U \\
04 S \\
14 \mathrm{E} \\
08\end{array}$ & $\begin{array}{l}\mathrm{AB} \\
\mathrm{FG}\end{array}$ \\
\hline $\begin{array}{r}86.94 \\
123\end{array}$ & $\begin{array}{l}\text { (Lori CK) } \\
\text { TAPS A/G; LWC }\end{array}$ & GR & jafemrapSSSSSSSSSSSocnode & $\begin{array}{r}459029 \\
459099 \\
1722+00\end{array}$ & $\begin{array}{l}\mathrm{U} \\
04 \mathrm{~S} \\
14 \mathrm{E} \\
29\end{array}$ & $\begin{array}{l}\mathrm{AB} \\
\mathrm{FG}\end{array}$ \\
\hline $\begin{array}{r}91.93 \\
122\end{array}$ & $\begin{array}{l}\text { (Stump CK) } \\
\text { TAPS A/G; LWC }\end{array}$ & $\begin{array}{l}\text { CN;GR; } \\
\text { LT;S9 }\end{array}$ & jafemrapSSSSSSSSSSocnode & $\begin{array}{r}485366 \\
485425 \\
1499+00\end{array}$ & $\begin{array}{l}\mathrm{U} \\
05 \mathrm{~S} \\
14 \mathrm{E} \\
16\end{array}$ & $\begin{array}{r}\mathrm{B} \\
\mathrm{FG}\end{array}$ \\
\hline $\begin{array}{c}92.19 \\
92.36 \\
122\end{array}$ & $\begin{array}{l}\text { (Clarke's Lake) } \\
\text { TAPS A/G; Causeway \& CMP }\end{array}$ & $\begin{array}{l}\text { CN;GR;LT } \\
\text { S9 }\end{array}$ & CCCCCCCCCCCCSSSSCCCCCCCC & $\begin{array}{r}486739 \\
487636 \\
1489+28 \\
1481+00 \\
\end{array}$ & $\begin{array}{l}\mathrm{U} \\
05 \mathrm{~S} \\
14 \mathrm{E} \\
16 \\
\end{array}$ & $\begin{array}{l}\mathrm{AB} \\
\mathrm{FG}\end{array}$ \\
\hline $\begin{array}{r}92.97 \\
122\end{array}$ & $\begin{array}{l}\text { SAGAVANIRKTOK RIVER } \\
\text { SIDE CHANNEL } \\
\text { TAPS A/G; LWC }\end{array}$ & $\begin{array}{l}\text { AC? CN; } \\
\text { GR;RW; } \\
\text { S9? }\end{array}$ & jafemrapCCCCSSSCCSSSnode & $\begin{array}{r}490853 \\
490922 \\
1445+25\end{array}$ & $\begin{array}{l}U \\
05 S \\
14 \mathrm{E} \\
21 \\
\end{array}$ & $\begin{array}{l}\text { A B } \\
\text { F }\end{array}$ \\
\hline $\begin{array}{r}93.36 \\
122\end{array}$ & $\begin{array}{l}\text { SAGAVANIRKTOK RIVER } \\
\text { SIDE CHANNEL } \\
\text { TAPS B/G; LWC }\end{array}$ & $\begin{array}{l}\text { AC? CN?; } \\
\text { GR;S9 }\end{array}$ & jafemrapCCCCSSSCCSSSnode & $\begin{array}{r}492917 \\
492973 \\
1424+79\end{array}$ & $\begin{array}{l}\mathrm{U} \\
05 \mathrm{~S} \\
14 \mathrm{E} \\
21 \\
\end{array}$ & $\begin{array}{l}\mathrm{AB} \\
\mathrm{F}\end{array}$ \\
\hline $\begin{array}{r}95.79 \\
121\end{array}$ & $\begin{array}{l}\text { (Arthur CK) } \\
\text { TAPS A/G; LWC }\end{array}$ & $\begin{array}{l}\text { AC;BB; } \\
\text { CN;GR }\end{array}$ & jafemrapCCCCSSSCCCocnode & $\begin{array}{r}505760 \\
505825 \\
1297+50\end{array}$ & $\begin{array}{l}U \\
05 S \\
14 \mathrm{E} \\
32 \\
\end{array}$ & $\begin{array}{l}\mathrm{AB} \\
\mathrm{FG}\end{array}$ \\
\hline $\begin{array}{r}96.12 \\
121\end{array}$ & $\begin{array}{l}\text { (Gustafson Gulch) } \\
\text { TAPS A/G; LWC }\end{array}$ & $\begin{array}{l}\text { AC;BB; } \\
\text { CN;GR }\end{array}$ & jafemrapCCCCSSSCCCocnode & $\begin{array}{r}507485 \\
507550 \\
1280+00\end{array}$ & $\begin{array}{l}U \\
06 S \\
14 \mathrm{E} \\
05\end{array}$ & $\begin{array}{l}\mathrm{AB} \\
\mathrm{FG}\end{array}$ \\
\hline $\begin{array}{r}96.6 \\
121\end{array}$ & $\begin{array}{l}\text { (Gustafson Gulch) } \\
\text { TAPS A/G; TAPS DOES NOT } \\
\text { CROSS }\end{array}$ & $\overline{\mathrm{GR}}$ & jafemrapCCCCSSSCCCocnode & $\begin{array}{l}\text { NO TAPS } \\
\text { X1NG } \\
1255+00\end{array}$ & $\begin{array}{l}U \\
06 S \\
14 E \\
06\end{array}$ & $\mathbf{F}$ \\
\hline $\begin{array}{r}99.07 \\
121\end{array}$ & $\begin{array}{l}\text { (Polygon CK) } \\
\text { TAPS AVG; LWC }\end{array}$ & $\begin{array}{l}\mathrm{AC} ; \mathrm{BB} \\
\mathrm{CN} ; \mathrm{GR}\end{array}$ & jafemrapCCCCSSSCCCocnode & $\begin{array}{r}523070 \\
523145 \\
1125+03\end{array}$ & $\begin{array}{l}U \\
06 S \\
14 \mathrm{E} \\
19\end{array}$ & $\begin{array}{l}\mathrm{AB} \\
\mathrm{FG}\end{array}$ \\
\hline $\begin{array}{r}99.99 \\
120\end{array}$ & $\begin{array}{l}\text { (Poison Pipe CK) } \\
\text { TAPS AVG; LWC }\end{array}$ & $\begin{array}{l}\mathrm{AC} ; \mathrm{CN} \\
\mathrm{GR}\end{array}$ & jafemrapCCCCSSSCCSSSocnode & $\begin{array}{r}527926 \\
527986 \\
1077+10\end{array}$ & $\begin{array}{l}U \\
06 S \\
14 \mathrm{E} \\
19\end{array}$ & $\begin{array}{l}\mathrm{AB} \\
\mathrm{FG}\end{array}$ \\
\hline 100.31 & $\begin{array}{l}\text { (Climb CK) } \\
\text { TAPS A/G; CMP }\end{array}$ & AC;GR & jafemrapCCCCSSSCCCocnode & $\begin{array}{r}529599 \\
529669 \\
1060+34\end{array}$ & $\begin{array}{l}U \\
06 \mathrm{~S} \\
14 \mathrm{E} \\
30\end{array}$ & $\begin{array}{l}\mathrm{AB} \\
\mathrm{FG}\end{array}$ \\
\hline
\end{tabular}




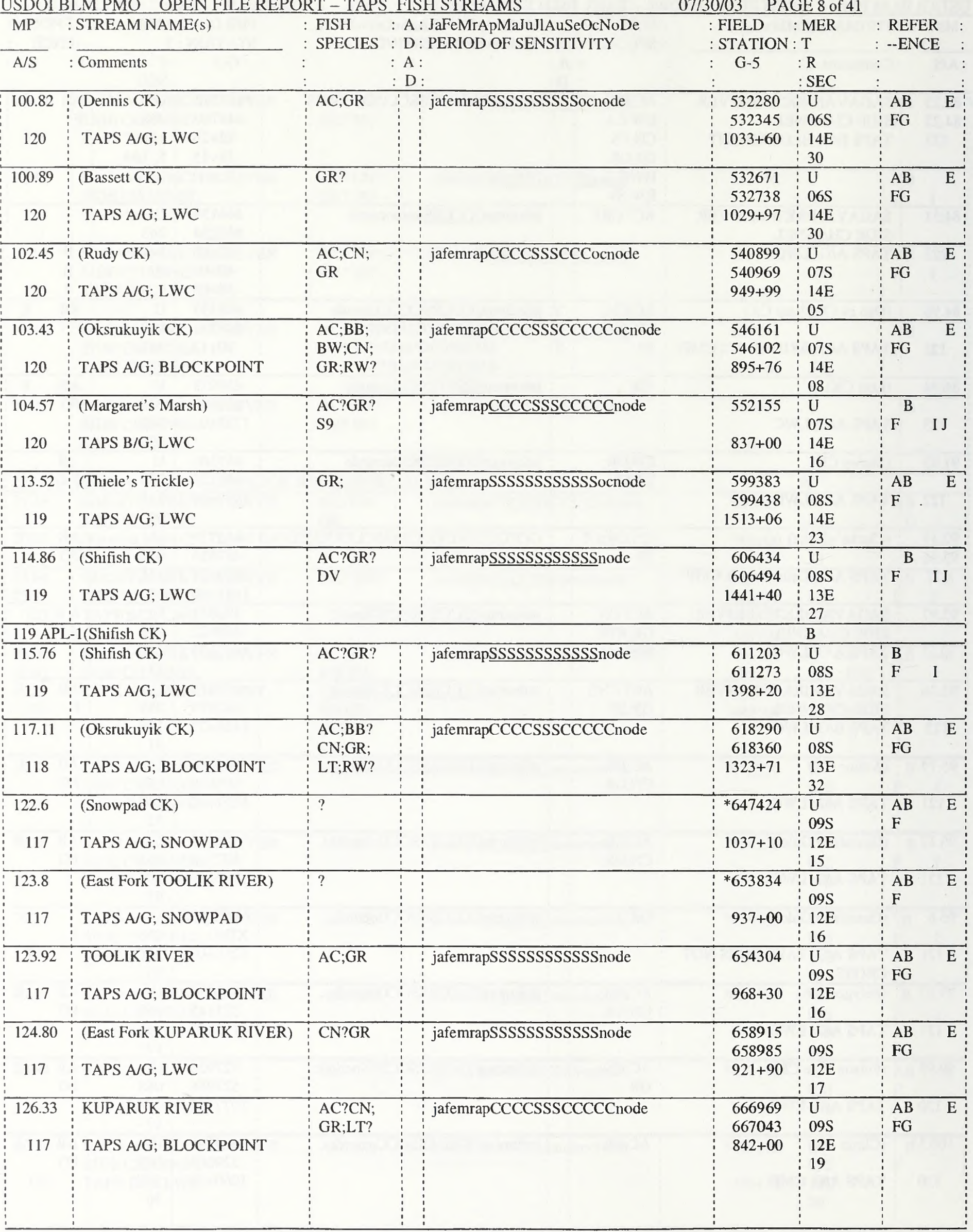


USDOI BLM PMO OPEN FILE REPORT - TAPS FISH STREAMS 07/30/03 PAGE 9 of 41

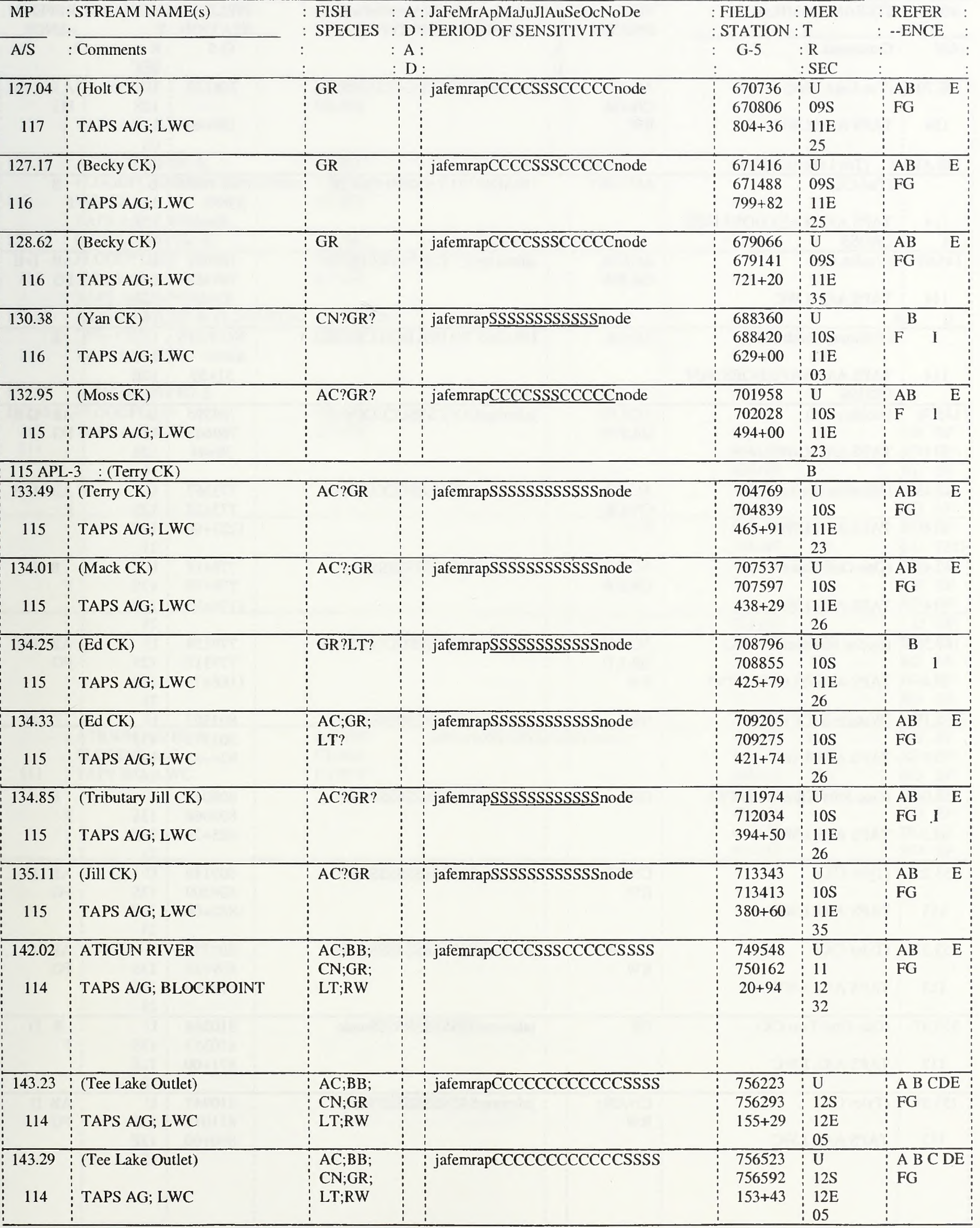


USDOI BLM PMO OPEN FILE REPORT - TAPS FISH STREAMS

\begin{tabular}{|c|c|c|c|c|c|c|}
\hline MP & STREAM NAME(s) & $\begin{array}{l}\text { F1SH } \\
\text { SPECIES }\end{array}$ & $\begin{array}{l}\text { A : JaFeMrApMaJuJlAuSeOcNoDe } \\
\text { D : PERIOD OF SENSITIVITY }\end{array}$ & $\begin{array}{l}\text { : FIELD } \\
: \text { STATION }\end{array}$ & $\begin{array}{l}\text { MER } \\
\mathrm{T}\end{array}$ & $\begin{array}{l}\text { REFER } \\
\text {--ENCE }\end{array}$ \\
\hline $\mathrm{A} / \mathrm{S}$ & : Comments & & $\begin{array}{l}\text { A : } \\
\text { D : }\end{array}$ & $: \quad$ G-5 & $\begin{array}{l}: \mathrm{R} \\
: \mathrm{SEC}\end{array}$ & \\
\hline $\begin{array}{r}143.70 \\
114\end{array}$ & $\begin{array}{l}\text { (Tee Lake lnlet) } \\
\text { TAPS A/G; LWC }\end{array}$ & $\begin{array}{l}\mathrm{AC} ; \mathrm{BB} \\
\mathrm{CN} ; \mathrm{GR} \\
\mathrm{RW}\end{array}$ & jafemrapCCCCCCCCCCCCSSSS & $\begin{array}{l}758730 \\
130+60\end{array}$ & $\begin{array}{l}\mathrm{U} \\
12 \mathrm{~S} \\
12 \mathrm{E} \\
05\end{array}$ & $\begin{array}{l}\text { A B C DE } \\
\text { FG }\end{array}$ \\
\hline $114 \mathrm{APS}$ & $-2 \quad$ (Tee Lake Inlet) & & & & $\overline{B C D}$ & \\
\hline 114 & $\begin{array}{l}\text { (Tad CK) } \\
\text { TAPS A/G; TAPS DOES NOT } \\
\text { CROSS }\end{array}$ & AC? GR? & DRAINS TO VANISH CREEK & $\begin{array}{l}\text { NO TAPS } \\
\text { X1NG } \\
40+43\end{array}$ & $\begin{array}{l}\mathrm{U} \\
12 \mathrm{~S} \\
12 \mathrm{E} \\
16\end{array}$ & $\mathrm{~F}^{\mathrm{B}}$ \\
\hline $\begin{array}{r}145.67 \\
114\end{array}$ & $\begin{array}{l}\text { (Vanish CK) } \\
\text { TAPS A/G; LWC }\end{array}$ & $\begin{array}{l}\mathrm{AC} ; \mathrm{CN} ; \\
\mathrm{GR} ; \mathrm{RW}\end{array}$ & jafemrapCCCCSSSCCCCCnode & $\begin{array}{c}769092 \\
769162 \\
35+80\end{array}$ & $\begin{array}{l}U \\
12 S \\
12 E \\
16 \\
\end{array}$ & $\begin{array}{l}\mathrm{AB} \quad \mathrm{DE} \\
\mathrm{FG}\end{array}$ \\
\hline $1 \mathrm{I} 4$ & $\begin{array}{l}\text { (Tributary Holden CK) } \\
\text { TAPS A/G; TAPS DOES NOT } \\
\text { CROSS }\end{array}$ & AC;GR & DRAINS TO HOLDEN CREEK & $\begin{array}{l}\text { NO TAPS } \\
\text { XING } \\
31+59\end{array}$ & $\begin{array}{l}\mathrm{U} \\
12 \mathrm{~S} \\
12 \mathrm{E} \\
16 \\
\end{array}$ & B \\
\hline $\begin{array}{c}145.76 \\
114\end{array}$ & $\begin{array}{l}\text { (Holden CK) } \\
\text { TAPS A/G; LWC }\end{array}$ & $\begin{array}{l}\mathrm{AC} ; \mathrm{CN} ; \\
\mathrm{GR} ; \mathrm{RW}\end{array}$ & jafemrapCCCCSSSCCCCCnode & $\begin{array}{l}769595 \\
769665 \\
30+44\end{array}$ & $\begin{array}{l}\mathrm{U} \\
12 \mathrm{~S} \\
12 \mathrm{E} \\
16 \\
\end{array}$ & $\begin{array}{l}\mathrm{AB} \quad \mathrm{DE} \\
\mathrm{FG}\end{array}$ \\
\hline $\begin{array}{c}146.48 \\
114\end{array}$ & $\begin{array}{l}\text { (Mainline Spring CK) } \\
\text { TAPS A/G; LWC }\end{array}$ & $\begin{array}{l}\text { AC;BB; } \\
\text { CN;GR; } \\
\text { RW }\end{array}$ & jafemrapCCCCSSSCCCCCnode & $\begin{array}{r}773367 \\
773437 \\
1227+00\end{array}$ & $\begin{array}{l}\mathrm{U} \\
12 \mathrm{~S} \\
12 \mathrm{E} \\
21 \\
\end{array}$ & $\begin{array}{l}\mathrm{AB} \quad \mathrm{DE} \\
\mathrm{F}\end{array}$ \\
\hline $\begin{array}{c}147.43 \\
114\end{array}$ & $\begin{array}{l}\text { (One-One-Three CK) } \\
\text { TAPS A/G; LWC }\end{array}$ & $\begin{array}{l}\mathrm{AC} ; \mathrm{CN} ; \\
\mathrm{GR} ; \mathrm{RW}\end{array}$ & jafemrapSSSSSSSSSSSSnode & $\begin{array}{r}778418 \\
778478 \\
1176+95\end{array}$ & $\begin{array}{l}U \\
12 S \\
12 E \\
28 \\
\end{array}$ & B D \\
\hline $\begin{array}{c}147.58 \\
114\end{array}$ & $\begin{array}{l}\text { (Roche Mountonnee CK) } \\
\text { TAPS A/G; BLOCKPOINT }\end{array}$ & $\begin{array}{l}\text { AC;CN; } \\
\text { GR;LT; } \\
\text { RW }\end{array}$ & jafemrapCCCCSSSCCCCCnode & $\begin{array}{r}779139 \\
779318 \\
1168+75\end{array}$ & $\begin{array}{l}\mathrm{U} \\
12 \mathrm{~S} \\
12 \mathrm{E} \\
28 \\
\end{array}$ & $\begin{array}{l}\text { AB } \quad \text { DE } \\
\text { FG }\end{array}$ \\
\hline $\begin{array}{c}152.19 \\
113\end{array}$ & $\begin{array}{l}\text { (Waterhole CK) } \\
\text { TAPS AVG; LWC }\end{array}$ & GR & jafemrapSSSSSSSSSSSSnode & $\begin{array}{l}803507 \\
803572 \\
924+83\end{array}$ & $\begin{array}{l}\mathrm{U} \\
13 \mathrm{~S} \\
12 \mathrm{E} \\
16 \\
\end{array}$ & F D \\
\hline $\begin{array}{c}153.04 \\
113\end{array}$ & $\begin{array}{l}\text { (One-Fifty-Three Mile CK) } \\
\text { TAPS A/G; LWC }\end{array}$ & GR & jafemrapSSSSSSSSSSSSnode & $\begin{array}{l}808015 \\
808068 \\
885+24\end{array}$ & $\begin{array}{l}\mathrm{U} \\
13 \mathrm{~S} \\
12 \mathrm{E} \\
21 \\
\end{array}$ & B \\
\hline $\begin{array}{c}153.25 \\
113\end{array}$ & $\begin{array}{l}\text { (Tyler CK) } \\
\text { TAPS A/G; LWC }\end{array}$ & $\begin{array}{l}\mathrm{CN} ; \mathrm{GR} \\
\mathrm{RW}\end{array}$ & jafemrapSSSSSSSSSSSSnode & $\begin{array}{l}809149 \\
809209 \\
882+00\end{array}$ & $\begin{array}{l}\mathrm{U} \\
13 \mathrm{~S} \\
12 \mathrm{E} \\
21 \\
\end{array}$ & $\begin{array}{l}\mathrm{AB} \quad \mathrm{D} \\
\text { FG }\end{array}$ \\
\hline $\begin{array}{c}153.33 \\
113\end{array}$ & $\begin{array}{l}\text { (Tyler CK) } \\
\text { TAPS AGG; LWC }\end{array}$ & $\begin{array}{l}\mathrm{CN} ; \mathrm{GR} \\
\mathrm{RW}\end{array}$ & jafemrapSSSSSSSSSSSSnode & $\begin{array}{l}809558 \\
809628 \\
879+00\end{array}$ & $\begin{array}{l}\mathrm{U} \\
13 \mathrm{~S} \\
12 \mathrm{E} \\
28 \\
\end{array}$ & $\begin{array}{l}\mathrm{AB} \quad \mathrm{D} \\
\mathrm{FG}\end{array}$ \\
\hline $\begin{array}{r}153.47 \\
112\end{array}$ & $\begin{array}{l}\text { (One-One-Two CK) } \\
\text { TAPS A/G; LWC }\end{array}$ & GR & jafemrapSSSSSSSSSSSSnode & $\begin{array}{l}810288 \\
810353 \\
871+00\end{array}$ & $\begin{array}{l}\mathrm{U} \\
13 \mathrm{~S} \\
12 \mathrm{E} \\
28 \\
\end{array}$ & \begin{tabular}{|l} 
B D \\
F
\end{tabular} \\
\hline $\begin{array}{c}153.59 \\
112\end{array}$ & $\begin{array}{l}\text { (Tyler CK) } \\
\text { TAPS A/G; LWC }\end{array}$ & $\begin{array}{l}\text { CN;GR; } \\
\text { RW }\end{array}$ & jafemrapSSSSSSSSSSSSnode & $\begin{array}{l}810947 \\
811012 \\
860+00\end{array}$ & $\begin{array}{l}U \\
13 S \\
12 E \\
28\end{array}$ & $\begin{array}{l}\text { AB D } \\
\text { FG }\end{array}$ \\
\hline
\end{tabular}


USDOI BLM PMO OPEN FILE REPORT - TAPS FISH STREAMS

07/30/03 PAGE 11 of 41

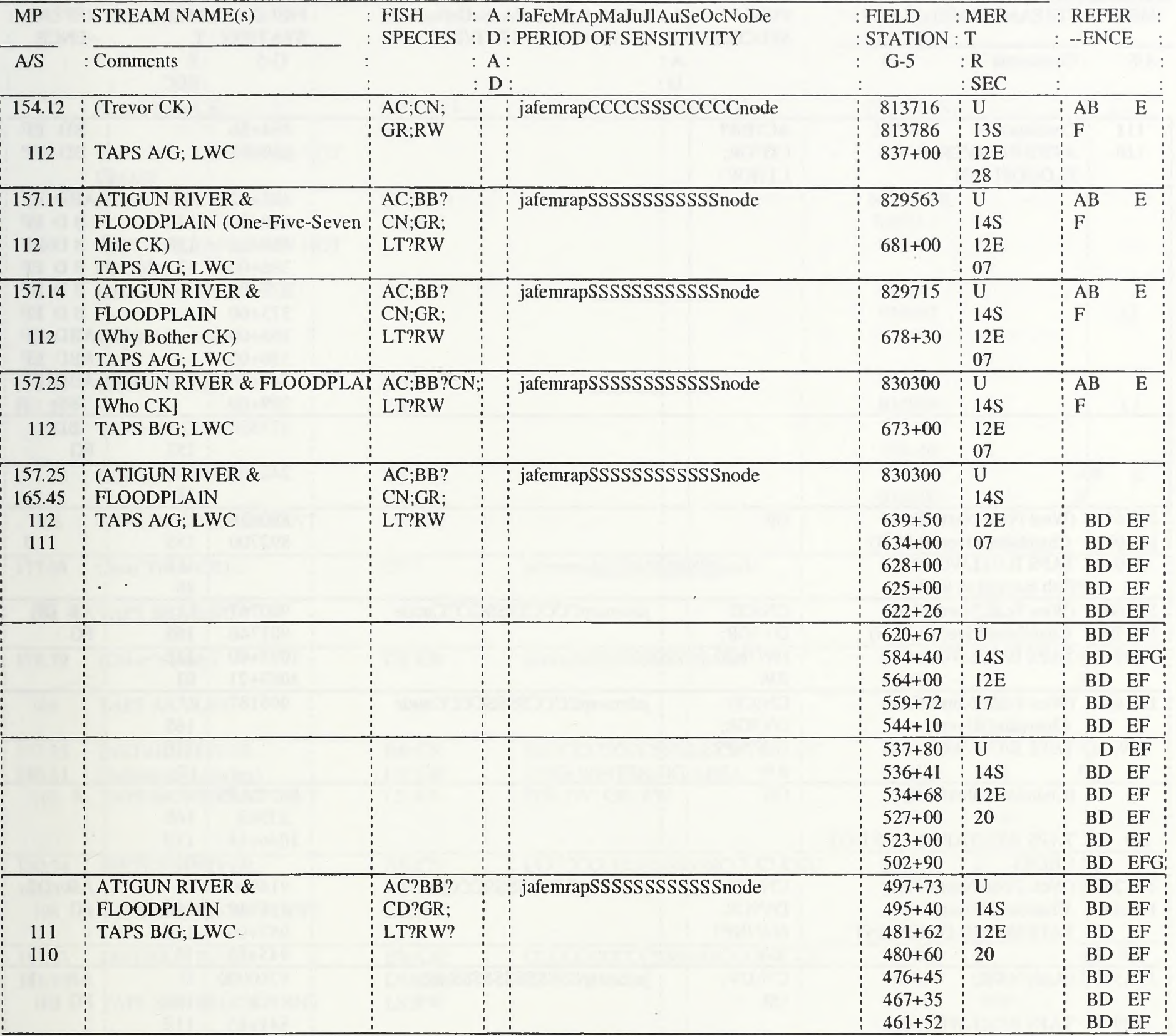




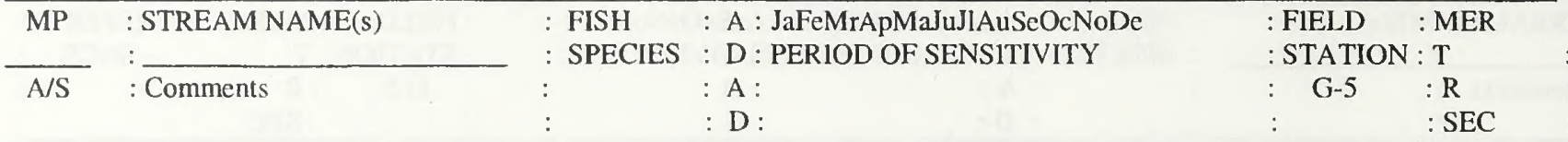

\begin{tabular}{|c|c|c|c|c|c|c|}
\hline $\begin{array}{l}111 \\
110\end{array}$ & $\begin{array}{l}\text { Continued } \\
\text { ATIGUN RIVER \& } \\
\text { FLOODPLAIN }\end{array}$ & $\begin{array}{l}\text { AC?BB? } \\
\text { CD?GR; } \\
\text { LT?RW? }\end{array}$ & & $\begin{array}{l}454+56 \\
450+67\end{array}$ & & $\begin{array}{ll}\text { BD } & \text { EF } \\
\text { BD } & \text { EF }\end{array}$ \\
\hline & & & & $\begin{array}{l}428+42 \\
410+00 \\
409+25 \\
386+00 \\
377+00 \\
373+60 \\
368+00 \\
350+00 \\
347+50 \\
299+00 \\
\end{array}$ & $\begin{array}{l}\mathrm{U} \\
14 \mathrm{~S} \\
12 \mathrm{E} \\
32\end{array}$ & $\begin{array}{l}\text { ABD EF } \\
\text { B D EF } \\
\text { B D EF } \\
\text { B D EF } \\
\text { B D EF } \\
\text { B D EF } \\
\text { ABD EF } \\
\text { ABD EF } \\
\text { ABD EF } \\
\text { BD F }\end{array}$ \\
\hline & & & & $\begin{array}{l}873550 \\
242+00\end{array}$ & $\begin{array}{l}\mathrm{U} \\
15 \mathrm{~S} \\
12 \mathrm{E} \\
18\end{array}$ & $\begin{array}{l}\text { BD } \\
F G\end{array}$ \\
\hline $\begin{array}{c}168.64 \\
169.07 \\
110\end{array}$ & $\begin{array}{l}\text { (West Fork, North Fork, } \\
\text { Chandalar River FLPLN) } \\
\text { TAPS B/G; LWC } \\
\text { Fish parallel to ROW }\end{array}$ & GR & . & $\begin{array}{l}890436 \\
892700\end{array}$ & $\begin{array}{l}U \\
15 S \\
11 E \\
26\end{array}$ & J \\
\hline $\begin{array}{c}170.60 \\
170.79 \\
109\end{array}$ & $\begin{array}{l}\text { (West Fork, North Fork, } \\
\text { Chandalar River FLPLN) } \\
\text { TAPS B/G; LWC }\end{array}$ & $\begin{array}{l}\text { CN;CI? } \\
\text { DV?GR; } \\
\text { HW?NP? } \\
\text { RW }\end{array}$ & $\begin{array}{l}\text { jafemrapCCCCSSSSCCCCnode } \\
\vdots\end{array}$ & $\begin{array}{r}900767 \\
901746 \\
1093+00 \\
1083+21 \\
\end{array}$ & $\begin{array}{l} \\
16 S \\
11 E \\
03\end{array}$ & $\begin{array}{l}\text { AB DE } \\
\text { FG }\end{array}$ \\
\hline $\begin{array}{c}171.44 \\
109\end{array}$ & $\begin{array}{l}\text { (West Fork, North Fork, } \\
\text { Chandalar River) } \\
\text { TAPS B/G; LWC }\end{array}$ & $\begin{array}{l}\text { CN;CI? } \\
\text { DV?GR; } \\
\text { HW?NP? } \\
\text { RW }\end{array}$ & jafemrapCCCCSSSSCCCCnode & 905187 & $\begin{array}{l}\mathrm{U} \\
16 \mathrm{~S} \\
11 \mathrm{E} \\
03\end{array}$ & \\
\hline 109 & $\begin{array}{l}\text { (Chandalar Shelf CK) } \\
\text { TAPS B/G; TAPS DOES NOT } \\
\text { CROSS }\end{array}$ & GR & & $\begin{array}{l}\text { NO TAPS } \\
\text { XING } \\
1046+14\end{array}$ & $\begin{array}{l}\mathrm{U} \\
16 \mathrm{~S} \\
11 \mathrm{E} \\
03 \\
\end{array}$ & $\bar{B}$ \\
\hline $\begin{array}{c}173.28 \\
173.44 \\
109 \\
\end{array}$ & $\begin{array}{l}\text { (West Fork, North Fork, } \\
\text { Chandalar River) } \\
\text { TAPS B/G; BLOCKPOINT }\end{array}$ & $\begin{array}{l}\text { CN;CI? } \\
\text { DV?GR; } \\
\text { HW?NP? } \\
\text { RW }\end{array}$ & jafemrapCCCCSSSSCCCCnode & $\begin{array}{l}914900 \\
915740 \\
957+00 \\
945+23 \\
\end{array}$ & $\begin{array}{l}\mathrm{U} \\
16 \mathrm{~S} \\
11 \mathrm{E} \\
16 \\
\end{array}$ & $\begin{array}{l}\text { AB DE } \\
\text { FG }\end{array}$ \\
\hline $\begin{array}{c}175.38 \\
109\end{array}$ & $\begin{array}{l}\text { (Andy's CK) } \\
\text { TAPS B/G; LWC }\end{array}$ & $\begin{array}{l}\text { CN;DV; } \\
\text { GR }\end{array}$ & jafemrapSSSSSSSSSSSSnode & $\begin{array}{l}9260000 \\
841+65 \\
840+52 \\
\end{array}$ & $\begin{array}{l}U \\
16 S \\
11 \mathrm{E} \\
20 \\
\end{array}$ & $\begin{array}{l}\mathrm{AB} \quad \mathrm{E} \\
\mathrm{FG}\end{array}$ \\
\hline $\begin{array}{c}175.56 \\
109\end{array}$ & $\begin{array}{l}\text { (Truck Stop CK) } \\
\text { TAPS B/G; LWC }\end{array}$ & $\begin{array}{l}\text { CD;DV; } \\
\text { GR?RW? }\end{array}$ & jafemrapSSSSSSSSSSSSnode & $\begin{array}{l}926950 \\
832+56\end{array}$ & $\begin{array}{l}\mathrm{U} \\
16 \mathrm{~S} \\
11 \mathrm{E} \\
20 \\
\end{array}$ & B 1 \\
\hline $\begin{array}{c}175.76 \\
109\end{array}$ & $\begin{array}{l}\text { (Truck Stop CK) } \\
\text { TAPS B/G; LWC }\end{array}$ & $\begin{array}{l}\text { CD?DV? } \\
\text { GR?RW? }\end{array}$ & jafemrapSSSSSSSSSSSSnode & $\begin{array}{l}928000 \\
824+20\end{array}$ & $\begin{array}{l}\mathrm{U} \\
16 \mathrm{~S} \\
11 \mathrm{E} \\
30 \\
\end{array}$ & $\begin{array}{ll}\text { B } & \\
1 & 1\end{array}$ \\
\hline $\begin{array}{c}175.81 \\
109\end{array}$ & $\begin{array}{l}\text { (Truck Stop CK) } \\
\text { TAPS B/G: LWC }\end{array}$ & $\begin{array}{l}\text { CD;DV; } \\
\text { GR?RW? }\end{array}$ & jafemrapSSSSSSSSSSSSnode & $\begin{array}{l}928300 \\
822+41\end{array}$ & $\begin{array}{l}U \\
16 S \\
11 \mathrm{E} \\
30 \\
\end{array}$ & B I \\
\hline 109 & $\begin{array}{l}\text { (Truck Stop CK) } \\
\text { TAPS B/G: TAPS DOES NOT } \\
\text { CROSS }\end{array}$ & $\begin{array}{l}\text { CD?;DV; } \\
\text { GR;RW }\end{array}$ & jafemrapSSSSSSSSSSSSnode & $\begin{array}{l}\text { NO TAPS } \\
\text { XING } \\
820+73\end{array}$ & $\begin{array}{l}U \\
16 S \\
11 E \\
30\end{array}$ & $\mathrm{~B}$ \\
\hline
\end{tabular}


USDOI BLM PMO OPEN FILE REPORT - TAPS FISH STREAMS

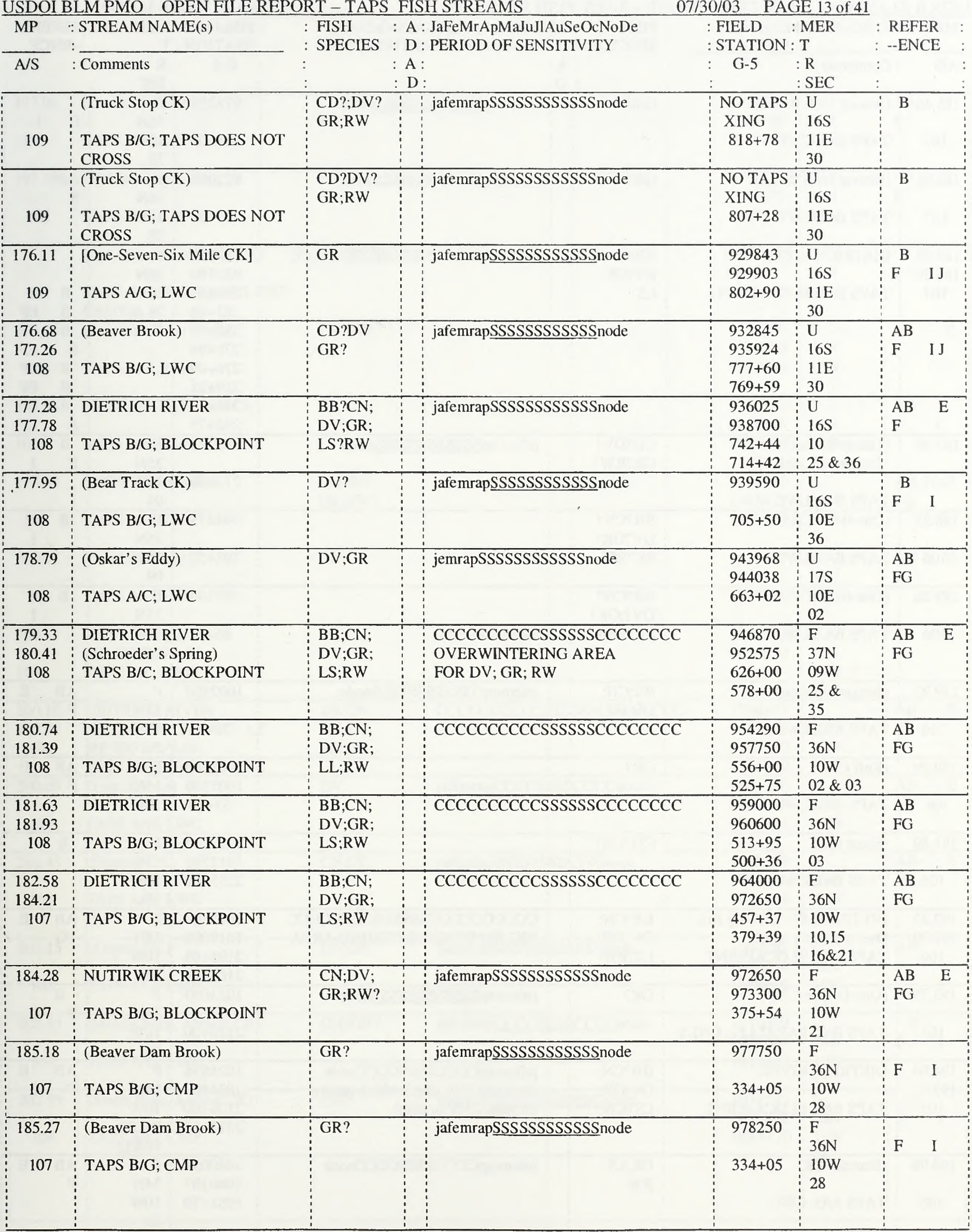




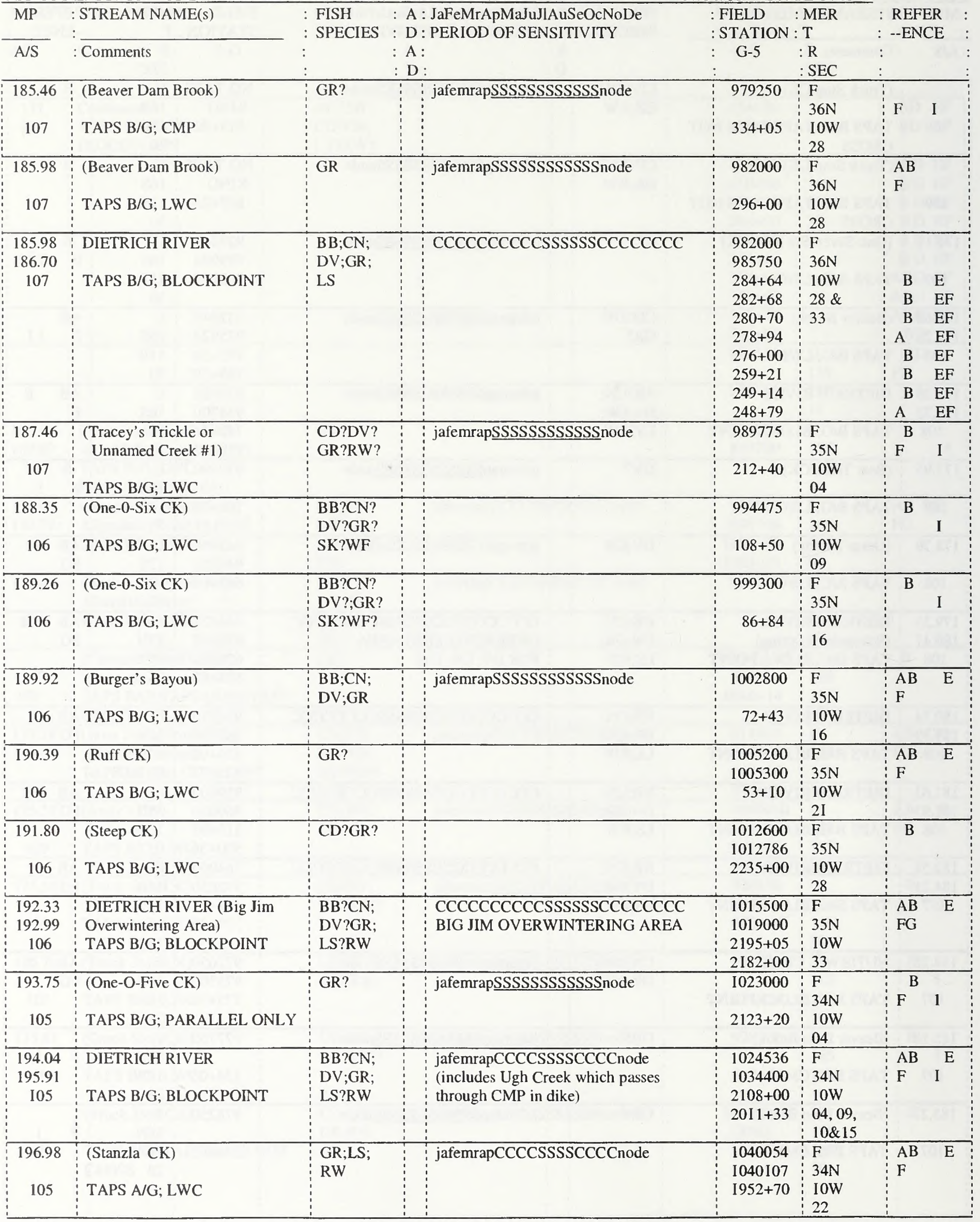


USDOI BLM PMO OPEN FILE REPORT - TAPS FISH STREAMS

07/30/03 PAGE 15 of 41

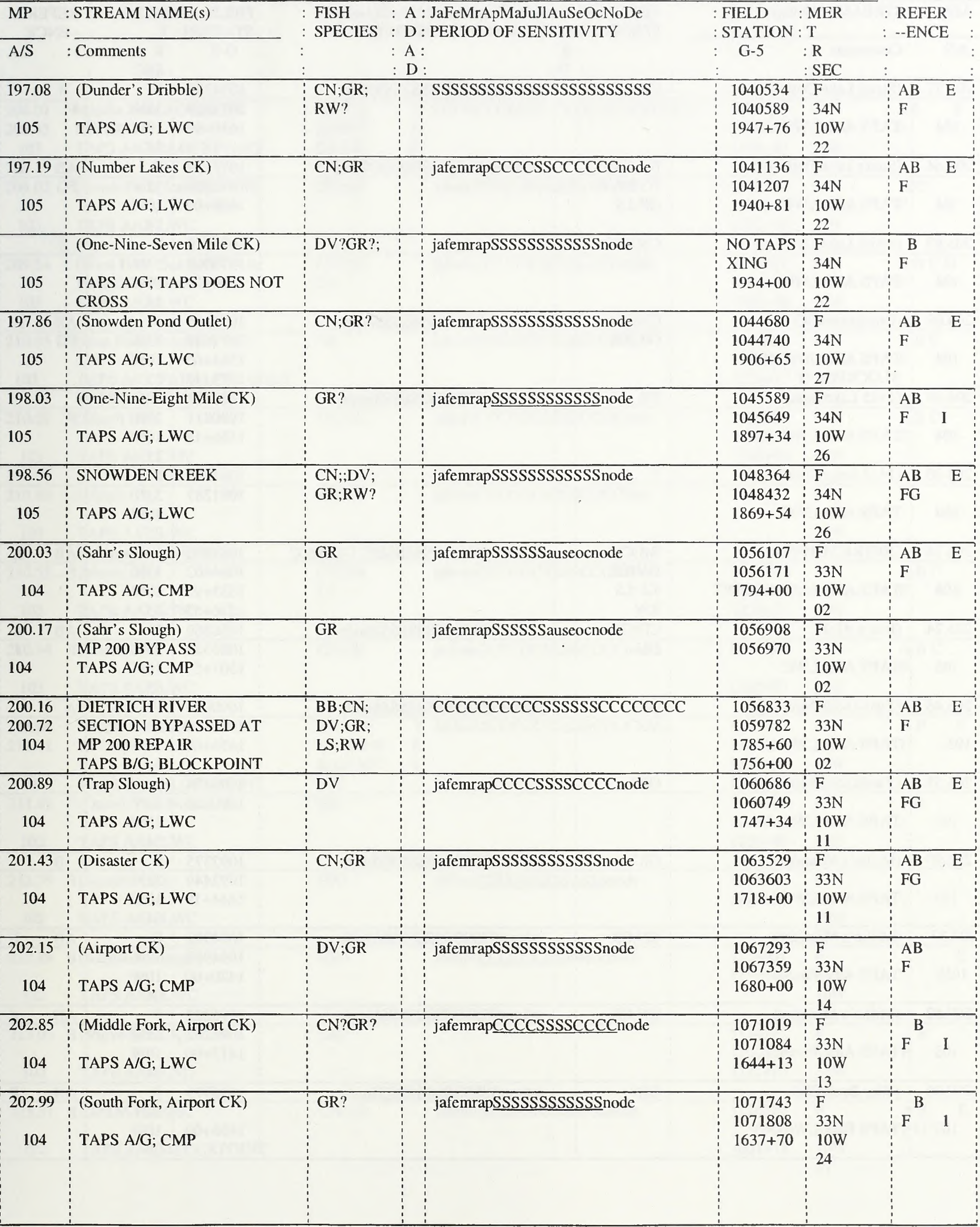


USDOI BLM PMO OPEN FILE REPOR'T - TAPS FISH STREAMS

07/30/03 PAGE 16 of 41

\begin{tabular}{|c|c|c|c|c|c|c|}
\hline MP & STREAM NAME(s) & $\begin{array}{l}\text { FISH } \\
\text { SPECIES }\end{array}$ & $\begin{array}{l}\text { A : JaFeMrApMaJuJlAuSeOcNoDe } \\
\text { D : PERIOD OF SENSITIVITY }\end{array}$ & $\begin{array}{l}\text { :FIELD } \\
\text { STATION }\end{array}$ & $\begin{array}{l}\text { MER } \\
T\end{array}$ & $\begin{array}{l}\text { REFER } \\
\text {--ENCE }\end{array}$ \\
\hline $\mathrm{A} / \mathrm{S}$ & Comments & & & G-5 & $\begin{array}{l}: \mathrm{R} \\
: \text { SEC }\end{array}$ & \\
\hline $\begin{array}{c}203.57 \\
104\end{array}$ & $\begin{array}{l}\text { (Steitz Lake Outlet) } \\
\text { TAPS A/G; LWC }\end{array}$ & GR & jafemrapSSSSSSSSSSSSnode & $\begin{array}{l}1074799 \\
1074869 \\
1610+00\end{array}$ & $\begin{array}{l}F \\
33 \mathrm{~N} \\
10 \mathrm{~W} \\
24\end{array}$ & F \\
\hline $\begin{array}{c}203.64 \\
104\end{array}$ & $\begin{array}{l}\text { (Steitz Lake Outlet) } \\
\text { TAPS A/G; LWC }\end{array}$ & $\begin{array}{l}\text { BB;CN; } \\
\text { DS?DV? } \\
\text { GR;LS }\end{array}$ & jafemrapCCCCSSSSCCCCnode & $\begin{array}{l}1075159 \\
1075229 \\
1608+00\end{array}$ & $\begin{array}{l}F \\
33 N \\
10 W \\
24\end{array}$ & $\begin{array}{ll}\mathrm{AB} & \mathrm{E} \\
\mathrm{FG} & \end{array}$ \\
\hline $\begin{array}{c}203.80 \\
104\end{array}$ & $\begin{array}{l}\text { (Steitz Lake Overflow) } \\
\text { TAPS A/G; LWC }\end{array}$ & CN;GR & & $\begin{array}{l}1076010 \\
1076069 \\
1600+00\end{array}$ & $\begin{array}{l}F \\
33 N \\
10 W \\
24\end{array}$ & B \\
\hline $\begin{array}{c}204.08 \\
104\end{array}$ & $\begin{array}{l}\text { (Brockman CK) } \\
\text { TAPS A/G; SUMMER } \\
\text { BLOCKPOINT }\end{array}$ & $\begin{array}{l}\mathrm{CN} ; \mathrm{DV} \\
\mathrm{GR} ; \mathrm{RW}\end{array}$ & jafemrapSSSSSSSSSSSSnode & $\begin{array}{l}1077385 \\
1077679 \\
1581+65 \\
1579+18\end{array}$ & $\begin{array}{l}\mathrm{F} \\
33 \mathrm{~N} \\
10 \mathrm{~W} \\
25\end{array}$ & $\begin{array}{l}\mathrm{AB} \\
\mathrm{FG}\end{array}$ \\
\hline $\begin{array}{c}204.69 \\
104\end{array}$ & $\begin{array}{l}\text { (1415 Lake Inlet) } \\
\text { TAPS A/G; LWC }\end{array}$ & $\overline{\mathrm{GR}}$ & jafemrapSSSSSSSSSSSSnode & $\begin{array}{l}1080751 \\
1080811 \\
1556+18\end{array}$ & $\begin{array}{l}F \\
33 N \\
10 W \\
25\end{array}$ & F \\
\hline $\begin{array}{l}204.80 \\
104\end{array}$ & $\begin{array}{l}\text { (1415 Lake Outlet) } \\
\text { TAPS A/G; LWC }\end{array}$ & $?$ & & $\begin{array}{l}1081362 \\
1081287\end{array}$ & $\begin{array}{l}\mathrm{F} \\
33 \mathrm{~N} \\
10 \mathrm{~W} \\
25\end{array}$ & \\
\hline $\begin{array}{c}205.34 \\
104\end{array}$ & $\begin{array}{l}\text { DIETRICH RIVER } \\
\text { TAPS A/G; BLOCKPOINT }\end{array}$ & $\begin{array}{l}\mathrm{BB} ; \mathrm{CN} \\
\mathrm{DV} ? \mathrm{GR} \\
\mathrm{KS} ? \mathrm{LS} \\
\mathrm{RW}\end{array}$ & CCCCCCCCCSSSSSSCCCCCCCC & $\begin{array}{l}1084002 \\
1084402 \\
1533+95 \\
1526+55 \\
\end{array}$ & $\begin{array}{l}\mathrm{F} \\
33 \mathrm{~N} \\
10 \mathrm{~W} \\
35 \\
\end{array}$ & $\begin{array}{l}\mathrm{AB} \\
\mathrm{F}\end{array}$ \\
\hline $\begin{array}{l}205.74 \\
103\end{array}$ & $\begin{array}{l}\text { (Eva's Alv) } \\
\text { TAPS A/G; LWC }\end{array}$ & $\begin{array}{l}\text { CD;GR } \\
\text { DV }\end{array}$ & jafemrSSSSSSSSSSSSSSnode & $\begin{array}{l}1086260 \\
1086330 \\
1507+55\end{array}$ & $\begin{array}{l}F \\
33 N \\
10 W \\
35 \\
\end{array}$ & $\mathrm{AB}$ \\
\hline $\begin{array}{l}206.46 \\
103\end{array}$ & $\begin{array}{l}\text { (Two-O-Six Mile CK) } \\
\text { TAPS A/G; LWC }\end{array}$ & GR? & jafemrSSSSSSSSSSSSSSnode & $\begin{array}{l}1090099 \\
1090164 \\
1450+60\end{array}$ & $\begin{array}{l}F \\
33 \mathrm{~N} \\
10 \mathrm{~W} \\
34\end{array}$ & $\overline{\mathrm{A}}$ \\
\hline $\begin{array}{c}206.53 \\
103\end{array}$ & $\begin{array}{l}\text { (Two-O-Six Mile CK) } \\
\text { TAPS A/G; LWC }\end{array}$ & GR? & & $\begin{array}{l}1090454 \\
1090529\end{array}$ & $\begin{array}{l}\mathrm{F} \\
33 \mathrm{~N} \\
10 \mathrm{~W} \\
34 \\
\end{array}$ & \\
\hline $\begin{array}{c}206.90 \\
103\end{array}$ & $\begin{array}{l}\text { (Millie's Meander) } \\
\text { TAPS A/G; LWC }\end{array}$ & GR? & jafemrSSSSSSSSSSSSSSnode & $\begin{array}{l}1092375 \\
1092440 \\
1444+19\end{array}$ & $\begin{array}{l}\mathrm{F} \\
32 \mathrm{~N} \\
10 \mathrm{~W} \\
04\end{array}$ & $\mathrm{~F}^{\mathrm{B}}$ \\
\hline $\begin{array}{c}207.37 \\
103\end{array}$ & $\begin{array}{l}\text { (Millie's Meander) } \\
\text { TAPS A/G; Parallel }\end{array}$ & $\mathrm{CN} ; \mathrm{GR}$ & jafemrSSSSSSSSSSSSSSnode & $\begin{array}{l}1094902 \\
1094962 \\
1420+00\end{array}$ & $\begin{array}{l}\mathrm{F} \\
32 \mathrm{~N} \\
10 \mathrm{~W} \\
04 \\
\end{array}$ & $\begin{array}{l}\mathrm{AB} \\
\mathrm{F}\end{array}$ \\
\hline $\begin{array}{c}207.62 \\
103\end{array}$ & $\begin{array}{l}\text { (Millie's Meander) } \\
\text { TAPS A/G; Parallel }\end{array}$ & $\mathrm{CN} ; \mathrm{GR}$ & jafemrSSSSSSSSSSSSSSnode & $\begin{array}{l}1096222 \\
1096282 \\
1412+00\end{array}$ & $\begin{array}{l}\mathrm{F} \\
32 \mathrm{~N} \\
10 \mathrm{~W} \\
04 \\
\end{array}$ & $\begin{array}{l}\mathrm{AB} \\
\mathrm{F}\end{array}$ \\
\hline $\begin{array}{c}207.76 \\
103\end{array}$ & $\begin{array}{l}\text { (Way Back CK) } \\
\text { TAPS B/G; LWC }\end{array}$ & GR? & jafemrSSSSSSSSSSSSSSnode & $\begin{array}{l}1096975 \\
1406+00\end{array}$ & $\begin{array}{l}\mathrm{F} \\
32 \mathrm{~N} \\
10 \mathrm{~W} \\
09\end{array}$ & $\begin{array}{ll}\mathrm{AB} & \mathrm{E} \\
\mathrm{F} & \mathrm{I}\end{array}$ \\
\hline
\end{tabular}


USDOI BLM PMO OPEN FILE REPORT - TAPS FISH STREAMS

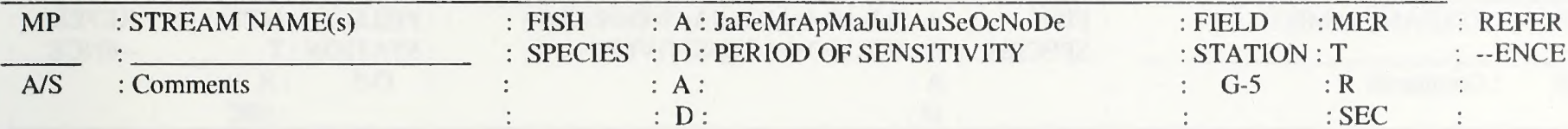

\begin{tabular}{|c|c|c|c|c|c|c|}
\hline $\begin{array}{c}208.01 \\
208.45 \\
103\end{array}$ & $\begin{array}{l}\text { Middle Fork KOYUKUK } \\
\text { RIVER } \\
\text { TAPS A/G; BLOCKPOINT }\end{array}$ & $\begin{array}{l}\text { BB?CN; } \\
\text { DS?DV; } \\
\text { GR;LS; } \\
\text { NP?RW }\end{array}$ & $\begin{array}{l}\text { CCCCCCCCCCCCCCCCCCCCCCCC } \\
\mathrm{E}_{1} \\
\mathrm{~S} \\
\vdots\end{array}$ & $\begin{array}{l}1098300 \\
1100600 \\
1384+47 \\
1361+45\end{array}$ & $\begin{array}{l}\mathrm{F} \\
32 \\
10 \mathrm{~W} \\
09\end{array}$ & $\begin{array}{ll}\mathrm{AB} & \mathrm{E} \\
\mathrm{F} & \end{array}$ \\
\hline $\begin{array}{c}209.02 \\
103\end{array}$ & $\begin{array}{l}\text { (North Fork, Sukakpak CK) } \\
\text { TAPS A/G; LWC }\end{array}$ & $\mathrm{CD} ; \mathrm{GR}$ & jafemrSSSSSSSSSSSSSSnode & $\begin{array}{l}1103583 \\
1103643 \\
1332+30\end{array}$ & $\begin{array}{l}\mathrm{F} \\
32 \mathrm{~N} \\
10 \mathrm{~W} \\
16\end{array}$ & $\begin{array}{l}\mathrm{BC} \\
\mathrm{F}\end{array}$ \\
\hline $\begin{array}{c}209.54 \\
103\end{array}$ & $\begin{array}{l}\text { (West Fork, Sukapak CK or } \\
\text { Pamplin's Potholes) } \\
\text { TAPS A/G; LWC }\end{array}$ & $\begin{array}{l}\text { CN;DV; } \\
\text { GR }\end{array}$ & jafemrCCCCCCSSSSCCCCnode & $\begin{array}{l}1106337 \\
1106407 \\
1305+50\end{array}$ & $\begin{array}{l}\mathrm{F} \\
32 \mathrm{~N} \\
10 \mathrm{~W} \\
16\end{array}$ & $\begin{array}{l}\text { A B C D } \\
\text { F }\end{array}$ \\
\hline $\begin{array}{c}210.03 \\
103\end{array}$ & $\begin{array}{l}\text { (East Fork, Sukakpak CK) } \\
\text { TAPS A/G; PARALLEL ONLY } \\
\text { TAPS DOES NOT CROSS }\end{array}$ & GR & jafemrSSSSSSSSSSSSSSnode & $\begin{array}{l}1108862 \\
1109102 \\
1276+77\end{array}$ & $\begin{array}{l}\mathrm{F} \\
32 \mathrm{~N} \\
10 \mathrm{~W} \\
21\end{array}$ & $\begin{array}{l}\mathrm{ABC} \\
\mathrm{F}\end{array}$ \\
\hline $\begin{array}{c}210.22 \\
103\end{array}$ & $\begin{array}{l}\text { (Marsh CK) } \\
\text { TAPS A/G; CMP }\end{array}$ & $\mathrm{CN} ; \mathrm{GR}$ & jafemrCCCCCCSSSSCCCCnode & $\begin{array}{l}1109943 \\
1110003 \\
1268+96\end{array}$ & $\begin{array}{l}\mathrm{F} \\
32 \mathrm{~N} \\
10 \mathrm{~W} \\
21\end{array}$ & $\begin{array}{l}\text { A B C } \\
F\end{array}$ \\
\hline $\begin{array}{c}210.43 \\
103\end{array}$ & $\begin{array}{l}\text { (Marsh CK) } \\
\text { TAPS A/G; LWC } \\
\text { Fish parallel to ROW } \\
\end{array}$ & GR;LS & jafemrCCCCCCSSSSCCCCnode & & $\begin{array}{l} \\
32 \mathrm{~N} \\
10 \mathrm{~W} \\
21\end{array}$ & $\mathbf{J}$ \\
\hline $\begin{array}{c}210.57 \\
103\end{array}$ & $\begin{array}{l}\text { (Marsh CK) } \\
\text { TAPS A/G; LWC }\end{array}$ & $\begin{array}{l}\text { CN;GR } \\
\text { LS }\end{array}$ & jafemrCCCCCCSSSSCCCCnode & $\begin{array}{l}1111792 \\
1111862 \\
1258+35\end{array}$ & $\begin{array}{l}\text { F } \\
32 \mathrm{~N} \\
10 \mathrm{~W} \\
21\end{array}$ & $\begin{array}{ll}\mathrm{ABC}^{2} & \\
\mathrm{~F} & \mathrm{~J}\end{array}$ \\
\hline $\begin{array}{c}210.94 \\
103\end{array}$ & $\begin{array}{l}\text { (Marsh CK) } \\
\text { TAPS B/G; LWC }\end{array}$ & $\mathrm{CN} ; \mathrm{GR}$ & jafemrCCCCCCSSSSCCCCnode & $\begin{array}{l}1113750 \\
1250+00\end{array}$ & $\begin{array}{l}\mathrm{F} \\
32 \mathrm{~N} \\
10 \mathrm{~W} \\
20\end{array}$ & $\begin{array}{l}A B C \\
F\end{array}$ \\
\hline $\begin{array}{c}210.94 \\
211.41 \\
103 \\
\end{array}$ & $\begin{array}{l}\text { Middle Fork, KOYUKUK } \\
\text { RIVER } \\
\text { TAPS B/G; BLOCKPOINT }\end{array}$ & $\begin{array}{l}\text { BB? CN } \\
\text { DS?DV;G } \\
\text { R;LS;NP? } \\
\text { RW }\end{array}$ & \begin{tabular}{l:l}
$Y$ & SSSSSSCCCCCCSSSSCCCCSSSS \\
\hdashline & \\
$S$ &
\end{tabular} & $\begin{array}{l}1113750 \\
1116250 \\
1237+55 \\
1207+63\end{array}$ & $\begin{array}{l}\mathrm{F} \\
32 \mathrm{~N} \\
10 \mathrm{~W} \\
20 \& 29 \\
\end{array}$ & $\begin{array}{ll}\text { A B } & \text { E } \\
F & \end{array}$ \\
\hline $\begin{array}{c}211.49 \\
102\end{array}$ & $\begin{array}{l}\text { (One-0-Two-North CK) } \\
\text { TAPS A/G; LWC }\end{array}$ & $\overline{G R}$ & & $\begin{array}{l}1116641 \\
1116696 \\
1202+40\end{array}$ & $\begin{array}{l}\mathrm{F} \\
32 \mathrm{~N} \\
10 \mathrm{~W} \\
29\end{array}$ & B \\
\hline $\begin{array}{c}212.79 \\
102\end{array}$ & $\begin{array}{l}\text { (Spoiled CK) } \\
\text { TAPS A/G; LWC }\end{array}$ & GR? & jafemrSSSSSSSSSSSSSSnode & $\begin{array}{l}1123521 \\
1123580\end{array}$ & $\begin{array}{l}\mathrm{F} \\
32 \mathrm{~N} \\
10 \mathrm{~W} \\
32\end{array}$ & I \\
\hline $\begin{array}{c}212.89 \\
102\end{array}$ & $\begin{array}{l}\text { (Koyukuk Slough) } \\
\text { TAPS A/G; LWC }\end{array}$ & GR? & jafemrCCCCCCSSauseocnode & $\begin{array}{c}1124001 \\
1124071 \\
?\end{array}$ & $\begin{array}{l}\mathrm{F} \\
32 \mathrm{~N} \\
10 \mathrm{~W} \\
31\end{array}$ & ${ }_{1}^{E}$ \\
\hline $\begin{array}{c}213.03 \\
102\end{array}$ & $\begin{array}{l}\text { (Valve Site CK) } \\
\text { TAPS A/G; CMP }\end{array}$ & GR? & & $\begin{array}{l}1124772 \\
1124831 \\
1121+05\end{array}$ & $\begin{array}{l}\mathrm{F} \\
32 \mathrm{~N} \\
10 \mathrm{~W} \\
31\end{array}$ & $F^{B}$ \\
\hline $\begin{array}{c}215.31 \\
102\end{array}$ & $\begin{array}{l}\text { LINDA CREEK } \\
\text { TAPS A/G; BLOCKPOINT }\end{array}$ & $\mathrm{CN} ; \mathrm{GR}$ & jafemrSSSSSSSSSSSSSSnode & $\begin{array}{l}1136782 \\
1136900 \\
1001+18\end{array}$ & $\begin{array}{l}\mathrm{F} \\
31 \mathrm{~N} \\
10 \mathrm{~W}\end{array}$ & $\begin{array}{l}\text { A B } \\
\text { F G }\end{array}$ \\
\hline
\end{tabular}


USDOI BLM PMO OPEN FILE REPORT - TAPS FISH STREAMS

\begin{tabular}{|c|c|c|c|c|c|c|}
\hline MP & $\begin{array}{l}\text { : STREAM NAME(s) } \\
:\end{array}$ & $\begin{array}{l}: \text { FISH } \\
: \text { SPECIES }\end{array}$ & $\begin{array}{l}\text { : A : JaFeMrApMaJuJIAuSeOcNoDe } \\
: \text { D : PERIOD OF SENSITIVITY }\end{array}$ & $\begin{array}{l}\text { : FIELD } \\
: \text { STATION }\end{array}$ & $\begin{array}{l}: \mathrm{MER} \\
: \mathrm{T}\end{array}$ & $\begin{array}{l}\text { : REFER } \\
: \text {--ENCE }\end{array}$ \\
\hline A/S & : Comments & $\begin{array}{l}: \\
: \\
\end{array}$ & $\begin{array}{l}: \mathrm{A}: \\
: \mathrm{D}: \\
\end{array}$ & $: \quad$ G-5 & $\begin{array}{l}: \text { R } \\
: \text { SEC }\end{array}$ & \\
\hline $\begin{array}{r}215.81 \\
102\end{array}$ & $\begin{array}{l}\text { GOLD CREEK } \\
\text { TAPS B/G; SUMMER } \\
\text { BLOCKPOINT }\end{array}$ & $\begin{array}{l}\mathrm{CN} ; \mathrm{DV} ? \\
\mathrm{GR} ; \mathrm{RW} ?\end{array}$ & $\begin{array}{c:cccc}\text { jafemrCCCCCCSSSCCCCnode } \\
\vdots \\
\end{array}$ & $\begin{array}{r}1139410 \\
1139550 \\
976+00 \\
\end{array}$ & $\begin{array}{ll}\mathrm{F} \\
31 \mathrm{~N} \\
10 \mathrm{~W} \\
18 \\
\end{array}$ & $\begin{array}{l:l}\text { A B } \\
\text { F G } \\
\end{array}$ \\
\hline $\begin{array}{c}216.30 \\
102\end{array}$ & $\begin{array}{l}\text { (Cushing CK) } \\
\text { TAPS AVG; LWC }\end{array}$ & GR? & 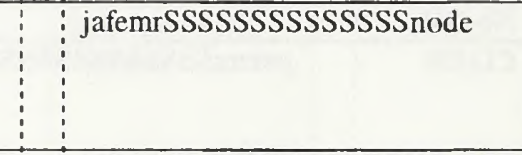 & $\begin{array}{r}1142019 \\
1142074 \\
948+66 \\
\end{array}$ & $\begin{array}{l:l}\mathrm{F} \\
31 \mathrm{~N} \\
10 \mathrm{~W} \\
18 \\
\end{array}$ & A B \\
\hline $\begin{array}{c}216.59 \\
102\end{array}$ & $\begin{array}{l}\text { SHEEP CREEK } \\
\text { TAPS A/G; LWC }\end{array}$ & $\mathrm{CN} ; \mathrm{GR}$ & : jafemrCCCCCCSSSSCCCCnode & $\begin{array}{r}1143574 \\
1143369 \\
933+01 \\
\end{array}$ & $\begin{array}{l:l}\mathrm{F} \\
31 \mathrm{~N} \\
10 \mathrm{~W} \\
19 \\
\end{array}$ & $\begin{array}{l}\mathrm{A} \mathrm{B} \\
\mathrm{F}\end{array}$ \\
\hline $\begin{array}{r}217.07 \\
102\end{array}$ & $\begin{array}{l}\text { WOLF PUP CREEK } \\
\text { TAPS AVG; CMP }\end{array}$ & $\mathrm{CN}$ & jafemrSSSSSSSSSSSSSSnode & $\begin{array}{r}1146100 \\
1146160 \\
908+00\end{array}$ & $\begin{array}{l}F \\
31 \mathrm{~N} \\
10 \mathrm{~W} \\
19\end{array}$ & F \\
\hline $\begin{array}{c}217.09 \\
102\end{array}$ & $\begin{array}{l}\text { WOLF PUP CREEK } \\
\text { TAPS AVG; CMP }\end{array}$ & $\mathrm{CN} ; \mathrm{GR}$ ? & jafemrSSSSSSSSSSSSSSnode & $\begin{array}{r}1146220 \\
1146269 \\
906+78 \\
\end{array}$ & $\begin{array}{l:}F \\
31 N \\
10 W \\
19 \\
\end{array}$ & $\begin{array}{l:}\text { A B } \\
\text { F }\end{array}$ \\
\hline $\begin{array}{c}217.49 \\
101\end{array}$ & $\begin{array}{l}\text { NUGGET CREEK } \\
\text { TAPS AVG; LWC }\end{array}$ & $\begin{array}{l}\mathrm{CN} ; \mathrm{DV} \\
\mathrm{GR} ; \mathrm{RW} ?\end{array}$ & $\begin{array}{l:l}\text { jafemrSSSSSSSSSSSSSSnode } \\
& \end{array}$ & $\begin{array}{r}1148336 \\
1148391 \\
886+52\end{array}$ & $\begin{array}{l}\mathrm{F} \\
31 \mathrm{~N} \\
10 \mathrm{~W} \\
19 \\
\end{array}$ & $\begin{array}{ll}\mathrm{AB} \\
\mathrm{FG}\end{array}$ \\
\hline $\begin{array}{c}218.00 \\
101\end{array}$ & $\begin{array}{l}\text { (Alignment Slough) } \\
\text { TAPS A/G; CMP }\end{array}$ & CD? GR & jafemrCCCCCCSSSSCCCCnode & $\begin{array}{r}1150992 \\
1151052 \\
859+99\end{array}$ & $\begin{array}{l}F \\
31 N \\
11 W \\
25 \\
\end{array}$ & $\begin{array}{l}\text { A B } \\
\text { F }\end{array}$ \\
\hline $\begin{array}{c}218.08 \\
101\end{array}$ & $\begin{array}{l}\text { (Alignment Slough) } \\
\text { TAPS A/G; CMP }\end{array}$ & CD? GR & jafemrCCCCCCSSSSCCCCnode & $\begin{array}{c}1151409 \\
1151469 \\
855+94\end{array}$ & $\begin{array}{l}F \\
31 N \\
11 W \\
25 \\
\end{array}$ & $\begin{array}{l}\text { A B } \\
\text { F }\end{array}$ \\
\hline $\begin{array}{c}218.14 \\
101\end{array}$ & $\begin{array}{l}\text { (Alignment Slough) } \\
\text { TAPS A/G; LWC }\end{array}$ & CD? GR? & jafemrCCCCCCSSSSCCCCnode & $\begin{array}{r}1151760 \\
1151835 \\
848+93\end{array}$ & $\begin{array}{l}F \\
31 N \\
11 W \\
25 \\
\end{array}$ & $\begin{array}{ll}\mathrm{AB} & \mathrm{E} \\
\mathrm{F} & \mathrm{I}\end{array}$ \\
\hline $\begin{array}{c}218.20 \\
101\end{array}$ & $\begin{array}{l}\text { (Alignment Slough) } \\
\text { TAPS A/G; CMP }\end{array}$ & CD? GR & jafemrCCCCCCSSSSCCCCnode & $\begin{array}{r}1152070 \\
1152139 \\
845+28\end{array}$ & $\begin{array}{l:}F \\
31 N \\
11 W \\
25 \\
\end{array}$ & $\begin{array}{l}\text { A B } \\
\text { F }\end{array}$ \\
\hline $\begin{array}{c}218.28 \\
101\end{array}$ & $\begin{array}{l}\text { (Alignment Slough) } \\
\text { TAPS AVG; LWC }\end{array}$ & CD? GR? & jafemrCCCCCCSSSSCCCCnode & $\begin{array}{r}1152492 \\
1152556 \\
843+20\end{array}$ & \begin{tabular}{|l}
$\mathrm{F}$ \\
$31 \mathrm{~N}$ \\
$11 \mathrm{~W}$ \\
25 \\
\end{tabular} & $\begin{array}{lll:}A B & E\end{array}$ \\
\hline $\begin{array}{c}218.35 \\
101\end{array}$ & $\begin{array}{l}\text { (Alignment Slough) } \\
\text { TAPS A/G; CMP }\end{array}$ & CD? GR & jafemrCCCCCCSSSSCCCCnode & $\begin{array}{r}1152847 \\
1152902 \\
840+20 \\
\end{array}$ & \begin{tabular}{|l}
$F$ \\
$31 N$ \\
$11 W$ \\
25 \\
\end{tabular} & $\begin{array}{l}\text { A B } \\
\text { F } \\
\\
\end{array}$ \\
\hline $\begin{array}{r}218.45 \\
101\end{array}$ & $\begin{array}{l}\text { (Alignment Slough) } \\
\text { TAPS A/G; CMP }\end{array}$ & CD? GR & jafemrCCCCCCSSSSCCCCnode & $\begin{array}{r}1153361 \\
1153434 \\
835+63 \\
\end{array}$ & $\begin{array}{l:l}\mathrm{F} \\
31 \mathrm{~N} \\
11 \mathrm{~W} \\
25 \\
\end{array}$ & $\begin{array}{ll}A \text { B } \\
F \\
\end{array}$ \\
\hline 219.04 & $\begin{array}{l}\text { OVER CREEK (Complex) } \\
\text { TAPS AVG; CMP }\end{array}$ & $\mathrm{BB} ; \mathrm{GR}$ & jafemrSSSSSSSSSSSSSSnode & $\begin{array}{r}1156490 \\
1156552 \\
805+39 \\
\end{array}$ & $\begin{array}{ll}F \\
31 N \\
11 W \\
26 \\
\end{array}$ & \begin{tabular}{|l|l}
$\mathrm{AB}$ & $\mathrm{E}$ \\
$\mathrm{F}$ & \\
& \\
\end{tabular} \\
\hline $\begin{array}{c}219.08 \\
101\end{array}$ & $\begin{array}{l}\text { Tributary OVER CREEK } \\
\text { TAPS A/G; LWC } \\
\end{array}$ & BB; GR & jafemrSSSSSSSSSSSSSSnode & $\begin{array}{r}1156732 \\
1156793 \\
803+12\end{array}$ & $\begin{array}{ll}F \\
31 N \\
11 W \\
26 \\
\end{array}$ & $\begin{array}{ll}\text { A B B } \\
\text { F } \\
\\
\end{array}$ \\
\hline
\end{tabular}


USDOI BLM PMO OPEN FILE REPORT - TAPS FISH STREAMS

\begin{tabular}{llll}
\hline $\mathrm{MP}$ & $:$ STREAM NAME(s) & $:$ FISH & $:$ A : JaFeMrApMaJuJlAuSeOcNoDe \\
& $:$ & $:$ SPECIES & $:$ D : PERIOD OF SENSITIVITY \\
$\mathrm{A} / \mathrm{S}$ & $:$ Comments & $:$ & $:$ A : \\
& & $:$ & $:$ D :
\end{tabular}

219.13 OVER CREEK (Complex)

101 TAPS A/G; CMP

219.20 OVER CREEK (Complex or

Rainbow CK)

101 TAPS A/G; CMP

219.52 (Richardson Slough or Coon Gulch?)

$101 \quad$ TAPS A/G; LWC

$219.60 \quad$ (Richardson Slough)

$101 \quad$ TAPS A/G; LWC

\begin{tabular}{|l|l}
\hline 221.79 & (One-0-One CK)
\end{tabular}

101 TAPS A/G; LWC

\begin{tabular}{|l|l}
\hline 221.96 & Middle Fork, KOYUKUK
\end{tabular}

222.07 RIVER

101 TAPS A/G; BLOCKPOINT

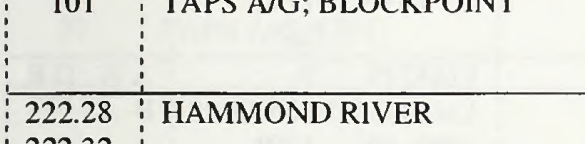

222,32

101 TAPS A/G; BLOCKPOINT

\begin{tabular}{|l|l}
\hline 223.19 & (Confederate Gulch or Fry CK)
\end{tabular}

$\begin{array}{l:l}100 & \text { TAPS A/G; LWC }\end{array}$

\begin{tabular}{|l|l} 
& \\
223.67 & (Union Gulch)
\end{tabular}

100 TAPS A/G; LWC

224.23 (Union Gulch CK)

$\begin{array}{llll} & & \\ 100 & \text { TAPS A/G; LWC }\end{array}$

\begin{tabular}{|l:l} 
& \\
224.55 & (Union Gulch CK)
\end{tabular}

\begin{tabular}{|c|l}
\hline 100 & TAPS A/G; BLOCKPOINT \\
\hline 224.62 & Middle Fork, KOYUKUK \\
222.94 & RIVER \\
101 & TAPS A/G; BLOCKPOINT \\
\hline
\end{tabular}

\begin{tabular}{|l|l}
\hline 225.76 & MINNIE CREEK
\end{tabular}

100 TAPS A/G; BLOCKPOINT

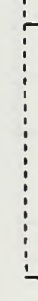

BB; GR jafemrSSSSSSSSSSSSSSnode

$\mathrm{CN}$

GR; LS? $:$ jafemrSSSSSSSSSSSSSSnode

$\mathrm{RW}$ ?

$\vdots$
$\vdots$

CN? GR; $\quad$ jafemrSSSSSSSSSSSSSSnode

LS; RW

CN; GR; $\quad$ jafemrCCCCCCSSSSCCCnode

LS?

BB? CN $\quad \mathrm{CCCCCCCCCCCSSCCCCCCCCCC}$

CS? DS; $\mathrm{E}$

DV; GR; $\quad$ S:

KS?LS;

NP?RW

CN;DV; $\quad \mathrm{Y}_{1}^{\text {jafemrSSSSSSSSSSSSSSnode }}$

GR;KS

E:

WF; LS S:

DS

(1)

jafemrCCCCCCSSSSCCCCnode

\begin{tabular}{ll:l}
\hline CN;GR & jafemrSSSSSSSSSSSSSSnode
\end{tabular}

LS?RW?

jafemr $\underline{\underline{S S S S S S S S S S S S S S n o d e ~}}$

CN;GR; $\quad \vdots$ jafemrCCCCCCSSSSCCCCnode

LS?RW

CN;GR; jafemrCCCCCCSSSSCCCCnode

$\mathrm{LS} ; \mathrm{RW}$

CN;DS; $\quad Y$ CCCCCCCCCCCCSSCCCCCCCCCC

DV;GR; $\quad \mathrm{E}^{\prime}$

KS;LS; $\quad$ S:

NP?RW

$\mathrm{BB} ; \mathrm{CN}$;

DV;GR;

jafemrSSSSSSSSSSSSSSnode

LS;RW

$\mathrm{CN} ; \mathrm{GR}$

jafemrSSSSSSSSSSSSSSnode

$100 \quad$ TAPS B/G; CMP

07/30/03 PAGE 19 of 41

$\begin{array}{lll}: \text { FIELD }: \text { MER } & \text { : REFER } \\ : \text { STATION : T } & :-- \text { ENCE } \\ : \text { G-5 }: \text { R } & :\end{array}$

: $R$

\begin{tabular}{r|l:lr:}
1156963 & $\mathrm{~F}$ & $\mathrm{~A}$ B & $\mathrm{E}$ \\
1157033 & $31 \mathrm{~N}$ & $\mathrm{~F}$ & \\
$802+12$ & $11 \mathrm{~W}$ & & \\
& 35 & & \\
\hline 1157333 & $\mathrm{~F}$ & A B & $\mathrm{E}$ \\
\hline
\end{tabular}

$1157403 \quad 31 \mathrm{~N}$

$\begin{array}{ll:}796+36 & 11 \mathrm{~W}\end{array}$

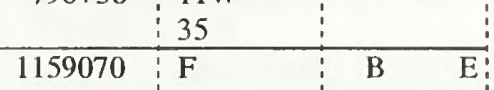

$\begin{array}{r:r}1159135 & 31 \mathrm{~N} \\ 781+90 & 11 \mathrm{~W}\end{array}$

$\mathrm{F}$

$781+90 \quad 11 \mathrm{~W}$

\begin{tabular}{l|l|ll} 
& 35 & & \\
\hline 1159430 & $F$ & A B & E
\end{tabular}

$778+00 \quad 11 \mathrm{~W}$

$\mathrm{F}$

\begin{tabular}{l|l|l} 
& 35 & \\
\hline 1171039 & $\mathrm{~F}$ & A B $\quad \mathrm{E}$
\end{tabular}

$1171101 \quad 31 \mathrm{~N}$

F

$664+00 \quad 11 \mathrm{~W}$

$\begin{array}{l:l} & 33 \\ 1171932 & \mathrm{~F}\end{array}$

$\begin{array}{lll}1172525 & 31 \mathrm{~N}\end{array}$

$651+50 \quad 11 \mathrm{~W}$

33

1173644

$\begin{array}{lll}1173864 & 31 \mathrm{~N}\end{array}$

$\begin{array}{lll}635+50 & 11 \mathrm{~W}\end{array}$

\begin{tabular}{l:l} 
& $32 \& 33$ \\
\hline 1178399 & $\mathrm{~F}$
\end{tabular}

$\begin{array}{l:l}1178470 & 30 \mathrm{~N}\end{array}$

$\begin{array}{lll}590+75 & 11 \mathrm{~W}\end{array}$

05

1180957

1180957

\begin{tabular}{l|l}
1180957 & $\mathrm{~F}$ \\
\hline 1181027 & $30 \mathrm{~N}$
\end{tabular}

A B E:

$\mathrm{F}$

A B E

F J

B

F 1

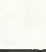

IJ

$\mathrm{F} \quad 1 \mathrm{~J}$

$\begin{array}{l:ll}565+10 & 11 \mathrm{~W}\end{array}$ 
USDOI BLM PMO OPEN FILE REPORT - TAPS FISH STREAMS 07/30/03 PAGE 20 of 41

\begin{tabular}{|c|c|c|c|c|c|c|}
\hline MP & STREAM NAME(s) & $\begin{array}{l}\text { FISH } \\
\text { SPECIES }\end{array}$ & $\begin{array}{l}\text { A : JaFeMrApMaJuJlAuSeOcNoDe } \\
\text { D : PERIOD OF SENSITIVITY }\end{array}$ & : FIELD & MER & $\begin{array}{l}\text { REFER } \\
\text {--ENCE }\end{array}$ \\
\hline $\mathrm{A} / \mathrm{S}$ & : Comments & & $\begin{array}{l}: \text { A : } \\
: \text { D : }\end{array}$ & : G-5 & $\begin{array}{l}: \mathrm{R} \\
: \mathrm{SEC}\end{array}$ & \\
\hline $\begin{array}{c}232.78 \\
99\end{array}$ & $\begin{array}{l}\text { (Pence's Pond) } \\
\text { TAPS B/G; CMP }\end{array}$ & $\mathrm{CD}$ ?GR & jafemrSSSSSSSSSSSSSSnode & $\begin{array}{r}1229086 \\
85+80\end{array}$ & $\begin{array}{l}\mathrm{F} \\
29 \mathrm{~N} \\
12 \mathrm{~W} \\
23\end{array}$ & $\begin{array}{l}\text { A B } \\
\text { F }\end{array}$ \\
\hline $\begin{array}{c}233.26 \\
99\end{array}$ & $\begin{array}{l}\text { MARION CREEK } \\
\text { TAPS B/G; BLOCKPOINT }\end{array}$ & $\begin{array}{l}\text { CN;DV; } \\
\text { GR;KS;R } \\
\text { W }\end{array}$ & jafemrSSSSSSSSSSSSSSnode & $\begin{array}{r}1231600 \\
61+85 \\
59+85\end{array}$ & $\begin{array}{l}\mathrm{F} \\
29 \mathrm{~N} \\
12 \mathrm{~W} \\
23\end{array}$ & $\begin{array}{l}\text { A B } \\
\text { F G }\end{array}$ \\
\hline $\begin{array}{c}233.38 \\
99\end{array}$ & $\begin{array}{l}\text { (Sharon } \mathrm{CK} \text { ) } \\
\text { TAPS B/G; CMP }\end{array}$ & $\begin{array}{l}\text { BB?CN? } \\
\text { GR?LS? } \\
\text { RW? }\end{array}$ & jafemrSSSSSSSSSSSSSSnode & $\begin{array}{r}1232225 \\
55+00\end{array}$ & $\begin{array}{l}\mathrm{F} \\
29 \mathrm{~N} \\
12 \mathrm{~W} \\
26\end{array}$ & $\begin{array}{l}\mathrm{AB} \\
\mathrm{F}\end{array}$ \\
\hline $\begin{array}{c}233.54 \\
99\end{array}$ & $\begin{array}{l}\text { (Sharon } \mathrm{CK} \text { ) } \\
\text { TAPS B/G; CMP }\end{array}$ & $\begin{array}{l}\text { BB?CN? } \\
\text { GR?LS? } \\
\text { RW? }\end{array}$ & jafemrSSSSSSSSSSSSSSnode & $\begin{array}{r}1233075 \\
45+43\end{array}$ & $\begin{array}{l}\mathrm{F} \\
29 \mathrm{~N} \\
12 \mathrm{~W} \\
26 \\
\end{array}$ & $\begin{array}{l}\mathrm{AB} \\
\mathrm{F}\end{array}$ \\
\hline $\begin{array}{c}234.24 \\
99\end{array}$ & $\begin{array}{l}\text { (Mary Angel CK) } \\
\text { TAPS B/G; CMP }\end{array}$ & $\begin{array}{l}\mathrm{BB} ; \mathrm{CN} ; \\
\mathrm{GR} ; \mathrm{LS} ; \\
\mathrm{WF}\end{array}$ & jafemrCCCCCCSSSSCCCCnode & $\begin{array}{r}1236800 \\
8+47\end{array}$ & $\begin{array}{l}\mathrm{F} \\
29 \mathrm{~N} \\
12 \mathrm{~W} \\
26 \\
\end{array}$ & $\begin{array}{l}\text { A B } \\
\text { F }\end{array}$ \\
\hline $\begin{array}{c}234.31 \\
98\end{array}$ & $\begin{array}{l}\text { (South Fork, Mary Angel CK) } \\
\text { TAPS B/G; CMP }\end{array}$ & $\mathrm{CN} ; \mathrm{GR}$ & jafemrSSSSSSSSSSSSSSnode & $\begin{array}{r}1237150 \\
4+30\end{array}$ & $\begin{array}{l}\mathrm{F} \\
29 \mathrm{~N} \\
12 \mathrm{~W} \\
26 \\
\end{array}$ & $\begin{array}{l}\text { A B } \\
\text { F }\end{array}$ \\
\hline $\begin{array}{c}235.49 \\
98\end{array}$ & $\begin{array}{l}\text { (Texas Slough) } \\
\text { TAPS B/G;; PARALLEL ONLY; } \\
\text { TAPS DOES NOT CROSS }\end{array}$ & $\begin{array}{l}\mathrm{CN} ; \mathrm{GR} ; \\
\mathrm{KS}\end{array}$ & jafemrSSSSSSSSSSSSSSnode & $\begin{array}{l}1243275 \\
1243475 \\
1089+00 \\
1087+00 \\
\end{array}$ & $\begin{array}{l}\mathrm{F} \\
28 \mathrm{~N} \\
12 \mathrm{~W} \\
03 \\
\end{array}$ & $\begin{array}{l}\text { A B D E } \\
F\end{array}$ \\
\hline $\begin{array}{c}235.62 \\
98\end{array}$ & $\begin{array}{l}\text { (1079 Slough) } \\
\text { TAPS B/G; CMP }\end{array}$ & $\mathrm{CN} ; \mathrm{GR}$ & jafemrCCCCCCSSSSCCCCnode & $\begin{array}{l}1244062 \\
1079+50\end{array}$ & $\begin{array}{l}\mathrm{F} \\
28 \mathrm{~N} \\
12 \mathrm{~W} \\
03 \\
\end{array}$ & $\begin{array}{l}\text { A B } \\
F\end{array}$ \\
\hline $\begin{array}{c}236.07 \\
98\end{array}$ & $\begin{array}{l}\text { (Oregano CK) } \\
\text { TAPS B/G; LWC }\end{array}$ & $\begin{array}{l}\text { CN?DV; } \\
\text { GR }\end{array}$ & jafemrSSSSSSSSSSSSSSnode & $\begin{array}{l}1246450 \\
1057+00\end{array}$ & $\begin{array}{l}\mathrm{F} \\
28 \mathrm{~N} \\
12 \mathrm{~W} \\
03 \\
\end{array}$ & $\begin{array}{l}\text { A B } \\
\text { F }\end{array}$ \\
\hline $\begin{array}{c}236.17 \\
98\end{array}$ & $\begin{array}{l}\text { (Equisetum CK) } \\
\text { TAPS B/G; CMP }\end{array}$ & GR & jafemrSSSSSSSSSSSSSSnode & $\begin{array}{l}1247000 \\
1051+80\end{array}$ & $\begin{array}{l}\mathrm{F} \\
28 \mathrm{~N} \\
12 \mathrm{~W} \\
03 \\
\end{array}$ & $\begin{array}{l}\text { A B } \\
\text { F }\end{array}$ \\
\hline 236.45 & $\begin{array}{l}\text { CLARA CREEK } \\
\text { TAPS A/G; CMP }\end{array}$ & GR? & jafemrSSSSSSSSSSSSSSnode & $\begin{array}{l}1248425 \\
1248490 \\
1036+20\end{array}$ & $\begin{array}{l}\mathrm{F} \\
28 \mathrm{~N} \\
12 \mathrm{~W} \\
10 \\
\end{array}$ & $\mathrm{~F}$ \\
\hline $\begin{array}{c}236.49 \\
98\end{array}$ & $\begin{array}{l}\text { CLARA CREEK } \\
\text { TAPS A/G; CMP }\end{array}$ & $\begin{array}{l}\text { CN?GR; } \\
\text { RW? }\end{array}$ & jafemrSSSSSSSSSSSSSSnode & $\begin{array}{l}1248648 \\
1248715 \\
1033+06\end{array}$ & $\begin{array}{l}\mathrm{F} \\
28 \mathrm{~N} \\
12 \mathrm{~W} \\
10 \\
\end{array}$ & $\begin{array}{l}\mathrm{AB} \\
\mathrm{F}\end{array}$ \\
\hline $\begin{array}{c}236.76 \\
98\end{array}$ & $\begin{array}{l}\text { CLARA CREEK Overflow } \\
\text { TAPS A/G; CMP }\end{array}$ & $\begin{array}{l}\text { CN?GR; } \\
\text { RW }\end{array}$ & jafemrSSSSSSSSSSSSSSnode & $\begin{array}{l}1250077 \\
1250137 \\
1019+50\end{array}$ & $\begin{array}{l}\mathrm{F} \\
28 \mathrm{~N} \\
12 \mathrm{~W} \\
10 \\
\end{array}$ & $\begin{array}{l}\mathrm{AB} \\
\mathrm{F}\end{array}$ \\
\hline $\begin{array}{c}236.83 \\
98\end{array}$ & $\begin{array}{l}\text { South Fork, CLARA CK } \\
\text { TAPS A/G; CMP }\end{array}$ & $\begin{array}{l}\text { CN;DV? } \\
\text { GR;RW }\end{array}$ & jafemrSSSSSSSSSSSSSSnode & $\begin{array}{l}1250437 \\
1250497 \\
1015+50\end{array}$ & $\begin{array}{l}\mathrm{F} \\
28 \mathrm{~N} \\
12 \mathrm{~W} \\
10\end{array}$ & $\begin{array}{l}\mathrm{AB} \\
\mathrm{F}\end{array}$ \\
\hline
\end{tabular}


USDOI BLM PMO OPEN FILE REPORT - TAPS FISH STREAMS

$\begin{array}{llll}\mathrm{MP} & : \text { STREAM NAME(s) } & : \text { FISH } & : \text { A }: \text { JaFeMrApMaJuJIAuSeOcNoDe } \\ & : \text { SPECIES } & : \text { D : PERIOD OF SENSITIVITY } \\ \mathrm{A} / \mathrm{S} & : \text { Comments } & : & : \text { A : } \\ & & : & : \mathrm{D}:\end{array}$

\begin{tabular}{|c|c|c|c|c|c|c|}
\hline $\begin{array}{c}237.04 \\
98\end{array}$ & $\begin{array}{l}\text { (Calf CK) } \\
\text { TAPS A/G; BR1DGE }\end{array}$ & $\begin{array}{l}\text { CD?DV? } \\
\text { GR;RW? }\end{array}$ & jafemrSSSSSSSSSSSSSSnode & $\begin{array}{l}1251552 \\
1251612 \\
1004+30\end{array}$ & $\begin{array}{l}\mathrm{F} \\
28 \mathrm{~N} \\
12 \mathrm{~W} \\
10\end{array}$ & $\begin{array}{l}\mathrm{AB} \\
\mathrm{F}\end{array}$ \\
\hline $\begin{array}{c}237.57 \\
98\end{array}$ & $\begin{array}{l}\text { SLATE CREEK } \\
\text { TAPS B/G; BLOCKPOINT }\end{array}$ & $\begin{array}{l}\text { CN;DS; } \\
\text { DV;GR; } \\
\text { KS;RW }\end{array}$ & $\begin{array}{l}\mathrm{C}_{1} \\
\mathrm{E} \\
\mathrm{E} \\
\mathrm{S} \\
\vdots\end{array}$ & $\begin{array}{r}1254250 \\
1254450 \\
976+83\end{array}$ & $\begin{array}{l}\mathrm{F} \\
28 \mathrm{~N} \\
12 \mathrm{~W} \\
15\end{array}$ & $\begin{array}{l}\mathrm{AB} \\
\mathrm{FG}\end{array}$ \\
\hline $\begin{array}{c}239.26 \\
98\end{array}$ & $\begin{array}{l}\text { (Horseshoe Slough) } \\
\text { TAPS A/G; CMP }\end{array}$ & GR? & jafemrSSSSSSSSSSSSSSnode & $\begin{array}{r}1263276 \\
1263344 \\
888+00\end{array}$ & $\begin{array}{l}\mathrm{F} \\
28 \mathrm{~N} \\
12 \mathrm{~W} \\
20\end{array}$ & $\mathrm{AB}$ \\
\hline $\begin{array}{c}239.76 \\
98\end{array}$ & $\begin{array}{l}\text { (Spring Slough) } \\
\text { TAPS A/G; LWC }\end{array}$ & $\mathrm{CD} / \mathrm{GR}$ & jafemrCCCCCCSSSSCCCCnode & $\begin{array}{r}1265884 \\
1265947 \\
862+10\end{array}$ & $\begin{array}{l}\mathrm{F} \\
28 \mathrm{~N} \\
12 \mathrm{~W} \\
20 \\
\end{array}$ & $\begin{array}{ll}\text { A B } & \text { E } \\
\text { F } & \end{array}$ \\
\hline $\begin{array}{c}240.13 \\
97\end{array}$ & $\begin{array}{l}\text { (East Fork, Spring Slough) } \\
\text { TAPS A/G; CMP }\end{array}$ & GR & jafemrSSSSSSSSSSSSSSnode & $\begin{array}{r}1267880 \\
1267940 \\
842+00\end{array}$ & $\begin{array}{l}\mathrm{F} \\
28 \mathrm{~N} \\
12 \mathrm{~W} \\
29\end{array}$ & $\begin{array}{l}\text { A B } \\
F\end{array}$ \\
\hline $\begin{array}{c}240.26 \\
97\end{array}$ & $\begin{array}{l}\text { (East Fork, Spring Slough) } \\
\text { TAPS A/G; LWC }\end{array}$ & GR & jafemrSSSSSSSSSSSSSSnode & $\begin{array}{r}1268559 \\
1268619 \\
835+90\end{array}$ & $\begin{array}{l}\mathrm{F} \\
28 \mathrm{~N} \\
12 \mathrm{~W} \\
29 \\
\end{array}$ & $\begin{array}{l}\text { A B } \\
F\end{array}$ \\
\hline $\begin{array}{c}240.37 \\
97\end{array}$ & $\begin{array}{l}\text { (Spring Slough) } \\
\text { TAPS A/G; CMP }\end{array}$ & $\mathrm{CD} ? \mathrm{GR}$ & jafemrCCCCCCSSSSCCCCnode & $\begin{array}{r}1269132 \\
1269191 \\
829+50\end{array}$ & $\begin{array}{l}\mathrm{F} \\
28 \mathrm{~N} \\
12 \mathrm{~W} \\
29\end{array}$ & $\begin{array}{l}\text { A B } \\
F\end{array}$ \\
\hline $\begin{array}{c}240.66 \\
97\end{array}$ & $\begin{array}{l}\text { (South Fork, Spring Slough) } \\
\text { TAPS A/G; CMP }\end{array}$ & $\begin{array}{l}\text { CN?GR; } \\
\text { NP }\end{array}$ & jafemrSSSSSSSSSSSSSSnode & $\begin{array}{r}1270653 \\
1270713 \\
810+00\end{array}$ & $\begin{array}{l}\mathrm{F} \\
28 \mathrm{~N} \\
12 \mathrm{~W} \\
29\end{array}$ & $\begin{array}{l}\text { A B } \\
\mathrm{F}\end{array}$ \\
\hline $\begin{array}{c}240.79 \\
97\end{array}$ & $\begin{array}{l}\text { (Spring Slough) } \\
\text { TAPS A/G; CMP }\end{array}$ & $\mathrm{CN} ; \mathrm{GR}$ & jafemrCCCCCCSSSSCCCCnode & $\begin{array}{r}1271357 \\
1271411 \\
804+00\end{array}$ & $\begin{array}{l}\mathrm{F} \\
28 \mathrm{~N} \\
12 \mathrm{~W} \\
29\end{array}$ & $\begin{array}{l}\mathrm{AB} \\
\mathrm{F}\end{array}$ \\
\hline $\begin{array}{c}242.89 \\
97\end{array}$ & $\begin{array}{l}\text { ROSIE CREEK } \\
\text { TAPS B/G; LWC }\end{array}$ & $\begin{array}{l}\text { CN;DV? } \\
\text { GR;RW? } \\
\mathrm{KS}\end{array}$ & $\begin{array}{l} \\
\\
E_{1} \\
S\end{array}$ & $\begin{array}{l}1282475 \\
694+50\end{array}$ & $\begin{array}{l}\mathrm{F} \\
27 \mathrm{~N} \\
12 \mathrm{~W} \\
06\end{array}$ & $\begin{array}{ll}\text { A B } & \text { E } \\
F & \text { J }\end{array}$ \\
\hline $\begin{array}{c}243.41 \\
97\end{array}$ & $\begin{array}{l}\text { ROSIE CREEK } \\
\text { TAPS B/G; CMP }\end{array}$ & $\begin{array}{l}\mathrm{CN} ; \mathrm{DV} ; \\
\mathrm{GR} ; \mathrm{RW}\end{array}$ & SSSSSSCCCCCCSSSSCCCCSSSS & $\begin{array}{l}1285200 \\
666+00\end{array}$ & $\begin{array}{l}\mathrm{F} \\
27 \mathrm{~N} \\
13 \mathrm{~W} \\
01\end{array}$ & $\begin{array}{l}\mathrm{AB} \\
\mathrm{FG}\end{array}$ \\
\hline
\end{tabular}

\begin{tabular}{|c|c|c|c|c|c|c|}
\hline $\begin{array}{c}243.94 \\
97\end{array}$ & $\begin{array}{l}\text { (Mud CK) } \\
\text { TAPS B/G; CMP }\end{array}$ & $\mathrm{BB} ; \mathrm{GR}$ & jafemrSSSSSSSSSSSSSSnode & $\begin{array}{r}1287989 \\
1288049 \\
639+30\end{array}$ & $\begin{array}{l}\mathrm{F} \\
27 \mathrm{~N} \\
13 \mathrm{~W} \\
12\end{array}$ & $\begin{array}{ll}\text { A B } & \text { E } \\
\text { F } & \end{array}$ \\
\hline $\begin{array}{c}244.84 \\
97\end{array}$ & $\begin{array}{l}\text { (Jackson Slough) } \\
\text { TAPS A/G; CMP }\end{array}$ & $\begin{array}{l}\mathrm{CN} ; \mathrm{GR} ; \\
\mathrm{KS} ; \mathrm{RW}\end{array}$ & jafemrCCCCCCSSSSCCCCnode & $\begin{array}{r}1292730 \\
1292799 \\
593+00\end{array}$ & $\begin{array}{l}\mathrm{F} \\
27 \mathrm{~N} \\
13 \mathrm{~W} \\
11\end{array}$ & $\begin{array}{l}\text { A B D E } \\
\text { F }\end{array}$ \\
\hline $\begin{array}{c}245.26 \\
97\end{array}$ & $\begin{array}{l}\text { (Jackson Slough) } \\
\text { TAPS A/G; CMP }\end{array}$ & $\begin{array}{l}\mathrm{CN} ; \mathrm{GR} ; \\
\mathrm{KS} ; \mathrm{RW}\end{array}$ & jafemrCCCCCCSSSSCCCCnode & $\begin{array}{r}1294964 \\
1295024 \\
570+78\end{array}$ & $\begin{array}{l}\mathrm{F} \\
27 \mathrm{~N} \\
13 \mathrm{~W} \\
14 \\
\end{array}$ & $\begin{array}{l}\text { A B DE } \\
F\end{array}$ \\
\hline
\end{tabular}


USDOI BLM PMO OPEN FILE REPORT - TAPS FISH STREAMS

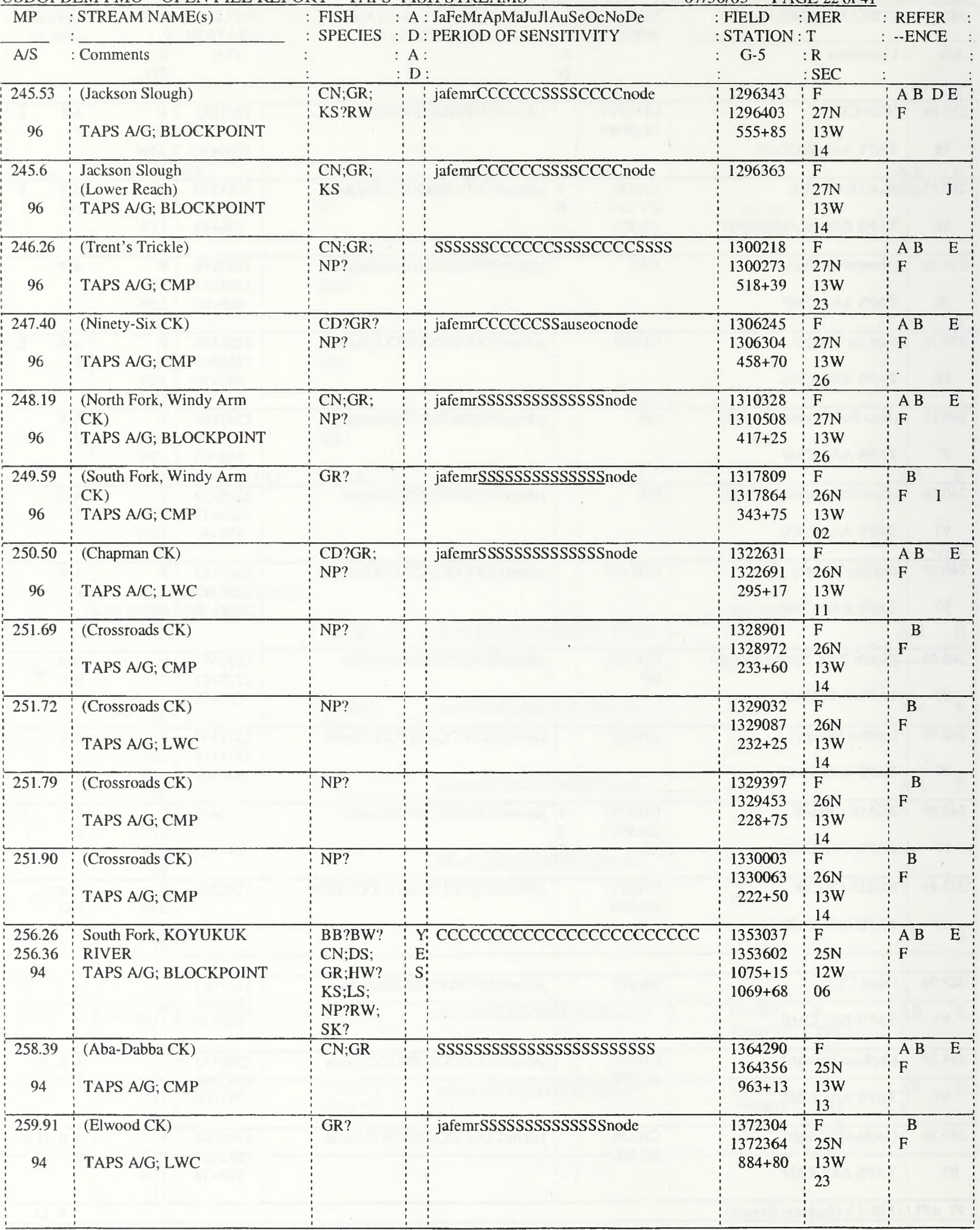


USDOI BLM PMO OPEN FILE REPORT - TAPS FISH STREAMS

07/30/03 PAGE 23 of 41

\begin{tabular}{|c|c|c|c|c|c|c|}
\hline MP & STREAM NAME(s) & $\begin{array}{l}\text { FISH } \\
\text { SPECIES }\end{array}$ & $\begin{array}{l}\text { A : JaFeMrApMaJuJIAuSeOcNoDe } \\
\text { D : PERIOD OF SENSITIVITY }\end{array}$ & $\begin{array}{l}\text { FIELD } \\
\text { STATION }\end{array}$ & $\begin{array}{l}\text { MER } \\
T\end{array}$ & $\begin{array}{l}\text { REFER } \\
\text {--ENCE }\end{array}$ \\
\hline AVS & Comments & & & G-5 & $\begin{array}{l}: \mathrm{R} \\
: \text { SEC }\end{array}$ & \\
\hline $\begin{array}{c}268.12 \\
92\end{array}$ & $\begin{array}{l}\text { JIM RIVER \#3 } \\
\text { TAPS B/G; BLOCKPOINT }\end{array}$ & $\begin{array}{l}\text { BB;CN;DS } \\
\text { GR;HW; } \\
\text { KS;LS;NP; } \\
\text { RW }\end{array}$ & $\begin{array}{l}\mathrm{E}_{1} \text { CCCCCCCCCCCCCCCCCCCCCCCC } \\
\mathrm{S}_{1} \\
\vdots\end{array}$ & $\begin{array}{r}1415550 \\
1415800 \\
453+50\end{array}$ & $\begin{array}{l}\mathrm{F} \\
24 \mathrm{~N} \\
14 \mathrm{~W} \\
23 \& 26\end{array}$ & $\begin{array}{l}\text { A B } \quad \text { E } \\
\text { F G }\end{array}$ \\
\hline $\begin{array}{c}268.44 \\
92\end{array}$ & $\begin{array}{l}\text { (Beaver Spring) } \\
\text { TAPS A/G; CMP }\end{array}$ & $\begin{array}{l}\text { CN?GR? } \\
\text { KS?RW? }\end{array}$ & CCCCCCCCCCCCSSSSCCCCCCCC & $\begin{array}{r}1417312 \\
1417426 \\
435+84\end{array}$ & $\begin{array}{l}\mathrm{F} \\
24 \mathrm{~N} \\
14 \mathrm{~W} \\
26\end{array}$ & \\
\hline $\begin{array}{c}269.00 \\
92\end{array}$ & $\begin{array}{l}\text { (Dee CK) } \\
\text { TAPS A/G; CMP }\end{array}$ & $\begin{array}{l}\mathrm{CN} ; \mathrm{DV} ? \\
\mathrm{GR} ; \mathrm{RW}\end{array}$ & SSSSSSSSSSSSSSSSSSSSSSSS & $\begin{array}{r}1420293 \\
1420353 \\
406+86\end{array}$ & $\begin{array}{l}\mathrm{F} \\
24 \mathrm{~N} \\
14 \mathrm{~W} \\
26\end{array}$ & $\begin{array}{l}\mathrm{A} \text { B } \\
\text { F }\end{array}$ \\
\hline $\begin{array}{c}270.45 \\
92\end{array}$ & $\begin{array}{l}\text { DOUGLAS CREEK } \\
\text { TAPS A/G; LWC }\end{array}$ & $\begin{array}{l}\text { CN;GR; } \\
\text { RW? }\end{array}$ & jafemrSSSSSSSSSSSSSSnode & $\begin{array}{r}1427745 \\
1428168 \\
333+75\end{array}$ & $\begin{array}{l}F \\
24 N \\
14 W \\
34\end{array}$ & $\begin{array}{l}\mathrm{A} B \\
\mathrm{~F}\end{array}$ \\
\hline $\begin{array}{c}271.31 \\
92\end{array}$ & $\begin{array}{l}\text { (Gas Bubble Slough) } \\
\text { TAPS A/G; Parallel Only } \\
\text { TAPS DOES NOT CROSS } \\
\end{array}$ & GR & 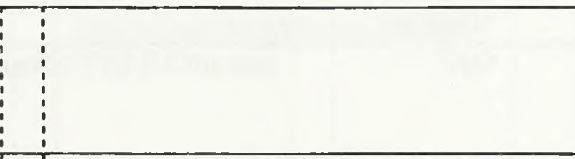 & $289+00$ & $\begin{array}{l}\mathrm{F} \\
23 \mathrm{~N} \\
14 \mathrm{~W} \\
03\end{array}$ & A B ${ }_{1}$ \\
\hline $\begin{array}{c}271,62 \\
92\end{array}$ & $\begin{array}{l}\text { Side Channel JIM RIVER \#2 } \\
\text { TAPS B/G; BLOCKPOINT }\end{array}$ & $\begin{array}{l}\text { BB;CN;DS } \\
\text { GR;HW; } \\
\text { KS:LS;NP; } \\
\text { RW }\end{array}$ & $\begin{array}{l:} \\
\end{array}$ & $\begin{array}{r}1434078 \\
1434178 \\
272+51\end{array}$ & $\begin{array}{l}\mathrm{F} \\
23 \mathrm{~N} \\
14 \mathrm{~W} \\
03\end{array}$ & $\begin{array}{l}\text { A B } \quad \text { E } \\
\text { F }\end{array}$ \\
\hline $\begin{array}{c}271.92 \\
92\end{array}$ & $\begin{array}{l}\text { Side Channel JIM RIVER } \\
\# 1 \\
\text { TAPS B/G; BLOCKPOINT }\end{array}$ & $\begin{array}{l}\text { BB;CN;DS; } \\
\text { GR;HW; } \\
\text { KS;LS;NP; } \\
\text { RW }\end{array}$ & 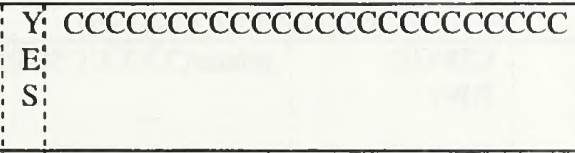 & $\begin{array}{r}1435700 \\
1435800 \\
257+00\end{array}$ & $\begin{array}{l}\mathrm{F} \\
24 \mathrm{~N} \\
14 \mathrm{~W} \\
09\end{array}$ & $\begin{array}{l}\text { A B } \\
\text { F }\end{array}$ \\
\hline $\begin{array}{c}272.21 \\
91\end{array}$ & $\begin{array}{l}\text { (Little Piddler) } \\
\text { TAPS A/G; CMP }\end{array}$ & $\overline{G R}$ & jafemrSSSSSSSSSSSSSSnode & $\begin{array}{r}1437254 \\
1437307 \\
240+85\end{array}$ & $\begin{array}{l}\mathrm{F} \\
23 \mathrm{~N} \\
14 \mathrm{~W} \\
09\end{array}$ & $\begin{array}{l}\text { A B } \\
\text { F }\end{array}$ \\
\hline
\end{tabular}


USDOI BLM PMO OPEN FILE REPORT - TAPS FISH STREAMS

07/30/03 PAGE 24 of 41

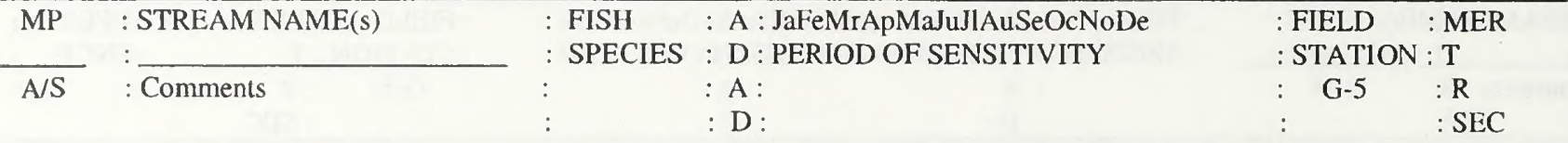

\begin{tabular}{|c|c|c|c|c|c|c|}
\hline $\begin{array}{c}277.14 \\
91\end{array}$ & $\begin{array}{l}\text { PROSPECT CREEK } \\
\text { TAPS A/G; BLOCKPOINT }\end{array}$ & $\begin{array}{l}\text { CN;GR; } \\
\text { KS;LS; } \\
\text { NP;RW }\end{array}$ & $\begin{array}{l:l}\mathrm{CCCCCCCCCCCCCCCCCCCCCCCC} \\
\mathrm{Y}\end{array}$ & $\begin{array}{l}1463150 \\
1463408 \\
1590+00\end{array}$ & $\begin{array}{l}\mathrm{F} \\
23 \mathrm{~N} \\
14 \mathrm{~W} \\
31 \\
\end{array}$ & $\begin{array}{ll}\text { A B } \\
\text { F G }\end{array}$ \\
\hline $\begin{array}{c}281.88 \\
90\end{array}$ & $\begin{array}{l}\text { (Little Nasty CK) } \\
\text { TAPS A/G; BLOCKPOINT }\end{array}$ & $\begin{array}{l}\mathrm{CN} ; \mathrm{GR} ; \\
\mathrm{RW}\end{array}$ & jafemrSSSSSSSSSSSSSSnode & $\begin{array}{l}1488219 \\
1488452 \\
1339+00\end{array}$ & $\begin{array}{l}\mathrm{F} \\
22 \mathrm{~N} \\
15 \mathrm{~W} \\
19\end{array}$ & $\begin{array}{l}\mathrm{AB} \\
\mathrm{F}\end{array}$ \\
\hline $\begin{array}{c}282.14 \\
90\end{array}$ & $\begin{array}{l}\text { (South Fork, Little Nasty CK) } \\
\text { TAPS A/G; CMP }\end{array}$ & CD?GR & jafemrSSSSSSSSSSSSSSnode & $\begin{array}{l}1489653 \\
1489705 \\
1327+40\end{array}$ & $\begin{array}{l}\mathrm{F} \\
22 \mathrm{~N} \\
15 \mathrm{~W} \\
19\end{array}$ & $\begin{array}{ll}\text { A B } & \text { E } \\
F & \end{array}$ \\
\hline $\begin{array}{c}284.40 \\
89\end{array}$ & $\begin{array}{l}\text { North Fork BONANZA } \\
\text { CREEK } \\
\text { TAPS B/G; BLOCKPOINT }\end{array}$ & $\begin{array}{l}\mathrm{BB} ; \mathrm{CN} ; \\
\mathrm{GR} ; \mathrm{HW} ? \\
\mathrm{LS} ; \text {;LW; } \\
\mathrm{NP} ; \mathrm{RW}\end{array}$ & jafemrCCCCCCSSSSCCCCnode & $\begin{array}{l}1501503 \\
1501783 \\
1208+32\end{array}$ & $\begin{array}{l}\mathrm{F} \\
22 \mathrm{~N} \\
14 \mathrm{~W} \\
32 \\
\end{array}$ & $\begin{array}{ll}\text { A B } & \text { E } \\
\text { F G } & \end{array}$ \\
\hline $\begin{array}{c}285.53 \\
89\end{array}$ & $\begin{array}{l}\text { (Oxbow Lake System) } \\
\text { TAPS B/G; CMP }\end{array}$ & GR? & jafemrCCCCCCSSauseocnode & $\begin{array}{l}1507600 \\
1148+00\end{array}$ & $\begin{array}{l}\mathrm{F} \\
21 \mathrm{~N} \\
14 \mathrm{~W} \\
07 \\
\end{array}$ & $\begin{array}{l}\text { A B } \\
F\end{array}$ \\
\hline $\begin{array}{c}286.01 \\
89\end{array}$ & $\begin{array}{l}\text { South Fork BONANZA } \\
\text { CREEK } \\
\text { TAPS B/G; BLOCKPOINT }\end{array}$ & $\begin{array}{l}\mathrm{BB} ; \mathrm{CN} ; \\
\mathrm{GR} ; \mathrm{HW} ? \\
\mathrm{LS} ; \mathrm{NP} ; \mathrm{RW}\end{array}$ & SSSSSSCCCCCCSSSCCCCCCCSS & $\begin{array}{l}1510050 \\
1510200 \\
1123+60\end{array}$ & $\begin{array}{l}\mathrm{F} \\
21 \mathrm{~N} \\
14 \mathrm{~W} \\
07 \\
\end{array}$ & $\begin{array}{l}\mathrm{AB} \\
\mathrm{F} \mathrm{G}\end{array}$ \\
\hline $\begin{array}{c}289.63 \\
88\end{array}$ & $\begin{array}{l}\text { (Pung's Crossing CK) } \\
\text { TAPS A/G; BRIDGE }\end{array}$ & $\begin{array}{l}\text { CD?GR; } \\
\text { RW? }\end{array}$ & jafemrCCCCCCSSSSCCCCnode & $\begin{array}{r}1529195 \\
1529325 \\
932+60 \\
932+10 \\
\end{array}$ & $\begin{array}{l}\mathrm{F} \\
21 \mathrm{~N} \\
14 \mathrm{~W} \\
31 \\
\end{array}$ & $\begin{array}{l}\text { A B } \\
\text { F }\end{array}$ \\
\hline $\begin{array}{c}293.22 \\
88\end{array}$ & $\begin{array}{l}\text { (Alder Mountain CK) } \\
\text { TAPS B/G; LWC }\end{array}$ & $\begin{array}{l}\text { CN;GR? } \\
\text { RW? }\end{array}$ & jafemrSSSSSSSSSSSSSSnode & $\begin{array}{r}1548200 \\
742+50\end{array}$ & $\begin{array}{l}\mathrm{F} \\
20 \mathrm{~N} \\
15 \mathrm{~W} \\
10 \\
\end{array}$ & $\begin{array}{l}\text { A B } \\
\text { F }\end{array}$ \\
\hline 294.91 & $\begin{array}{l}\text { FISH CREEK } \\
\text { TAPS A/G;BRIDGE }\end{array}$ & $\begin{array}{l}\text { BW?CN; } \\
\text { DS?GR; } \\
\text { LS;NP? } \\
\text { RW;SK }\end{array}$ & jafemrCCCCCCSSSSCCCCnode & $\begin{array}{r}1557094 \\
1557163 \\
653+50\end{array}$ & $\begin{array}{l}\mathrm{F} \\
20 \mathrm{~N} \\
15 \mathrm{~W} \\
22 \\
\end{array}$ & $\begin{array}{l}\text { A B } \\
\text { F }\end{array}$ \\
\hline $\begin{array}{c}296.34 \\
87\end{array}$ & $\begin{array}{l}\text { Middle Fork, FISH CREEK } \\
\text { TAPS A/G; CMP }\end{array}$ & $\begin{array}{l}\text { CN;GR; } \\
\text { RW }\end{array}$ & jafemrSSSSSSSSSSSSSSnode & $\begin{array}{r}1564650 \\
578+00\end{array}$ & $\begin{array}{l}\mathrm{F} \\
20 \mathrm{~N} \\
15 \mathrm{~W} \\
26 \\
\end{array}$ & $\begin{array}{l}\mathrm{AB} \\
\mathrm{F}\end{array}$ \\
\hline $\begin{array}{c}297.42 \\
87\end{array}$ & $\begin{array}{l}\text { South Fork FISH CREEK } \\
\text { TAPS A/G; BRIDGE }\end{array}$ & $\begin{array}{l}\text { CN;GR; } \\
\text { RW? }\end{array}$ & SSSSSSCCCCCCSSSSCCCCCCSS & $\begin{array}{r}1570361 \\
1570423 \\
520+60\end{array}$ & $\begin{array}{l}\mathrm{F} \\
20 \mathrm{~N} \\
15 \mathrm{~W} \\
35 \\
\end{array}$ & $\begin{array}{l}\text { A B } \\
F\end{array}$ \\
\hline $\begin{array}{c}302.92 \\
86\end{array}$ & $\begin{array}{l}\text { KANUTI RIVER } \\
\text { TAPS B/G; BLOCKPOINT }\end{array}$ & $\begin{array}{l}\text { BB;BC? } \\
\text { BW?CN; } \\
\text { CS?DS? } \\
\text { GR;HW? } \\
\text { IN?LS? } \\
\text { NP;RW }\end{array}$ & jafemrCCCCCCSSSSCCCCnode & $\begin{array}{r}1599300 \\
1599500 \\
231+00\end{array}$ & $\begin{array}{l}\mathrm{F} \\
19 \mathrm{~N} \\
14 \mathrm{~W} \\
30\end{array}$ & $\begin{array}{l}\text { A B } \\
F\end{array}$ \\
\hline
\end{tabular}

\begin{tabular}{|c|c|c|c|c|c|c|}
\hline $\begin{array}{c}306.22 \\
86\end{array}$ & $\begin{array}{l}\text { (Caribou Mountain CK) } \\
\text { TAPS B.G; BLOCKPOINT }\end{array}$ & GR & jafemrSSSSSSSSSSSSSSnode & $\begin{array}{r}1616819 \\
1616887 \\
56+03\end{array}$ & $\begin{array}{l}\mathrm{F} \\
18 \mathrm{~N} \\
14 \mathrm{~W} \\
09\end{array}$ & $\begin{array}{ll}\text { A B } & \text { E } \\
\text { F } & \end{array}$ \\
\hline $\begin{array}{c}308.56 \\
85\end{array}$ & $\begin{array}{l}\text { (Olson's Lake CK) } \\
\text { TAPS A/G; BLOCKPOINT }\end{array}$ & GR & jafemrSSSSSSSSSSSSSSnode & $\begin{array}{l}1629150 \\
1629200 \\
1149+39\end{array}$ & $\begin{array}{l}\mathrm{F} \\
18 \mathrm{~N} \\
14 \mathrm{~W} \\
14 \\
\end{array}$ & $\begin{array}{ll}\text { A B } & \text { E } \\
\text { F } & \end{array}$ \\
\hline
\end{tabular}


USDOI BLM PMO OPEN FILE REPORT - TAPS FISH STREAMS

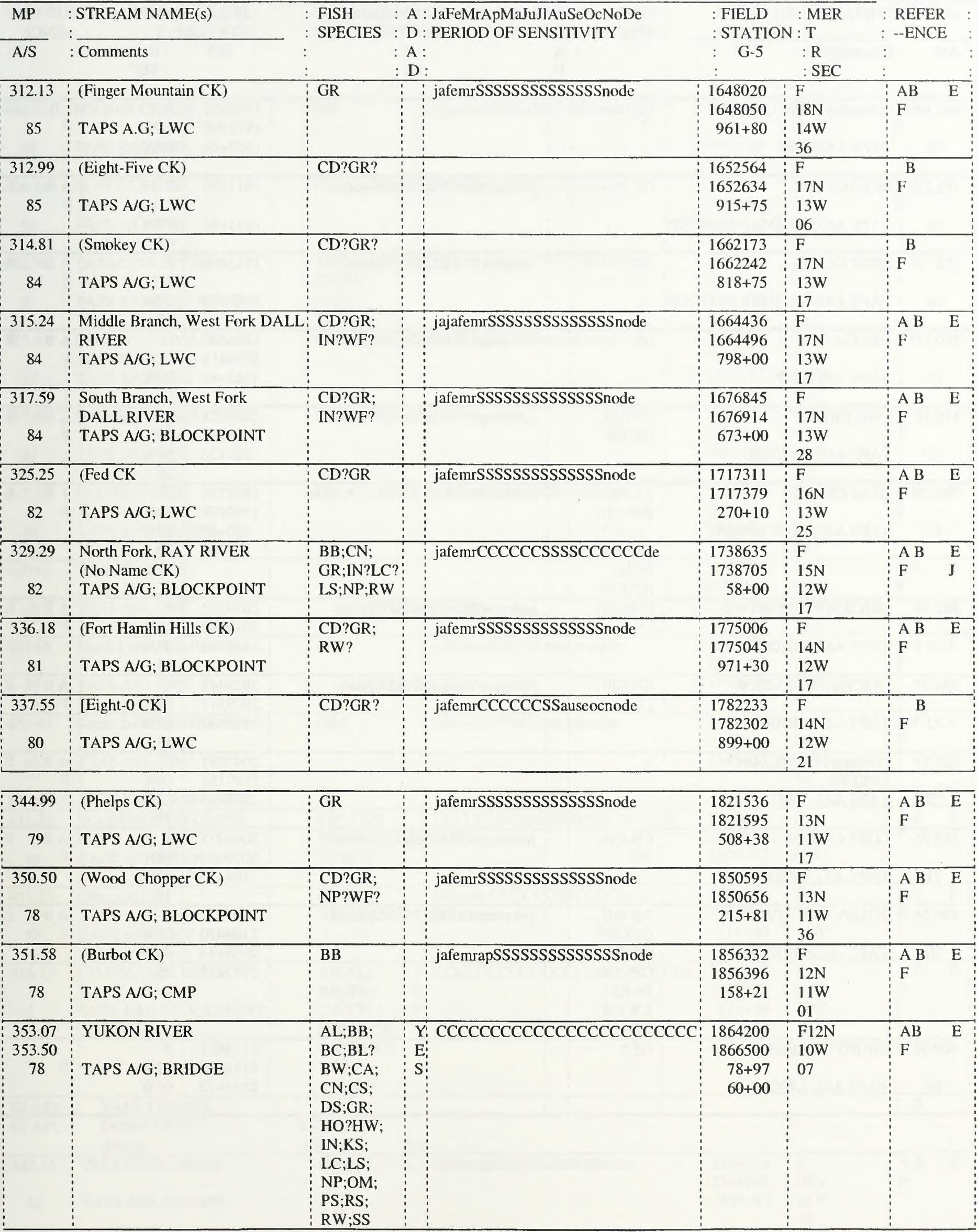


USDOI BLM PMO OPEN FILE REPORT - TAPS FISH STREAMS

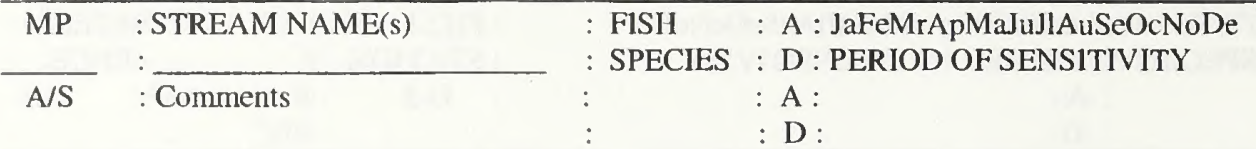

\begin{tabular}{|c|c|c|c|c|c|c|}
\hline $\begin{array}{c}362.14 \\
76\end{array}$ & $\begin{array}{l}\text { (Tributary ISOM CREEK) } \\
\text { TAPS A/G; CMP }\end{array}$ & GR? & & $\begin{array}{l}1912052 \\
1912106 \\
1682+08\end{array}$ & $\begin{array}{l}\mathrm{F} \\
11 \mathrm{~N} \\
10 \mathrm{~W} \\
12\end{array}$ & $F^{B}$ \\
\hline $\begin{array}{c}362.72 \\
76\end{array}$ & $\begin{array}{l}\text { ISOM CREEK } \\
\text { TAPS A/G; SUMMER BLOCKPC }\end{array}$ & GR & jafemrapSSSSSSSSSSSSnode & $\begin{array}{l}1915150 \\
1651+65\end{array}$ & $\begin{array}{l}\mathrm{F} \\
11 \mathrm{~N} \\
09 \mathrm{~W} \\
07\end{array}$ & $\begin{array}{ll}\mathrm{AB} & \mathrm{E} \\
\mathrm{F} & \end{array}$ \\
\hline $\begin{array}{c}362.76 \\
76\end{array}$ & $\begin{array}{l}\text { ISOM CREEK } \\
\text { TAPS A/G; SUMMER BLOCKPC }\end{array}$ & $\overline{\mathrm{GR}}$ & jafemrapSSSSSSSSSSSSnode & $\begin{array}{l}1915400 \\
1642+39\end{array}$ & $\begin{array}{l}\mathrm{F} \\
11 \mathrm{~N} \\
09 \mathrm{~W} \\
07\end{array}$ & $\begin{array}{ll}\text { A B } & \text { E } \\
\text { F } & \end{array}$ \\
\hline $\begin{array}{c}370.53 \\
75\end{array}$ & $\begin{array}{l}\text { (Hot Cat CK) } \\
\text { TAPS A/G; CMP }\end{array}$ & $\overline{G R}$ & jafemrapSSSSSSSSSSSSnode & $\begin{array}{l}1956346 \\
1956416 \\
1242+46\end{array}$ & $\begin{array}{l}\mathrm{F} \\
11 \mathrm{~N} \\
08 \mathrm{~W} \\
31\end{array}$ & $\begin{array}{ll}\text { A B } & \text { E } \\
\text { F } & \end{array}$ \\
\hline $\begin{array}{c}378.39 \\
73\end{array}$ & $\begin{array}{l}\text { FISH CREEK } \\
\text { TAPS A/G; BRIDGE }\end{array}$ & $\begin{array}{l}\text { CN?GR; } \\
\text { NP;WF }\end{array}$ & jafemrapSSSSSSSSSSSSnode & $\begin{array}{r}1997824 \\
1997932 \\
829+56\end{array}$ & $\begin{array}{l}\mathrm{F} \\
10 \mathrm{~N} \\
07 \mathrm{~W} \\
20\end{array}$ & $\begin{array}{ll}\text { A B } & \text { E } \\
F\end{array}$ \\
\hline $\begin{array}{c}378.57 \\
73\end{array}$ & $\begin{array}{l}\text { HESS CREEK } \\
\text { TAPS A/G; BLOCKPOINT }\end{array}$ & $\begin{array}{l}\text { AL?BC; } \\
\text { BW;CN; } \\
\text { CS;DS? } \\
\text { GR;HW; } \\
\text { IN;LS; } \\
\text { NP;RW }\end{array}$ & jafemrCCCCCCCCCCCCCCnode & $\begin{array}{r}1998734 \\
1998928 \\
820+49 \\
819+31\end{array}$ & $\begin{array}{l}\mathrm{F} \\
10 \mathrm{~N} \\
07 \mathrm{~W} \\
20\end{array}$ & $\begin{array}{l}\text { A B. } \\
\text { F }\end{array}$ \\
\hline $\begin{array}{c}382.50 \\
73\end{array}$ & $\begin{array}{l}\text { ERICKSON CREEK \# } 2 \\
\text { TAPS A/G; BRIDGE }\end{array}$ & $\begin{array}{l}\text { CN?GR; } \\
\text { LS }\end{array}$ & jafemrapSSSSSSSSSSSSnode & $\begin{array}{r}2019562 \\
2019642 \\
611+95\end{array}$ & $\begin{array}{l}\mathrm{F} \\
09 \mathrm{~N} \\
07 \mathrm{~W} \\
03\end{array}$ & $\begin{array}{ll}\text { A B } & \text { E } \\
F & \end{array}$ \\
\hline $\begin{array}{c}384.37 \\
72\end{array}$ & $\begin{array}{l}\text { ERICKSON CREEK \# } 1 \\
\text { TAPS A/G; BRIDGE }\end{array}$ & $\begin{array}{l}\text { CN?GR; } \\
\text { LS }\end{array}$ & jafemrapSSSSSSSSSSSSnode & $\begin{array}{r}2029448 \\
2029517 \\
513+62\end{array}$ & $\begin{array}{l}\mathrm{F} \\
09 \mathrm{~N} \\
07 \mathrm{~W} \\
14\end{array}$ & $\begin{array}{ll}\text { A B } & \text { E } \\
\text { F }\end{array}$ \\
\hline $\begin{array}{c}387.71 \\
72\end{array}$ & $\begin{array}{l}\text { (Tributary ERICKSON } \\
\text { CREEK) } \\
\text { TAPS A/G; LWC }\end{array}$ & $?$ & & $\begin{array}{r}2047097 \\
2047158 \\
336+15\end{array}$ & $\begin{array}{l}\mathrm{F} \\
09 \mathrm{~N} \\
06 \mathrm{~W} \\
30 \\
\end{array}$ & $\begin{array}{ll}\mathrm{AB} & \mathrm{E} \\
\mathrm{F} & \end{array}$ \\
\hline $\begin{array}{c}392.05 \\
71\end{array}$ & $\begin{array}{l}\text { LOST CREEK } \\
\text { TAPS A/G; BRIDGE }\end{array}$ & $\begin{array}{l}\text { CN;GR; } \\
\text { WF }\end{array}$ & jafemrapSSSSSSSSSSSSnode & $\begin{array}{r}2069975 \\
2070055 \\
104+33\end{array}$ & $\begin{array}{l}\mathrm{F} \\
08 \mathrm{~N} \\
06 \mathrm{~W} \\
16 \\
\end{array}$ & $\begin{array}{l}\mathrm{A} \mathrm{B} \\
\mathrm{F}\end{array}$ \\
\hline $\begin{array}{c}398.56 \\
70\end{array}$ & $\begin{array}{l}\text { TOLOVANA RIVER } \\
\text { TAPS A/G; BRIDGE }\end{array}$ & $\begin{array}{l}\text { AB?BB; } \\
\text { CI?CN? } \\
\text { CS?DS; } \\
\text { GR;HW; } \\
\text { IN;KS; } \\
\text { LW?NP; } \\
\text { SS? }\end{array}$ & jafemrapSSSSSSSSSSSSSSSS & $\begin{array}{l}2104372 \\
2104470 \\
2958+13 \\
2957+13\end{array}$ & $\begin{array}{l}\mathrm{F} \\
07 \mathrm{~N} \\
05 \mathrm{~W} \\
05\end{array}$ & $\begin{array}{l}\text { A B } \\
F\end{array}$ \\
\hline $\begin{array}{c}400.50 \\
69\end{array}$ & $\begin{array}{l}\text { SHORTY CREEK } \\
\text { TAPS A/G; LWC }\end{array}$ & GR? & & $\begin{array}{l}2114631 \\
2114699 \\
2855+73\end{array}$ & $\begin{array}{l}\mathrm{F} \\
07 \mathrm{~N} \\
05 \mathrm{~W} \\
09\end{array}$ & $F^{B}$ \\
\hline
\end{tabular}

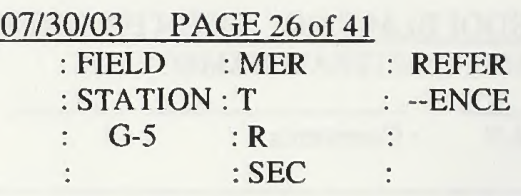

\begin{tabular}{|c|c|}
\hline FIELD & : MER \\
\hline : STATION & $: \mathrm{T}$ \\
\hline : G-5 & $: \mathbf{R}$ \\
\hline & :SEC \\
\hline
\end{tabular}


USDOI BLM PMO OPEN FILE REPORT - TAPS FISH STREAMS

$\begin{array}{llll}\text { MP } & : \text { STREAM NAME(s) } & : \text { FISH } & : \text { A : JaFeMrApMaJuJIAuSeOcNoDe } \\ & : \text { SPECIES } & : \text { D : PERIOD OF SENSITIVITY } \\ \text { A/S }: \text { Comments } & \vdots & : \text { A : } \\ & & : & \text { D : }\end{array}$

\begin{tabular}{|c|c|c|c|c|c|c|}
\hline $\begin{array}{c}405.51 \\
68\end{array}$ & $\begin{array}{l}\text { W1LBER CREEK } \\
\text { TAPS B/G; CMP }\end{array}$ & GR & jafemrapSSSSSSSSSSSSnode & $\begin{array}{l}2141100 \\
2609+50\end{array}$ & $\begin{array}{l}\mathrm{F} \\
07 \mathrm{~N} \\
04 \mathrm{~W} \\
30\end{array}$ & $\begin{array}{l}\text { A B } \quad \text { E } \\
\text { F }\end{array}$ \\
\hline $\begin{array}{c}408.36 \\
68\end{array}$ & $\begin{array}{l}\text { SLATE CREEK } \\
\text { TAPS A/G; CMP }\end{array}$ & $\overline{G R}$ & jafemrapSSSSSSSSSSSSnode & $\begin{array}{l}2156110 \\
2156181 \\
2459+42\end{array}$ & $\begin{array}{l}\mathrm{F} \\
06 \mathrm{~N} \\
04 \mathrm{~W} \\
05\end{array}$ & $\begin{array}{l}\text { A B } \\
\text { F }\end{array}$ \\
\hline $\begin{array}{c}412.50 \\
67\end{array}$ & $\begin{array}{l}\text { TATALINA RIVER } \\
\text { TAPS A/G; BLOCKPOINT }\end{array}$ & $\begin{array}{l}\text { BB?CI; } \\
\text { CN?GR; } \\
\text { IN?NP; } \\
\text { SK?WF }\end{array}$ & jafemrapCCCCCCCCSSSSSSde & $\begin{array}{l}2177950 \\
2178000 \\
2242+57 \\
2241+22 \\
\end{array}$ & $\begin{array}{l}\mathrm{F} \\
06 \mathrm{~N} \\
04 \mathrm{~W} \\
26 \\
\end{array}$ & $\begin{array}{l}\text { A B } \\
\text { F }\end{array}$ \\
\hline $\begin{array}{c}413.61 \\
67\end{array}$ & $\begin{array}{l}\text { (Tributary TATALINA } \\
\text { RIVER) } \\
\text { TAPS A/G; LWC }\end{array}$ & GR? & jafemrapSSSSSSauseocnode & $\begin{array}{l}2183830 \\
2183890 \\
2166+87\end{array}$ & $\begin{array}{l}\mathrm{F} \\
06 \mathrm{~N} \\
04 \mathrm{~W} \\
21 \\
\end{array}$ & $\begin{array}{l}\text { A B } \\
\text { F }\end{array}$ \\
\hline $\begin{array}{c}417.00 \\
67\end{array}$ & $\begin{array}{l}\text { (Tributary GLOBE CREEK) } \\
\text { TAPS A/G; LWC }\end{array}$ & GR? & & $\begin{array}{l}2201751 \\
2201811 \\
1988+88\end{array}$ & $\begin{array}{l}\mathrm{F} \\
05 \mathrm{~N} \\
03 \mathrm{~W} \\
05 \\
\end{array}$ & $\mathrm{~F}^{\mathrm{B}}$ \\
\hline $\begin{array}{c}417.41 \\
66\end{array}$ & $\begin{array}{l}\text { GLOBE CREEK } \\
\text { TAPS A/G; BRIDGE }\end{array}$ & GR & jafemrapSSSSSSSSSSSSnode & $\begin{array}{l}2203865 \\
2203934 \\
1966+77\end{array}$ & $\begin{array}{l}\mathrm{F} \\
05 \mathrm{~N} \\
03 \mathrm{~W} \\
08 \\
\end{array}$ & $\begin{array}{l}\text { A B } \\
\text { F }\end{array}$ \\
\hline $\begin{array}{c}420.63 \\
66\end{array}$ & $\begin{array}{l}\text { (Little Globe CK) } \\
\text { TAPS A/G; LWC }\end{array}$ & $?$ & & $\begin{array}{l}2220895 \\
2220953 \\
1759+94\end{array}$ & $\begin{array}{l}\mathrm{F} \\
05 \mathrm{~N} \\
03 \mathrm{~W} \\
22 \\
\end{array}$ & $\begin{array}{ll}\text { A B } & \text { E } \\
\text { F } & \end{array}$ \\
\hline $\begin{array}{c}423.68 \\
65\end{array}$ & $\begin{array}{l}\text { North Fork AGGlE CREEK } \\
\text { TAPS A/G; LWC }\end{array}$ & GR? & jafemrapSSSSSSauseocnode & $\begin{array}{l}2236946 \\
2237126 \\
1635+99\end{array}$ & $\begin{array}{l}\mathrm{F} \\
04 \mathrm{~N} \\
03 \mathrm{~W} \\
03 \\
\end{array}$ & $\begin{array}{ll}\text { A B } & \text { E } \\
\text { F } & \end{array}$ \\
\hline $\begin{array}{c}424.44 \\
65\end{array}$ & $\begin{array}{l}\text { South Fork AGGlE CREEK } \\
\text { TAPS A/G; LWC }\end{array}$ & GR? & jafemrapSSSSSSauseocnode & $\begin{array}{l}2241016 \\
2241077 \\
1595+22\end{array}$ & $\begin{array}{l}\mathrm{F} \\
04 \mathrm{~N} \\
03 \mathrm{~W} \\
10 \\
\end{array}$ & $\begin{array}{ll}\text { A B } & \text { E } \\
\text { F } & \end{array}$ \\
\hline 64 APL-2 & 2 SNOWSHOE CREEK & & & & & B \\
\hline $\begin{array}{c}431.76 \\
64\end{array}$ & $\begin{array}{l}\text { WASHINGTON CREEK } \\
\text { TAPS A/G; BRIDGE }\end{array}$ & $\begin{array}{l}\text { BB? CN? } \\
\text { GR;IN? } \\
\text { NP?RW? }\end{array}$ & CCCCCCCCSSSSSSSSSSCCCCCC & $\begin{array}{l}2279644 \\
2279714 \\
1209+62\end{array}$ & $\begin{array}{l}\mathrm{F} \\
03 \mathrm{~N} \\
02 \mathrm{~W} \\
04 \\
\end{array}$ & $\begin{array}{l}\text { A B } \quad \text { E } \\
F\end{array}$ \\
\hline $\begin{array}{c}437.35 \\
63\end{array}$ & $\begin{array}{l}\text { (Shocker CK) } \\
\text { TAPS A/G; CMP }\end{array}$ & $\begin{array}{l}\mathrm{CN} ; \mathrm{GR} ; \\
\mathrm{RW}\end{array}$ & jafemrapCCCCCCSSCCCCnode & $\begin{array}{r}2309199 \\
2309269 \\
914+00\end{array}$ & $\begin{array}{l}\mathrm{F} \\
03 \mathrm{~N} \\
01 \mathrm{~W} \\
19 \\
\end{array}$ & $\begin{array}{l}\text { A B } \\
\text { F }\end{array}$ \\
\hline $\begin{array}{c}438.12 \\
63\end{array}$ & $\begin{array}{l}\text { CHATAN1KA RIVER } \\
\text { TAPS B/G; BLOCKPOINT }\end{array}$ & $\begin{array}{l}\text { AB;AL; } \\
\text { BB;BW; } \\
\text { CA?CN } \\
\text { CS?DS;GR; } \\
\text { HW;IN;KS; } \\
\text { LS;NP; } \\
\text { RW;SS }\end{array}$ & 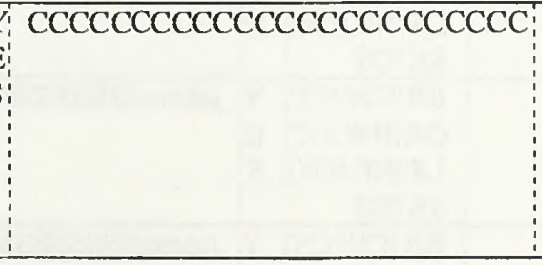 & $\begin{array}{r}2313200 \\
2313300 \\
874+80 \\
873+63\end{array}$ & $\begin{array}{l}\mathrm{F} \\
03 \mathrm{~N} \\
01 \mathrm{~W} \\
29\end{array}$ & $\begin{array}{l}\text { A B } \quad \text { E } \\
\text { F }\end{array}$ \\
\hline 62 APL- & VAULT CREEK & & & & & B \\
\hline 62 APL- & $1 \begin{array}{l}\text { DOME CREEK } \\
\text { Bridge }\end{array}$ & $\begin{array}{l}\mathrm{S} ; \mathrm{DS} ; \mathrm{CN} \\
\mathrm{LS}\end{array}$ & & & & $\mathrm{J}$ \\
\hline $\begin{array}{c}442.18 \\
62\end{array}$ & $\begin{array}{l}\text { TREASURE CREEK } \\
\text { TAPS A/G; BRIDGE }\end{array}$ & $\mathrm{CN} ; \mathrm{GR}$ & jafemrapSSSSSSSSSSSSnode & $\begin{array}{r}2334693 \\
2334762 \\
659+43\end{array}$ & $\begin{array}{l}\mathrm{F} \\
02 \mathrm{~N} \\
01 \mathrm{~W} \\
10 \\
\end{array}$ & $\begin{array}{ll}\text { A B } & \text { E } \\
\text { F } & \end{array}$ \\
\hline
\end{tabular}


USDOI BLM PMO OPEN FILE REPORT - TAPS FISU STREAMS

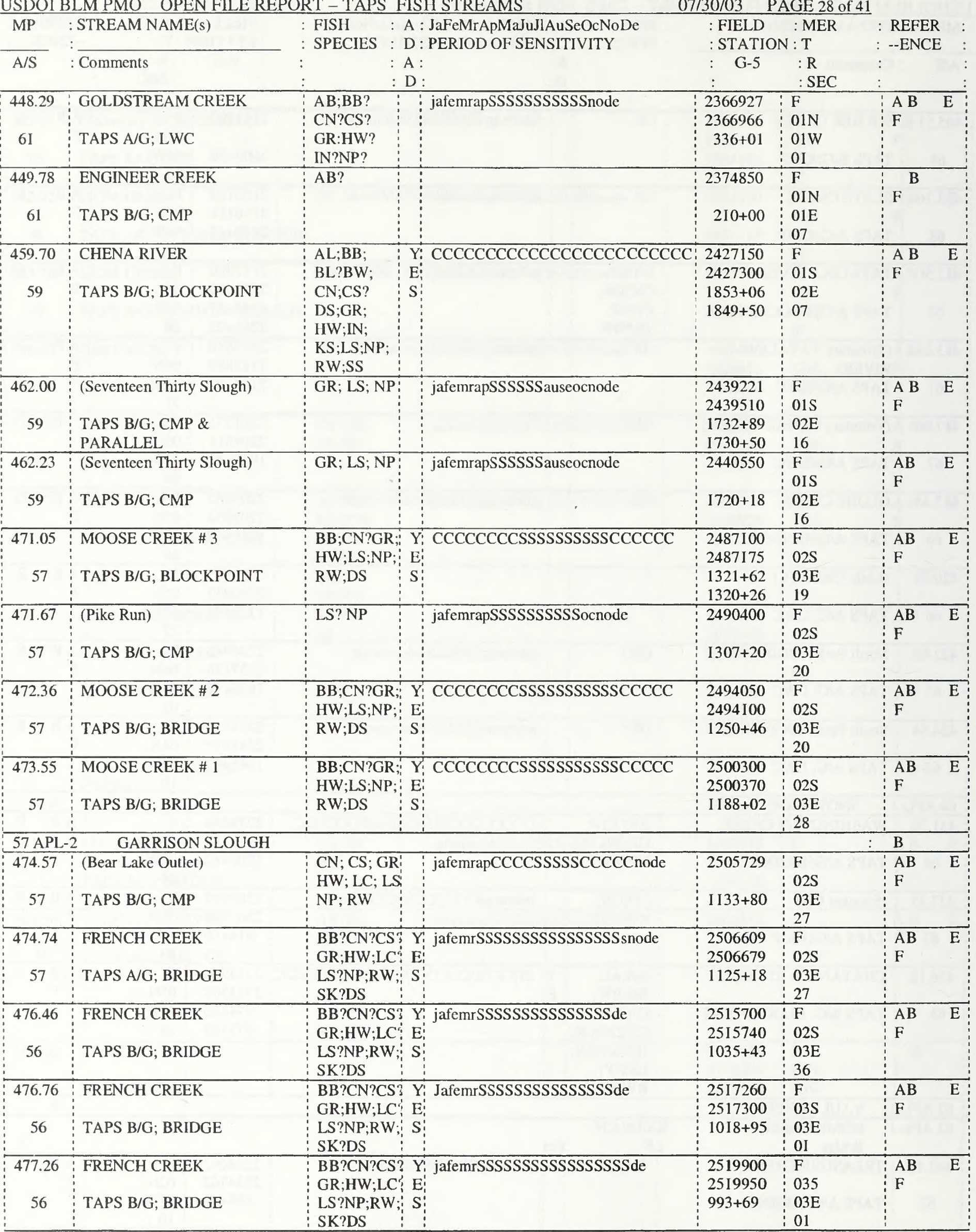


USDOI BLM PMO OPEN FILE REPORT - TAPS FISH STREAMS

07/30/03 PAGE 29 of 41

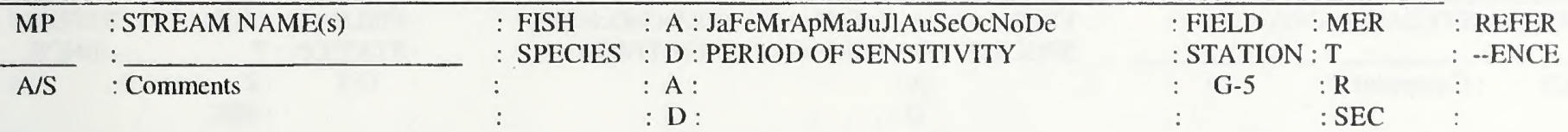

\begin{tabular}{|c|c|c|c|c|c|c|}
\hline $\begin{array}{c}478.20 \\
56\end{array}$ & $\begin{array}{l}\text { FRENCH CREEK } \\
\text { TAPS A/; BRIDGE }\end{array}$ & $\begin{array}{l}\text { BB?CN?CS? } \\
\text { GR;HW;LC; } \\
\text { LS?NP;RW; } \\
\text { SK?DS }\end{array}$ & jafemrSSSSSSSSSSSSSSSSde & $\begin{array}{r}2524868 \\
2524962 \\
942+85\end{array}$ & $\begin{array}{l}\mathrm{F} \\
03 \mathrm{~S} \\
04 \mathrm{E} \\
07\end{array}$ & $\begin{array}{ll}\mathrm{AB} & \mathrm{E} \\
\mathrm{F} & \end{array}$ \\
\hline $56 \mathrm{APL}-$ & FRENCH CREEK & & & & & B \\
\hline $\begin{array}{c}480.75 \\
55\end{array}$ & $\begin{array}{l}\text { (Knokanpeover CK) } \\
\text { TAPS A/G; CMP }\end{array}$ & GR & jafemrapCCCCCCSSCCocnode & $\begin{array}{r}2538317 \\
2538377 \\
809+56\end{array}$ & $\begin{array}{l}\mathrm{F} \\
03 \mathrm{~S} \\
04 \mathrm{E} \\
20\end{array}$ & $\begin{array}{ll}\mathrm{AB} & \mathrm{E} \\
\mathrm{F} & \end{array}$ \\
\hline $\begin{array}{c}483.92 \\
55\end{array}$ & $\begin{array}{l}\text { FRENCH CREEK } \\
\text { TAPS A/G; BRIDGE }\end{array}$ & $\begin{array}{l}\text { BB?CN? } \\
\text { CS?GR; } \\
\text { HW;LC? } \\
\text { LS?NP; } \\
\text { RW;SK? }\end{array}$ & jafemrSSSSSSSSSSSSSSSSde & $\begin{array}{r}2555084 \\
2555154 \\
643+55\end{array}$ & $\begin{array}{l}\mathrm{F} \\
03 \mathrm{~S} \\
04 \mathrm{E} \\
34\end{array}$ & $\begin{array}{ll}\mathrm{AB} & \mathrm{E} \\
\mathrm{F} & \end{array}$ \\
\hline $\begin{array}{c}484.93 \\
55\end{array}$ & $\begin{array}{l}\text { (Million Dollar CK) } \\
\text { TAPS A/G; CMP }\end{array}$ & $\begin{array}{l}\text { CN;GR: } \\
\text { NP;WF }\end{array}$ & jafemrapCCCCCCSSCCocnode & $\begin{array}{r}2560376 \\
2560435 \\
591+55\end{array}$ & $\begin{array}{l}\mathrm{F} \\
04 \mathrm{~S} \\
04 \mathrm{E} \\
02\end{array}$ & $\begin{array}{l}\mathrm{ABC} \quad \mathrm{E} \\
\mathrm{F}\end{array}$ \\
\hline $\begin{array}{c}485.35 \\
55\end{array}$ & $\begin{array}{l}\text { (Million Dollar CK) } \\
\text { TAPS A/G; CMP }\end{array}$ & $\begin{array}{l}\mathrm{CN} ; \mathrm{GR} ; \\
\mathrm{NP} ; \mathrm{WF}\end{array}$ & jafemrapCCCCCCSSCCocnode & $\begin{array}{r}2562587 \\
2562658 \\
568+75\end{array}$ & $\begin{array}{l}\mathrm{F} \\
04 \mathrm{~S} \\
04 \mathrm{E} \\
11\end{array}$ & $\begin{array}{l}\mathrm{ABC} \\
\mathrm{F}\end{array}$ \\
\hline $\begin{array}{c}485.52 \\
55\end{array}$ & $\begin{array}{l}\text { (Million Dollar CK) } \\
\text { TAPS A/G; CMP }\end{array}$ & $\begin{array}{l}\text { CN;GR; } \\
\text { NP;WF }\end{array}$ & jafemrapCCCCCCSSCCocnode & $\begin{array}{r}2563517 \\
2563587 \\
558+65\end{array}$ & $\begin{array}{l}\mathrm{F} \\
04 \mathrm{~S} \\
04 \mathrm{E} \\
11\end{array}$ & $\begin{array}{l}\mathrm{ABC} \quad \mathrm{E} \\
\mathrm{F}\end{array}$ \\
\hline $\begin{array}{c}485.78 \\
55\end{array}$ & $\begin{array}{l}\text { (Million Dollar CK) } \\
\text { TAPS A/G; CMP }\end{array}$ & $\begin{array}{l}\mathrm{CN} ; \mathrm{GR} ; \\
\mathrm{NP} ; \mathrm{WF}\end{array}$ & jafemrapCCCCCCSSCCoenode & $\begin{array}{r}2564873 \\
2564942 \\
545+10\end{array}$ & $\begin{array}{l}\mathrm{F} \\
04 \mathrm{~S} \\
04 \mathrm{E} \\
11\end{array}$ & $\begin{array}{l}A B C E \\
F\end{array}$ \\
\hline $\begin{array}{c}488.24 \\
54\end{array}$ & $\begin{array}{l}\text { (Million Dollar CK) } \\
\text { TAPS A/G; LWC }\end{array}$ & $\begin{array}{l}\text { CN?GR? } \\
\text { NP;WF }\end{array}$ & jafemrapCCCCCCSSCCocnode & $\begin{array}{r}2577851 \\
2577911 \\
417+00\end{array}$ & $\begin{array}{l}\mathrm{F} \\
04 \mathrm{~S} \\
04 \mathrm{E} \\
24\end{array}$ & $F^{B C}$ \\
\hline
\end{tabular}


USDOI BLM PMO OPEN FILE REPORT - TAPS FISH STREAMS

\begin{tabular}{llll}
\hline MP & $:$ STREAM NAME(s) & $:$ FISH & $:$ A : JaFeMrApMaJuJIAuSeOcNoDe \\
& $:$ & $:$ SPECIES & $:$ D : PERIOD OF SENSITIVITY \\
A/S & $:$ Comments & $:$ A : \\
& & $:$ & D :
\end{tabular}

07/30/03 PAGE 30 of 41

$\begin{array}{lll}: \text { FIELD } \quad \text { MER } & : \text { REFER } \\ : \text { STATION }: \text { T } & :--E N C E \\ : \text { G-5 } \quad: \text { R } & : \\ : & : \text { SEC } & :\end{array}$

\begin{tabular}{|c|c|c|c|c|c|c|}
\hline $\begin{array}{c}489.68 \\
54\end{array}$ & $\begin{array}{l}\text { (Tributary LITTLE SALCHA } \\
\text { RIVER) } \\
\text { TAPS A/G; CMP }\end{array}$ & GR? & jafemrSSCCCCSSSSSSocnode & $\begin{array}{r}2585488 \\
2585548 \\
345+50\end{array}$ & $\begin{array}{l}\mathrm{F} \\
04 \mathrm{~S} \\
05 \mathrm{E} \\
30 \\
\end{array}$ & $\mathrm{~F}^{\mathrm{B}}$ \\
\hline $\begin{array}{c}490.84 \\
54\end{array}$ & $\begin{array}{l}\text { LITTLE SALCHA RIVER } \\
\text { TAPS A/G; BRIDGE }\end{array}$ & $\begin{array}{l}\text { BB?CN; } \\
\text { DS;GR; } \\
\text { KS;LS? } \\
\text { NP?WF }\end{array}$ & 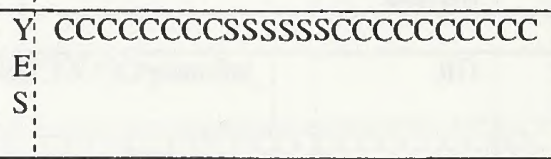 & $\begin{array}{r}2591589 \\
2591659 \\
281+71\end{array}$ & $\begin{array}{l}\mathrm{F} \\
04 \mathrm{~S} \\
05 \mathrm{E} \\
32\end{array}$ & $\begin{array}{ll}\mathrm{AB} & \mathrm{E} \\
\mathrm{F} & \end{array}$ \\
\hline $\begin{array}{r}491.94 \\
53 \mathrm{~A}\end{array}$ & $\begin{array}{l}\text { (Kanpeover or Two-Nineteen } \\
\text { CK) } \\
\text { TAPS A/G; CMP }\end{array}$ & GR & jafemrapCCCCSSauseocnode & $\begin{array}{r}2597387 \\
2597452 \\
219+00\end{array}$ & $\begin{array}{l}\mathrm{F} \\
05 \mathrm{~S} \\
05 \mathrm{E} \\
04 \\
\end{array}$ & $\mathrm{AB} \quad \mathrm{E}$ \\
\hline $\begin{array}{r}495.83 \\
53 \mathrm{~A}\end{array}$ & $\begin{array}{l}\text { SALCHA RIVER } \\
\text { TAPS B/G; BLOCKPOINT }\end{array}$ & $\begin{array}{l}\text { AL;BB; } \\
\text { CN;DS; } \\
\text { GR;KS; } \\
\text { LS;NP; } \\
\text { RW;SB? } \\
\text { SS }\end{array}$ & $\begin{array}{l}\text { Y: CCCCCCCCCCCCCCCCCCCCCCC } \\
\mathrm{E}: \mathrm{C} \\
\mathrm{S}:\end{array}$ & $\begin{array}{r}2617900 \\
2618100 \\
20+65 \\
19+00\end{array}$ & $\begin{array}{l}\mathrm{F} \\
05 \mathrm{~S} \\
05 \mathrm{E} \\
13\end{array}$ & $\begin{array}{ll}\mathrm{AB} & \mathrm{E} \\
\mathrm{F} & \end{array}$ \\
\hline $\begin{array}{r}496.06 \\
53 \mathrm{~A}\end{array}$ & $\begin{array}{l}\text { (Fifty-Three-A CK) } \\
\text { TAPS B/G; LWC HAS BEEN } \\
\text { FILLED }\end{array}$ & $\mathrm{CN} ; \mathrm{DS}$ & $\begin{array}{c}\text { CCCCCCCCSSSSSSCCCCCCCCCC } \\
0\end{array}$ & $\begin{array}{r}2619200 \\
7+50\end{array}$ & $\begin{array}{l}\mathrm{F} \\
05 \mathrm{~S} \\
05 \mathrm{E} \\
13 \\
\end{array}$ & $\mathrm{~F}^{\mathrm{B}}$ \\
\hline $\begin{array}{r}496.16 \\
53 \mathrm{~A}\end{array}$ & $\begin{array}{l}\text { (Taps Slough) } \\
\text { TAPS B/G; CMP }\end{array}$ & $\begin{array}{l}\text { BB?CD? } \\
\text { DS?WF? } \\
\text { KS }\end{array}$ & $\begin{array}{c:c}\text { Y jafemrapSSSSSSSSSSSSnode } \\
E^{\prime}\end{array}$ & $\begin{array}{r}2619700 \\
2+00\end{array}$ & $\begin{array}{l}\mathrm{F} \\
05 \mathrm{~S} \\
05 \mathrm{E} \\
13\end{array}$ & $\begin{array}{ll}\mathrm{AB} & \mathrm{E} \\
\mathrm{F} & \mathrm{J}\end{array}$ \\
\hline $\begin{array}{c}500.02 \\
53\end{array}$ & $\begin{array}{l}\text { REDMOND CREEK } \\
\text { TAPS A/G; BRIDGE }\end{array}$ & $\begin{array}{l}\text { BB;CN; } \\
\text { CS?DS? } \\
\text { GR;KS; } \\
\text { RW;SK? }\end{array}$ & Y jafemrapCCCCSSSSCCCCnode & $\begin{array}{l}2640084 \\
2640154 \\
1085533\end{array}$ & $\begin{array}{l}\mathrm{F} \\
05 \mathrm{~S} \\
06 \mathrm{E} \\
22\end{array}$ & $\begin{array}{ll}\mathrm{AB} & \mathrm{E} \\
\mathrm{F}\end{array}$ \\
\hline $\begin{array}{c}507.03 \\
51\end{array}$ & $\begin{array}{l}\text { GOLD RUN } \\
\text { TAPS A/G; CMP }\end{array}$ & GR & jafemrapSSSSSSSSSSScnode & $\begin{array}{l}2677082 \\
2677148 \\
1048725\end{array}$ & $\begin{array}{l}\mathrm{F} \\
06 \mathrm{~S} \\
07 \mathrm{E} \\
03 \\
\end{array}$ & $\begin{array}{ll}\mathrm{AB} & \mathrm{E} \\
\mathrm{F} & \end{array}$ \\
\hline 508.79 & $\begin{array}{l}\text { North Fork MINTON CREEK } \\
\text { TAPS A/G; LWC }\end{array}$ & GR & jafemrapSSSSSSSSSSSSnode & $\begin{array}{l}2686406 \\
2686466 \\
1039588\end{array}$ & $\begin{array}{l}\mathrm{F} \\
06 \mathrm{~S} \\
07 \mathrm{E} \\
14\end{array}$ & $\mathrm{~F}^{\mathrm{B}}$ \\
\hline $\begin{array}{c}508.85 \\
51\end{array}$ & $\begin{array}{l}\text { North Fork MINTON CREEK } \\
\text { TAPS A/G; LWC }\end{array}$ & GR & jafemrapSSSSSSSSSSSSnode & $\begin{array}{l}2686700 \\
2686760 \\
1039301\end{array}$ & $\begin{array}{l}\mathrm{F} \\
06 \mathrm{~S} \\
07 \mathrm{E} \\
14\end{array}$ & B \\
\hline $\begin{array}{c}509.20 \\
51\end{array}$ & $\begin{array}{l}\text { North Fork MINTON CREEK } \\
\text { TAPS A/G; LWC }\end{array}$ & GR & jafemrapSSSSSSSSSSSSnode & $\begin{array}{l}2688540 \\
2688608 \\
1037413\end{array}$ & $\begin{array}{l}F \\
06 S \\
07 E \\
14\end{array}$ & $\mathrm{~F}^{\mathrm{B}}$ \\
\hline $\begin{array}{c}509.72 \\
51\end{array}$ & $\begin{array}{l}\text { North Fork MINTON CREEK } \\
\text { TAPS A/G; LWC }\end{array}$ & $\mathrm{GR}$ & jafemrapSSSSSSSSSSSSnode & $\begin{array}{l}2691314 \\
2691372 \\
1034600\end{array}$ & $\begin{array}{l}\mathrm{F} \\
06 \mathrm{~S} \\
07 \mathrm{E} \\
13 \\
\end{array}$ & $\begin{array}{ll}\mathrm{AB} & \mathrm{E} \\
\mathrm{F} & \end{array}$ \\
\hline
\end{tabular}


USDOI BLM PMO OPEN FILE REPORT - TAPS FISH STREAMS

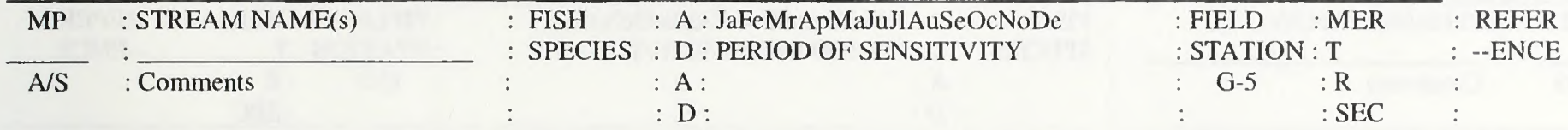

\begin{tabular}{|c|c|c|c|c|c|c|}
\hline $\begin{array}{c}509.84 \\
51\end{array}$ & $\begin{array}{l}\text { South Fork MINTON CREEK } \\
\text { TAPS A/G; LWC }\end{array}$ & $\overline{G R}$ & jafemrapSSSSSSSSSSSSnode & $\begin{array}{l}2691903 \\
2691956 \\
1034300\end{array}$ & $\begin{array}{l}\mathrm{F} \\
06 \mathrm{~S} \\
07 \mathrm{E} \\
13\end{array}$ & $\begin{array}{ll}\mathrm{AB} & \mathrm{E} \\
\mathrm{F} & \end{array}$ \\
\hline $\begin{array}{c}* 509.8 \\
51\end{array}$ & $\begin{array}{l}\text { South Fork MINTON CREEK } \\
\text { TAPS A/G; TAPS DOES NOT } \\
\text { CROSS }\end{array}$ & GR & jafemrapSSSSSSSSSSSSnode & $\begin{array}{l}\text { NO } \\
\text { TAPS } \\
\text { XING } \\
1034012\end{array}$ & $\begin{array}{l}\mathrm{F} \\
06 \mathrm{~S} \\
07 \mathrm{E} \\
13\end{array}$ & $\mathrm{~F}$ \\
\hline $\begin{array}{c}510.32 \\
51\end{array}$ & $\begin{array}{l}\text { South Fork MINTON CREEK } \\
\text { TAPS A/G; LWC }\end{array}$ & GR & jafemrapSSSSSSSSSSSSnode & $\begin{array}{l}2694468 \\
2694527 \\
1031800\end{array}$ & $\begin{array}{l}\mathrm{F} \\
06 \mathrm{~S} \\
07 \mathrm{E} \\
13\end{array}$ & $\begin{array}{ll}\mathrm{AB} & \mathrm{E} \\
\mathrm{F} & \end{array}$ \\
\hline $\begin{array}{c}510.54 \\
51\end{array}$ & $\begin{array}{l}\text { South Fork MINTON CREEK } \\
\text { TAPS A/G; LWC }\end{array}$ & GR & jafemrapSSSSSSSSSSSSnode & $\begin{array}{l}2695613 \\
2695673 \\
1030560\end{array}$ & $\begin{array}{l}\mathrm{F} \\
06 \mathrm{~S} \\
07 \mathrm{E} \\
24 \\
\end{array}$ & $\begin{array}{l}\mathrm{AB} \\
\mathrm{F}\end{array}$ \\
\hline $\begin{array}{c}511.45 \\
51\end{array}$ & $\begin{array}{l}\text { South Fork MINTON CREEK } \\
\text { TAPS A/G; LWC }\end{array}$ & GR & jsfemrapSSSSSSSSSSSSnode & $\begin{array}{l}2700423 \\
2700483 \\
1025812\end{array}$ & $\begin{array}{l}\mathrm{F} \\
06 \mathrm{~S} \\
08 \mathrm{E} \\
19 \\
\end{array}$ & $\mathrm{~F}^{\mathrm{B}}$ \\
\hline $\begin{array}{c}511.68 \\
51\end{array}$ & $\begin{array}{l}\text { South Fork MINTON CREEK } \\
\text { TAPS A/G; LWC }\end{array}$ & GR & jafemrapSSSSSSSSSSSSnode & $\begin{array}{l}2701624 \\
2701684 \\
1024406\end{array}$ & $\begin{array}{l}\mathrm{F} \\
06 \mathrm{~S} \\
08 \mathrm{E} \\
19 \\
\end{array}$ & $\mathrm{~F}^{\mathrm{B}}$ \\
\hline $\begin{array}{c}512.30 \\
51\end{array}$ & $\begin{array}{l}\text { South Fork MINTON CREEK } \\
\text { TAPS A/G; LWC }\end{array}$ & GR & $\begin{array}{l}\text { jafemrapSSSSSSSSSSSSnode } \\
\end{array}$ & $\begin{array}{l}2704924 \\
2704981 \\
1021480\end{array}$ & $\begin{array}{l}\mathrm{F} \\
06 \mathrm{~S} \\
08 \mathrm{E} \\
29 \\
\end{array}$ & $\mathrm{~F}^{\mathrm{B}}$ \\
\hline $\begin{array}{c}518.89 \\
49\end{array}$ & $\begin{array}{l}\text { West Branch KEYSTONE } \\
\text { CREEK } \\
\text { TAPS A/G; CMP }\end{array}$ & $\mathrm{GR} ; \mathrm{BB}$ & jafemrapSSSSSSSSSSSSnode & $\begin{array}{l}2739729 \\
2739789 \\
9867+50 \\
9862+00 \\
\end{array}$ & $\begin{array}{l}\mathrm{F} \\
07 \mathrm{~S} \\
08 \mathrm{E} \\
23 \\
\end{array}$ & $\begin{array}{l}A \\
F\end{array}$ \\
\hline $\begin{array}{c}520.08 \\
49\end{array}$ & $\begin{array}{l}\text { ROSA CREEK } \\
\text { TAPS A/G; CMP }\end{array}$ & GR & jafemrapSSSSSSSSSSSSnode & $\begin{array}{l}2746040 \\
2746100 \\
9800+35\end{array}$ & $\begin{array}{l}\mathrm{F} \\
07 \mathrm{~S} \\
08 \mathrm{E} \\
25 \\
\end{array}$ & $\begin{array}{ll}\mathrm{AB} & \mathrm{E} \\
\mathrm{F} & \end{array}$ \\
\hline $\begin{array}{c}520.31 \\
49\end{array}$ & $\begin{array}{l}\text { SHAW CREEK } \\
\text { TAPS A/G; BRIDGE }\end{array}$ & $\begin{array}{l}\text { BB;CN; } \\
\text { DS;GR; } \\
\text { HW;LC; } \\
\text { LS;NP; } \\
\text { RW;SB? } \\
\text { SS } \\
\end{array}$ & $\begin{array}{l:l}\mathrm{Y} & \mathrm{CCCCCCCCCCCCCCCCCCCCCCC} \\
\mathrm{E} & \mathrm{C} \\
S & \end{array}$ & $\begin{array}{l}2747200 \\
2747350 \\
9788+89\end{array}$ & $\begin{array}{l}\mathrm{F} \\
07 \mathrm{~S} \\
08 \mathrm{E} \\
36\end{array}$ & $\begin{array}{ll}\mathrm{AB} & \mathrm{E} \\
\mathrm{F} & \end{array}$ \\
\hline OMS 49 & $2 \mathrm{~N} \quad$ (Four-Nine CK) & & & & B & \\
\hline $\begin{array}{c}531.15 \\
531.32 \\
47\end{array}$ & $\begin{array}{l}\text { TANANA RIVER } \\
\text { TAPS A/G; BLOCKPOINT }\end{array}$ & $\begin{array}{l}\text { BB;BW; } \\
\text { CN;DS; } \\
\text { DV?GR; } \\
\text { HW;IN: } \\
\text { KS;LC; } \\
\text { LS;NP; } \\
\text { RW;SS }\end{array}$ & $\begin{array}{l:l}\mathrm{CCCCCCCCCCCCCCCCCCCCCCC} \\
\mathrm{E}: \mathrm{C} \\
\mathrm{S}\end{array}$ & $\begin{array}{l}2804500 \\
2805400 \\
9218+72 \\
9209+98\end{array}$ & $\begin{array}{l}\mathrm{F} \\
09 \mathrm{~S} \\
10 \mathrm{E} \\
06 \& \\
07\end{array}$ & $\begin{array}{ll}A B & E \\
F\end{array}$ \\
\hline
\end{tabular}


USDOI BLM PMO OPEN FILE REPORT - TAPS FISH STREAMS

\begin{tabular}{llll}
\hline MP & $:$ STREAM NAME(s) & $:$ FlSH & $:$ A : JaFeMrApMaJuJlAuSeOcNoDe \\
& $:$ SPECIES & $:$ D : PERIOD OF SENSITIVITY \\
& $:$ Comments & $:$ & $:$ A : \\
& & $:$ & $: D:$
\end{tabular}

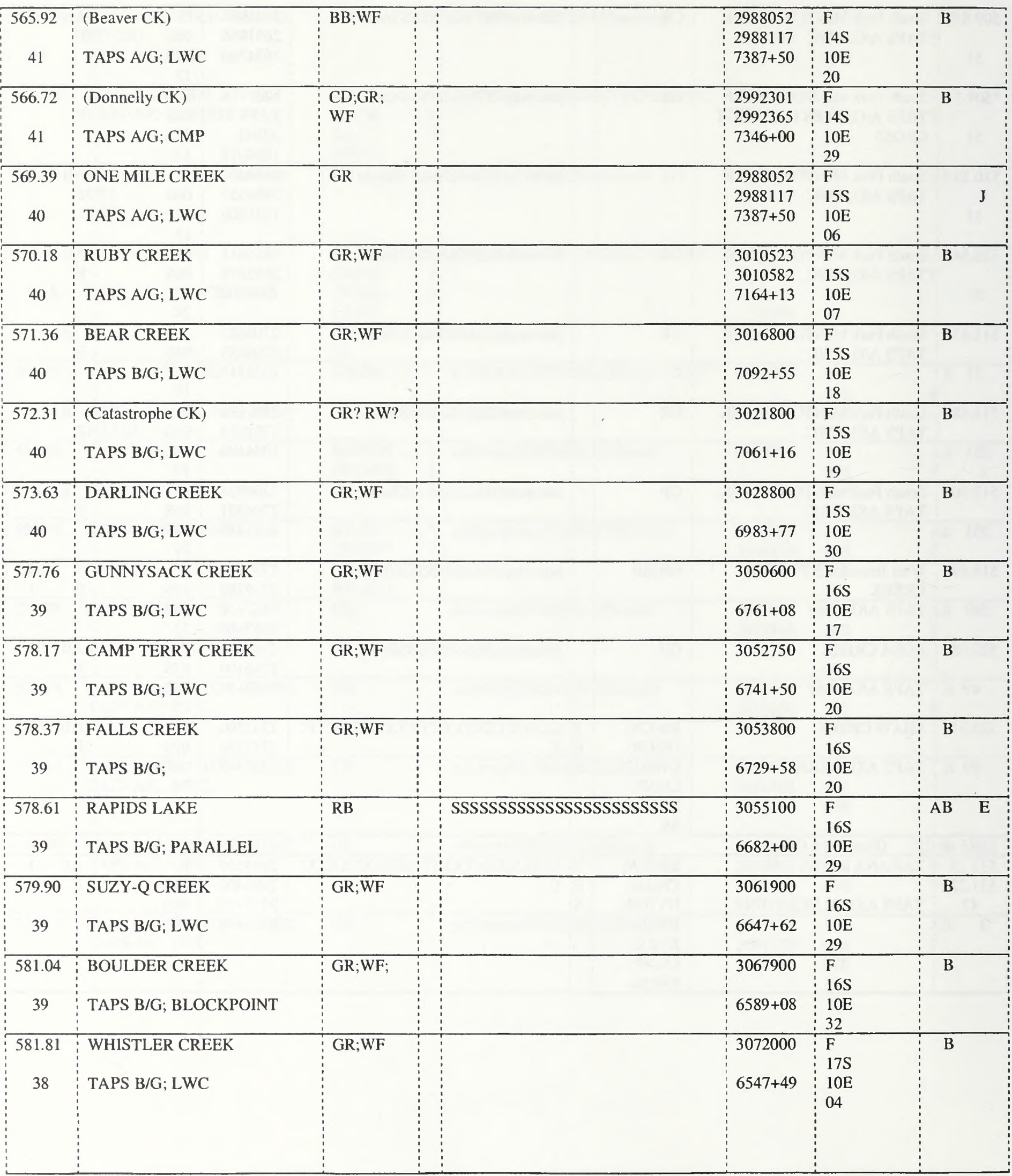

07/30/03 PAGE 32 of 41

:STATION : T : --ENCE

: G-5 :R

: SEC
$:$ FIELD : MER : REFER 
USDOI BLM PMO OPEN FILE REPORT - TAPS FISH STREAMS

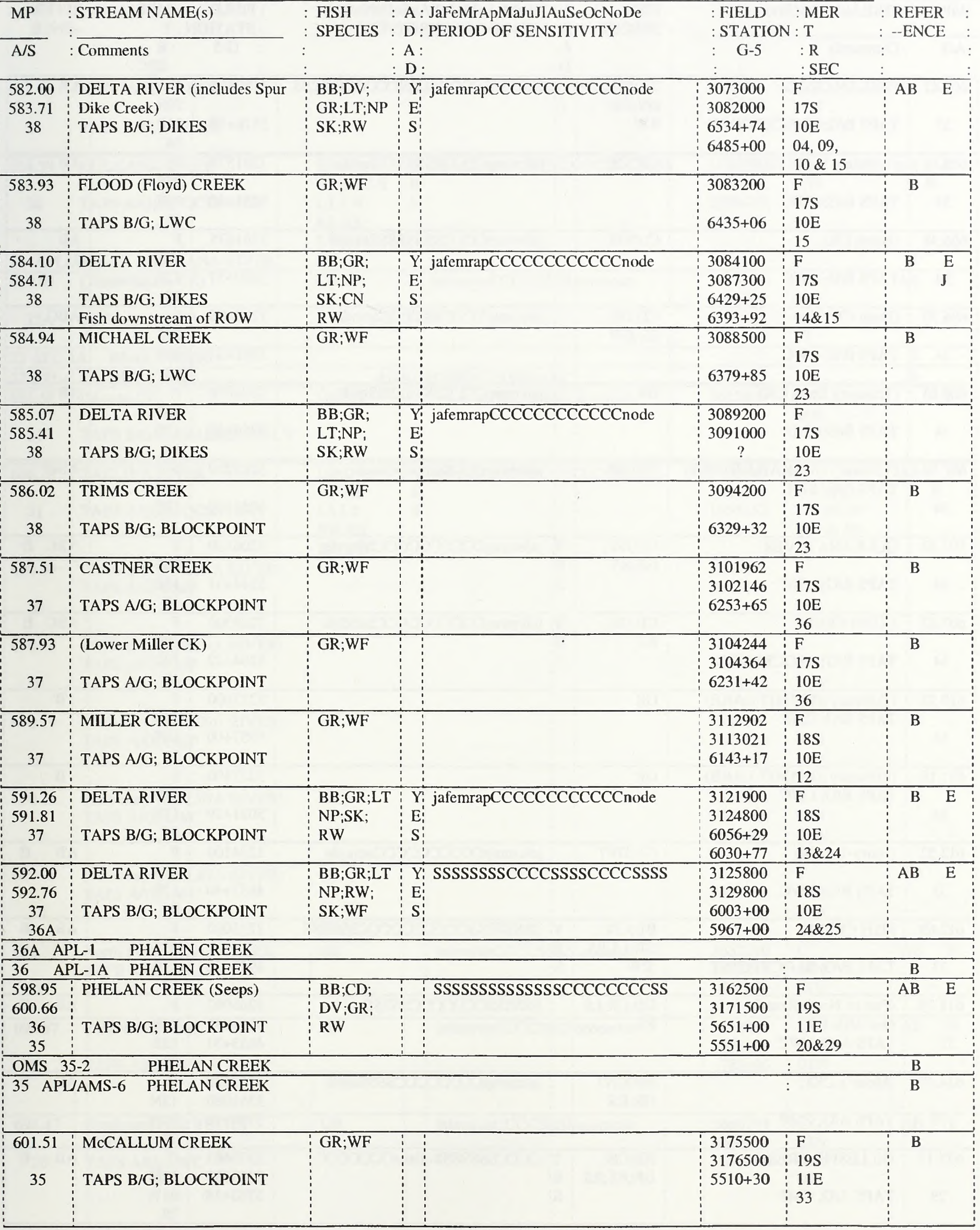


USDOI BLM PMO OPEN FILE REPORT - TAPS FISH STREAMS

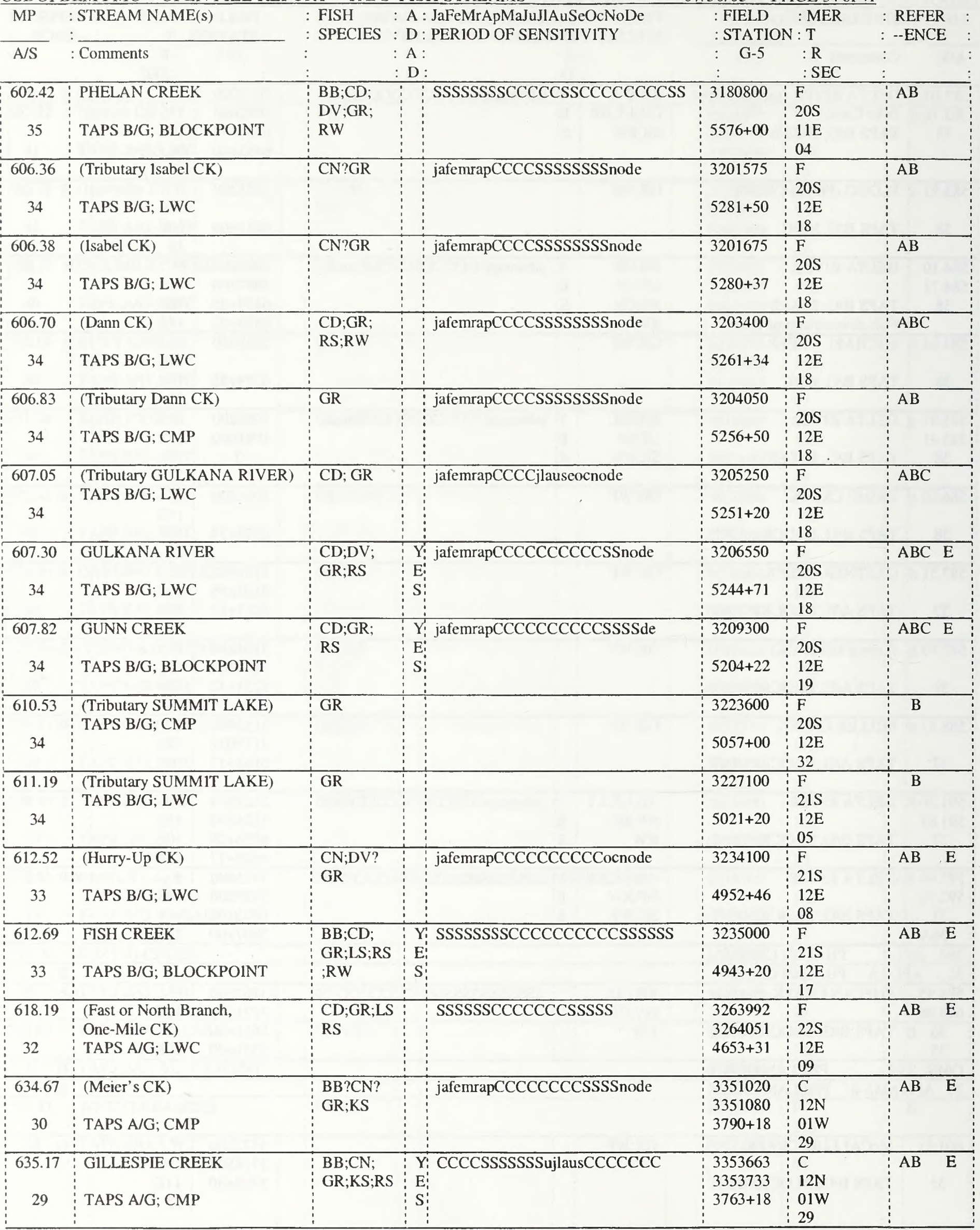


USDOI BLM PMO OPEN FILE REPORT - TAPS FISH STREAMS

\begin{tabular}{|c|c|c|c|c|c|c|}
\hline MP & : STREAM NAME(s) & $\begin{array}{l}\text { FISH } \\
\text { SPECIES : }\end{array}$ & $\begin{array}{l}\text { A : JaFeMrApMaJuJlAuSeOcNoDe } \\
\text { D : PERIOD OF SENSITIVITY }\end{array}$ & $\begin{array}{l}: \text { FIELD } \\
: \text { STATION }\end{array}$ & & $\begin{array}{l}\text { REFER } \\
-- \text { ENCE }\end{array}$ \\
\hline $\mathrm{A} / \mathrm{S}$ & : Comments & & & $: \quad$ G-5 & $\begin{array}{l}: \mathrm{R} \\
: \mathrm{SEC}\end{array}$ & \\
\hline $\begin{array}{c}642.48 \\
28\end{array}$ & $\begin{array}{l}\text { HAGGARD CREEK } \\
\text { TAPS A/G; BRIDGE }\end{array}$ & GR;LS & jafemrapCCCCSSSSSSSSnode & $\begin{array}{l}3392235 \\
3392305 \\
3394+49\end{array}$ & $\begin{array}{l}\mathrm{C} \\
11 \mathrm{~N} \\
01 \mathrm{~W} \\
32\end{array}$ & $\mathrm{AB} \quad \mathrm{E}$ \\
\hline $\begin{array}{c}654.39 \\
26\end{array}$ & $\begin{array}{l}\text { GULKANA RIVER } \\
\text { TAPS A/G; BLOCKPOINT }\end{array}$ & $\begin{array}{l}\text { BB;CD; } \\
\text { GR;KS;LS } \\
\text { LT;LW; } \\
\text { RB;RS; } \\
\text { RW;SH } \\
\end{array}$ & \begin{tabular}{c:c} 
& SSSSSSSSCCCCCCCCCCCCSSSS \\
\hdashline & \\
$S$ & \\
\end{tabular} & $\begin{array}{l}3454931 \\
3455411 \\
2796+75 \\
2794+17\end{array}$ & $\begin{array}{l}\mathrm{C} \\
09 \mathrm{~N} \\
02 \mathrm{~W} \\
26\end{array}$ & $\mathrm{AB}_{\text {HI }} \mathrm{E}$ \\
\hline $26 \mathrm{APL}$ & AMS-2 GULKANA RIVER & & & & & B \\
\hline $\begin{array}{r}659.69 \\
25 \mathrm{~A}\end{array}$ & $\begin{array}{l}\text { (Three-Sisters CK) } \\
\text { TAPS A/G; CMP }\end{array}$ & CD;GR & jafemrapCCCCSSSSseocnode & $\begin{array}{l}3483149 \\
3483209 \\
2473+42\end{array}$ & $\begin{array}{l}\mathrm{C} \\
08 \mathrm{~N} \\
02 \mathrm{~W} \\
23 \\
\end{array}$ & $\mathrm{AB} \quad \mathrm{E}$ \\
\hline$\frac{23 \mathrm{APL}-}{22 \mathrm{APL}-}$ & 1A BEAR CREEK & & & & & $\frac{B}{D}$ \\
\hline $\begin{array}{c}22 \text { APL- } \\
686.55 \\
21\end{array}$ & $\begin{array}{l}\text { DRY CREEK } \\
\text { TAOSE CK) } \\
\text { TAPS B/G; PARALLEL ONLY }\end{array}$ & GR;SK & $\begin{array}{l}\text { ADROMOUS STREAM } \\
\vdots \text { jafemrapCCCCSSSSseocnode }\end{array}$ & 3625000 & $\begin{array}{l}\mathrm{C} \\
04 \mathrm{~N} \\
02 \mathrm{~W} \\
31\end{array}$ & $\mathrm{E}$ \\
\hline $\begin{array}{c}686.78 \\
21\end{array}$ & $\begin{array}{l}\text { TAZLINA RIVER } \\
\text { TAPS AVG; BLOCKPOINT }\end{array}$ & $\begin{array}{l}\text { BB;DV; } \\
\text { GR;KS } \\
\text { LS;LT; } \\
\text { PW;RS } \\
\text { RW;SH }\end{array}$ & $\begin{array}{l}\text { YafemrSSCCCCCCCCCCCCSSSS } \\
S_{0}\end{array}$ & $\begin{array}{l}3626000 \\
3626400 \\
1055+53 \\
1051+99\end{array}$ & $\begin{array}{l}\mathrm{C} \\
4 \mathrm{~N} \& 3 \mathrm{~N} \\
2 \mathrm{~W} \& 2 \mathrm{~W} \\
31 \& 06\end{array}$ & ${ }_{\mathrm{H}}^{\mathrm{AB}} \mathrm{E}$ \\
\hline $\begin{array}{c}690.09 \\
20\end{array}$ & $\begin{array}{l}\text { (Tributary TAZLINA RIVER) } \\
\text { TAPS A/G; CMP }\end{array}$ & $\overline{\mathrm{GR}}$ & & $\begin{array}{r}3643628 \\
3643688 \\
881+87\end{array}$ & $\begin{array}{l}\mathrm{C} \\
03 \mathrm{~N} \\
01 \mathrm{~W} \\
20 \\
\end{array}$ & $\bar{B}$ \\
\hline $\begin{array}{c}690.16 \\
20\end{array}$ & $\begin{array}{l}\text { (Tributary TAZLINA RIVER) } \\
\text { TAPS A/G; CMP }\end{array}$ & GR & & $\begin{array}{r}3643987 \\
3644054 \\
878+12\end{array}$ & $\begin{array}{l}\mathrm{C} \\
03 \mathrm{~N} \\
01 \mathrm{~W} \\
20\end{array}$ & B \\
\hline $\begin{array}{c}690.19 \\
20\end{array}$ & $\begin{array}{l}\text { (Tributary TAZLINA RIVER) } \\
\text { TAPS A/G; CMP }\end{array}$ & GR & & $\begin{array}{r}3644166 \\
1644226 \\
876+52\end{array}$ & $\begin{array}{l}\mathrm{C} \\
03 \mathrm{~N} \\
01 \mathrm{~W} \\
20\end{array}$ & B \\
\hline $\begin{array}{c}690.23 \\
20\end{array}$ & $\begin{array}{l}\text { (Tributary TAZLINA RIVER) } \\
\text { TAPS A/G; LWC }\end{array}$ & GR & & $\begin{array}{r}3644406 \\
3644466 \\
874+01\end{array}$ & $\begin{array}{l}\mathrm{C} \\
03 \mathrm{~N} \\
01 \mathrm{~W} \\
20\end{array}$ & B \\
\hline $\begin{array}{c}690.28 \\
20\end{array}$ & $\begin{array}{l}\text { (Tributary TAZLINA RIVER) } \\
\text { TAPS A/G; LWC }\end{array}$ & GR & & $\begin{array}{r}3644645 \\
3644713 \\
871+63\end{array}$ & $\begin{array}{l}\mathrm{C} \\
03 \mathrm{~N} \\
01 \mathrm{~W} \\
20 \\
\end{array}$ & B \\
\hline $\begin{array}{c}691.71 \\
20\end{array}$ & $\begin{array}{l}\text { North Fork YETNA CREEK } \\
\text { TAPS N/G; LWC }\end{array}$ & GR & jafemrapCCCCSSSSseocnode & $\begin{array}{r}3652210 \\
3652270 \\
797+20\end{array}$ & $\begin{array}{l}\mathrm{C} \\
03 \mathrm{~N} \\
01 \mathrm{~W} \\
28\end{array}$ & $\overline{A B}$ \\
\hline $\begin{array}{c}691.87 \\
20\end{array}$ & $\begin{array}{l}\text { YETNA CREEK } \\
\text { TAPS A/G; LWC }\end{array}$ & GR & jafemrapCCCCSSSSseocnode & $\begin{array}{r}3653057 \\
3653110 \\
788+00\end{array}$ & $\begin{array}{l}\mathrm{C} \\
03 \mathrm{~N} \\
01 \mathrm{~W} \\
28\end{array}$ & $\overline{\mathrm{AB}}$ \\
\hline $\begin{array}{c}694.47 \\
20\end{array}$ & $\begin{array}{l}\text { Southwest Fork YETNA } \\
\text { CREEK } \\
\text { TAPS A/G; LWC }\end{array}$ & GR & jafemrapCCCCSSSSseocnode & $\begin{array}{r}3666794 \\
3666855 \\
650+00\end{array}$ & $\begin{array}{l}\mathrm{C} \\
02 \mathrm{~N} \\
01 \mathrm{~W} \\
03\end{array}$ & $\mathrm{AB}$ \\
\hline
\end{tabular}


USDOI BLM PMO OPEN FILE REPORT - TAPS FISH STREAMS

\begin{tabular}{|c|c|c|c|c|c|c|}
\hline MP & STREAM NAME(s) & $\begin{array}{l}\text { FISH } \\
: \text { SPECIES : }\end{array}$ & $\begin{array}{l}\text { A : JaFeMrApMaJuJlAuSeOcNoDe } \\
\text { D : PERIOD OF SENSITIVITY }\end{array}$ & $\begin{array}{l}\text { :FIELD } \\
\text { : STATION }\end{array}$ & $\begin{array}{l}: \text { MER } \\
: \mathrm{T}\end{array}$ & $\begin{array}{l}\text { REFER } \\
\text {--ENCE }\end{array}$ \\
\hline $\mathrm{A} / \mathrm{S}$ & : Comments & . & $\begin{array}{l}\text { A : } \\
\text { D : }\end{array}$ & $\begin{array}{ll}: & \text { G-5 } \\
: & \end{array}$ & $\begin{array}{l}: \mathrm{R} \\
: \mathrm{SEC}\end{array}$ & \\
\hline $\begin{array}{c}697.33 \\
19\end{array}$ & $\begin{array}{l}\text { KLUTINA RIVER } \\
\text { TAPS B/G; BLOCKPOINT }\end{array}$ & $\begin{array}{l}\text { BB;DV; } \\
\text { GR;KS;LT } \\
\text { LW;PW; } \\
\text { RS;SH;SS }\end{array}$ & $\begin{array}{l}Y_{1} \text { jafemrSSCCCCCCCCCCSSSSde } \\
E_{0}^{1} \\
S:\end{array}$ & $\begin{array}{r}3681800 \\
3682000 \\
502+96 \\
501+34 \\
\end{array}$ & $\begin{array}{l}\mathrm{C} \\
02 \mathrm{~N} \\
01 \mathrm{~W} \\
13\end{array}$ & ${ }_{H}^{\mathrm{AB}}$ \\
\hline $\begin{array}{c}706.68 \\
18\end{array}$ & $\begin{array}{l}\text { WILLOW CREEK } \\
\text { TAPS A/G; BLOCKPOINT }\end{array}$ & GR & $\begin{array}{l:c}\text { jafemrapCCCCSSSseocnode } \\
\text { ind }\end{array}$ & $\begin{array}{l}3731242 \\
3731302 \\
1483+55\end{array}$ & $\begin{array}{l}\mathrm{C} \\
01 \mathrm{~N} \\
01 \mathrm{E} \\
33\end{array}$ & $\mathrm{AB}$ \\
\hline 712.25 & $\begin{array}{l}\text { ROCK CREEK } \\
\text { TAPS A/G; LWC }\end{array}$ & GR & jafemrapCCCCSSSSseocnode & $\begin{array}{l}3760652 \\
3760723 \\
1216+41\end{array}$ & $\begin{array}{l}\mathrm{C} \\
01 \mathrm{~S} \\
01 \mathrm{E} \\
26 \\
\end{array}$ & $\overline{\mathrm{AB}}$ \\
\hline $\begin{array}{c}717.28 \\
16\end{array}$ & $\begin{array}{l}\text { SQUIRREL CREEK } \\
\text { TAPS A/G; LWC }\end{array}$ & $\begin{array}{l}\text { CD;DV; } \\
\text { GR;KS } \\
\text { SS }\end{array}$ & $\begin{array}{cc} & \text { SSSSSSapSSCCCCCCCCCCSSSS } \\
E_{1} & \\
S & \\
S & \end{array}$ & $\begin{array}{r}3787207 \\
3787277 \\
951+38\end{array}$ & $\begin{array}{l}\mathrm{C} \\
02 \mathrm{~S} \\
01 \mathrm{E} \\
15 \\
\end{array}$ & $\begin{array}{ll}\mathrm{AB} & \mathrm{E} \\
& \mathrm{J}\end{array}$ \\
\hline $\begin{array}{c}720.95 \\
15\end{array}$ & $\begin{array}{l}\text { (Tributary TONSINA RIVER) } \\
\text { TAPS B/G; LWC }\end{array}$ & $\overline{G R}$ & -7 & $\begin{array}{r}3806600 \\
757+40\end{array}$ & $\begin{array}{l}\mathrm{C} \\
03 \mathrm{~S} \\
01 \mathrm{E} \\
03 \\
\end{array}$ & B \\
\hline $\begin{array}{c}723.89 \\
15\end{array}$ & $\begin{array}{l}\text { TONSINA RIVER } \\
\text { TAPS B/G; LWC }\end{array}$ & $\begin{array}{l}\mathrm{BB} ; \mathrm{CD} ; \\
\mathrm{DV} ; \mathrm{GR} ; \\
\mathrm{KS} ; \mathrm{LT} ; \mathrm{P} \\
\text { W } \\
\text { RS;RW; } \\
\text { SH;SS }\end{array}$ & $\begin{array}{l}\text { Y jafemrSSSSCCCCCCCCCCSSde } \\
\mathrm{E}_{0}\end{array}$ & $\begin{array}{l}3822125 \\
605+20 \\
603+70\end{array}$ & $\begin{array}{l}\mathrm{C} \\
03 \mathrm{~S} \\
01 \mathrm{E} \\
21\end{array}$ & ${ }_{\mathrm{H}}^{\mathrm{AB}} \mathrm{E}$ \\
\hline $\begin{array}{c}724.01 \\
15\end{array}$ & $\begin{array}{l}\text { TONSINA RIVER } \\
\text { TAPS B/G; BRIDGE }\end{array}$ & $\begin{array}{l}\text { BB;CD; } \\
\text { DV;GR; } \\
\text { KS;LT;P } \\
\text { WRS;RW; } \\
\text { SH;SS } \\
\end{array}$ & $\begin{array}{l}\text { Y: jafemrSSSSCCCCCCCCCCSSde } \\
\text { E: } \\
\text { S: }\end{array}$ & $\begin{array}{r}3822735 \\
3822860 \\
599+15 \\
598+00\end{array}$ & $\begin{array}{l}\mathrm{C} \\
03 \mathrm{~S} \\
01 \mathrm{E} \\
21\end{array}$ & ${ }_{H}^{\mathrm{AB}} \mathrm{E}$ \\
\hline $\begin{array}{c}730.17 \\
14\end{array}$ & $\begin{array}{l}\text { (Silver Spawning CK) } \\
\text { TAPS B/G; LWC }\end{array}$ & DV;SS & jafemrapmajujlauSSSSnode & $\begin{array}{r}3855300 \\
329+70\end{array}$ & $\begin{array}{l}\mathrm{C} \\
04 \mathrm{~S} \\
01 \mathrm{E} \\
16 \\
\end{array}$ & B \\
\hline $\begin{array}{c}730.25 \\
13\end{array}$ & $\begin{array}{l}\text { (Silver Spawning CK) } \\
\text { TAPS B/G; LWC }\end{array}$ & $\mathrm{CD} ; \mathrm{DV} ; \mathrm{SS}$ & jafemrapmajuSSSSSSSSnode & $\begin{array}{r}3855700 \\
324+65\end{array}$ & $\begin{array}{l}C \\
04 S \\
01 E \\
16\end{array}$ & B \\
\hline $\begin{array}{c}730.36 \\
13\end{array}$ & $\begin{array}{l}\text { (North Fork Crack CK) } \\
\text { TAPS B/G; LWC }\end{array}$ & $\mathrm{CD} ; \mathrm{SS}$ & & $\begin{array}{r}3856275 \\
318+40\end{array}$ & $\begin{array}{l}\mathrm{C} \\
04 \mathrm{~S} \\
01 \mathrm{E} \\
16 \\
\end{array}$ & B \\
\hline $\begin{array}{c}730.47 \\
13\end{array}$ & $\begin{array}{l}\text { (South Fork Crack CK) } \\
\text { TAPS B/G; LWC }\end{array}$ & $\mathrm{CD} ; \mathrm{DV} ; \mathrm{SS}$ & & $\begin{array}{r}3856900 \\
311+65\end{array}$ & $\begin{array}{l}\mathrm{C} \\
04 \mathrm{~S} \\
01 \mathrm{E} \\
21\end{array}$ & B $\quad$ E \\
\hline $\begin{array}{c}730.66 \\
13\end{array}$ & $\begin{array}{l}\text { (Little Tonsina Flats) } \\
\text { TAPS B.G; CMP }\end{array}$ & $\begin{array}{l}\mathrm{CN} ; \mathrm{DV} \\
\mathrm{GR} ; \mathrm{SS}\end{array}$ & jafemrapmajujISSSSocnode & $\begin{array}{r}3857900 \\
302+65\end{array}$ & $\begin{array}{l}\mathrm{C} \\
04 \mathrm{~S} \\
01 \mathrm{E} \\
21 \\
\end{array}$ & $\mathrm{BC} \quad \mathrm{E}$ \\
\hline $\begin{array}{c}730.72 \\
13\end{array}$ & $\begin{array}{l}\text { (Little Tonsina Flats) } \\
\text { TAPS B/G; LWC }\end{array}$ & $\begin{array}{l}\mathrm{CN} ; \mathrm{DV} \\
\mathrm{GR} ; \mathrm{SS}\end{array}$ & jafemrapmajujlSSSSocnode & $\begin{array}{r}3858200 \\
298+45\end{array}$ & $\begin{array}{l}\mathrm{C} \\
04 \mathrm{~S} \\
01 \mathrm{E} \\
21 \\
\end{array}$ & $\mathrm{BC} \quad \mathrm{E}$ \\
\hline $\begin{array}{c}730.78 \\
13\end{array}$ & $\begin{array}{l}\text { (Little Tonsina Flats) } \\
\text { TAPS B/G; LWC }\end{array}$ & $\begin{array}{l}\text { CN;DV; } \\
\text { GR;SS }\end{array}$ & jafemrapmajujlSSSSocnode & $\begin{array}{r}3858500 \\
294+20\end{array}$ & $\begin{array}{l}\mathrm{C} \\
04 \mathrm{~S} \\
01 \mathrm{E} \\
21\end{array}$ & $\mathrm{BC} \mathrm{E}$ \\
\hline
\end{tabular}


USDOI BLM PMO OPEN FILE REPORT - TAPS FISH STREAMS

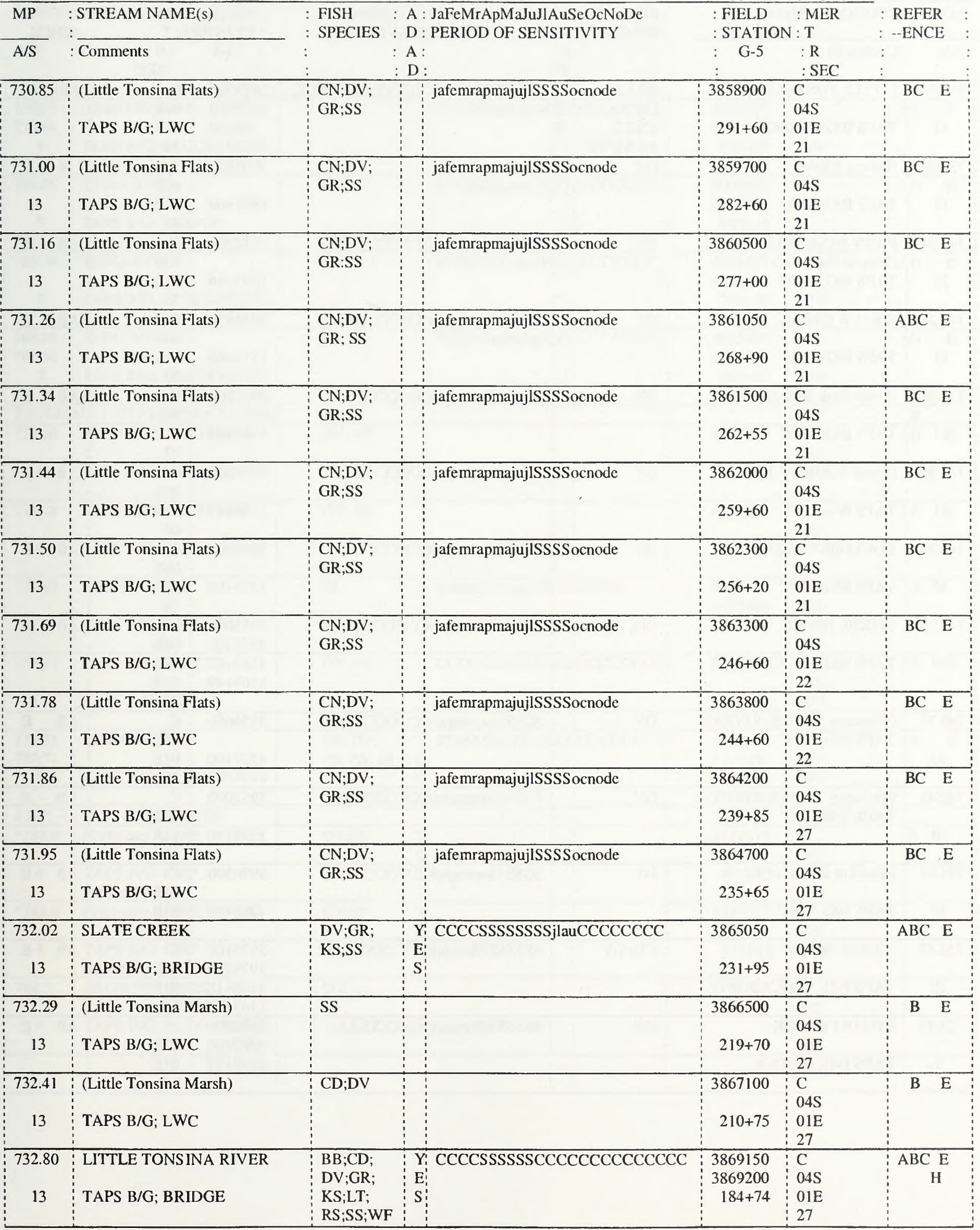


USDOI BLM PMO OPEN FILE REPORT - TAPS FISH STREAMS

\begin{tabular}{|c|c|c|c|c|c|c|}
\hline MP & STREAM NAME(s) & $\begin{array}{l}\text { FISH } \\
\text { SPECIES }\end{array}$ & $\begin{array}{l}\text { A : JaFeMrApMaJuJlAuSeOcNoDe } \\
\text { D : PERIOD OF SENSITIVITY }\end{array}$ & $\begin{array}{l}: \text { F1ELD } \\
: \text { STATIC }\end{array}$ & $\begin{array}{l}: \text { MER } \\
: T\end{array}$ & $\begin{array}{l}\text { REFER } \\
\text {--ENCE }\end{array}$ \\
\hline $\mathrm{A} / \mathrm{S}$ & : Comments & & $\begin{array}{l}\text { A : } \\
\text { D: }\end{array}$ & $\begin{array}{ll}: & \mathrm{G}-5 \\
\end{array}$ & $\begin{array}{l}: \mathrm{R} \\
: \text { SEC }\end{array}$ & \\
\hline $\begin{array}{c}734.66 \\
13\end{array}$ & $\begin{array}{l}\text { LITTLE TONSINA RIVER } \\
\text { TAPS B/G; BRIDGE }\end{array}$ & $\begin{array}{l}\mathrm{BB} ; \mathrm{CD} \\
\mathrm{DV} ; \mathrm{GR} ; \\
\mathrm{KS} ; \mathrm{LT} ; \\
\mathrm{RS} ; \mathrm{SS} ; \mathrm{WF}\end{array}$ & 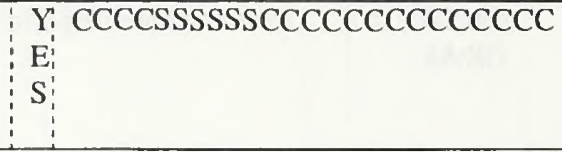 & $\begin{array}{r}3879000 \\
3879050 \\
86+56\end{array}$ & $\begin{array}{l}\mathrm{C} \\
04 \mathrm{~S} \\
01 \mathrm{E} \\
36\end{array}$ & $\begin{array}{c}\mathrm{ABC} E \\
\mathrm{H}\end{array}$ \\
\hline $\begin{array}{c}739.26 \\
12\end{array}$ & $\begin{array}{l}\text { (Quarry CK) } \\
\text { TAPS B/G; LWC }\end{array}$ & DV & & $\begin{array}{l}3903300 \\
1893+00\end{array}$ & $\begin{array}{l}\mathrm{C} \\
05 \mathrm{~S} \\
01 \mathrm{E} \\
23 \\
\end{array}$ & $\bar{B}$ \\
\hline $\begin{array}{c}741.04 \\
12\end{array}$ & $\begin{array}{l}\text { F1FTY-NINE MILE CREEK } \\
\text { (Wagon Point CK) } \\
\text { TAPS B/G; LWC }\end{array}$ & DV & SSSSSSapmajujlCCCCCCCCCC & $\begin{array}{l}3912700 \\
1797+16\end{array}$ & $\begin{array}{l}\mathrm{C} \\
05 \mathrm{~S} \\
01 \mathrm{E} \\
26 \\
\end{array}$ & $\mathrm{AB} \quad \mathrm{E}$ \\
\hline $\begin{array}{c}745.26 \\
11\end{array}$ & $\begin{array}{l}\text { SQUAW CREEK } \\
\text { TAPS B/G; LWC }\end{array}$ & DV & $\begin{array}{l}\text { SSSSSSapmajujlCCCCCCCCCC } \\
\text {. }\end{array}$ & $\begin{array}{l}3934950 \\
1571+60\end{array}$ & $\begin{array}{l}\mathrm{C} \\
06 \mathrm{~S} \\
01 \mathrm{E} \\
08\end{array}$ & $\overline{\mathrm{AB}}$ \\
\hline $\begin{array}{c}745.68 \\
11\end{array}$ & $\begin{array}{l}\text { (South Fork SQUAW CK) } \\
\text { TAPS B/G; LWC }\end{array}$ & DV & SSSSSSapmajujlCCCCCCCCCC & $\begin{array}{l}3937200 \\
1561+44\end{array}$ & $\begin{array}{l}\mathrm{C} \\
06 \mathrm{~S} \\
01 \mathrm{E} \\
07\end{array}$ & $\begin{array}{ll}\mathrm{AB} & \mathrm{E}\end{array}$ \\
\hline $\begin{array}{c}745.98 \\
11\end{array}$ & $\begin{array}{l}\text { (South SQUAW CK) } \\
\text { TAPS B/G; LWC }\end{array}$ & $\overline{\mathrm{DV}}$ & SSSSSSapmajujlCCCCCCCCCC & $\begin{array}{l}3938800 \\
1548+25\end{array}$ & $\begin{array}{l}\mathrm{C} \\
06 \mathrm{~S} \\
01 \mathrm{E} \\
07 \\
\end{array}$ & B E \\
\hline $\begin{array}{c}748.07 \\
10\end{array}$ & $\begin{array}{l}\text { BOULDER CREEK } \\
\text { TAPS B/G; LWC }\end{array}$ & $\overline{D V}$ & SSSSSSapmajujlCCCCCCCCCC & $\begin{array}{l}3949800 \\
1423+00\end{array}$ & $\begin{array}{l}\mathrm{C} \\
06 \mathrm{~S} \\
01 \mathrm{E} \\
24 \\
\end{array}$ & $\overline{\mathrm{AB}}$ \\
\hline $\begin{array}{c}749.07 \\
10\end{array}$ & $\begin{array}{l}\text { TIEKEL RIVER } \\
\text { TAPS B/G; BLOCKPOINT }\end{array}$ & $\mathrm{CD} ; \mathrm{DV}$ & SSSSSSSSmajujlCCCCCCCCCC & $\begin{array}{l}3955000 \\
3955200 \\
1369+02 \\
1368+49\end{array}$ & $\begin{array}{l}\mathrm{C} \\
06 \mathrm{~S} \\
01 \mathrm{E} \\
25 \& \\
30 \\
\end{array}$ & $\overline{\mathrm{AB}}$ \\
\hline $\begin{array}{c}749.37 \\
10\end{array}$ & $\begin{array}{l}\text { (Tributary TIEKEL RIVER) } \\
\text { TAPS B/G; LWC }\end{array}$ & DV & SSSSSSapmajujICCCCCCCCCC & $\begin{array}{l}3956650 \\
1357+00\end{array}$ & $\begin{array}{l}\mathrm{C} \\
06 \mathrm{~S} \\
01 \mathrm{E} \\
30 \\
\end{array}$ & $\overline{\mathrm{AB}}$ \\
\hline $\begin{array}{c}749.43 \\
10\end{array}$ & $\begin{array}{l}\text { (Tributary TIEKEL RIVER) } \\
\text { TAPS B/G; CMP }\end{array}$ & $\mathrm{DV}$ & SSSSSSapmajujlCCCCCCCCCC & $\begin{array}{l}3957000 \\
1349+80\end{array}$ & $\begin{array}{l}\mathrm{C} \\
06 \mathrm{~S} \\
01 \mathrm{E} \\
30 \\
\end{array}$ & B \\
\hline $\begin{array}{c}751.95 \\
10\end{array}$ & $\begin{array}{l}\text { (Small or Jackie's CK) } \\
\text { TAPS B/G; LWC }\end{array}$ & DV & SSSSSSapmajujlCCCCCСCCCC & $\begin{array}{l}3970300 \\
1216+00\end{array}$ & $\begin{array}{l}\mathrm{C} \\
07 \mathrm{~S} \\
01 \mathrm{E} \\
05 \\
\end{array}$ & $\overline{\mathrm{AB}}$ \\
\hline $\begin{array}{c}752.87 \\
10\end{array}$ & $\begin{array}{l}\text { TIEKEL RIVER } \\
\text { TAPS B/G; BLOCKPOINT }\end{array}$ & $\mathrm{CD} ; \mathrm{DV}$ & SSSSSSSSmajujlCCCССССССC & $\begin{array}{l}3975100 \\
3975200 \\
1168+18 \\
1167+45 \\
\end{array}$ & $\begin{array}{l}\mathrm{C} \\
07 \mathrm{~S} \\
01 \mathrm{E} \\
08 \\
\end{array}$ & $\overline{\mathrm{AB}}$ \\
\hline $\begin{array}{c}754.19 \\
9\end{array}$ & $\begin{array}{l}\text { STUART CREEK } \\
\text { TAPS B/G; BRIDGE }\end{array}$ & DV & SSSSSSSSmajujlaCCCCCCCC & $\begin{array}{l}3982000 \\
3982200 \\
1097+77\end{array}$ & $\begin{array}{l}\mathrm{C} \\
07 \mathrm{~S} \\
01 \mathrm{E} \\
18 \\
\end{array}$ & $\overline{A B}$ \\
\hline
\end{tabular}


USDOI BLM PMO OPEN FILE REPORT - TAPS FISH STREAMS

\begin{tabular}{|c|c|c|}
\hline MP & : STREAM NAME(s) & A : JaFeMrApMaJuJlAuSeOcNoDe \\
\hline $\mathrm{A} / \mathrm{S}$ & : Comments & : A : \\
\hline
\end{tabular}

07/30/03 PAGE 39 of 41

$\begin{array}{ll}\text { :FIELD : MER } & \text { : REFER } \\ : \text { STATION }: \mathrm{T} & :- \text { ENCE } \\ : \text { G-5 } \quad: \text { R } & :\end{array}$

\begin{tabular}{|c|c|c|c|c|c|c|c|}
\hline $\begin{array}{c}758.29 \\
758.64 \\
9\end{array}$ & $\begin{array}{l}\text { (Gravel Pit Pond) } \\
\text { TAPS B/G; BLOCKPOINT }\end{array}$ & DV & SSSSSSapmajujlCCCCCCCCCC & $\begin{array}{r}4003750 \\
4005640 \\
868+87 \\
863+65\end{array}$ & $\begin{array}{l}\mathrm{C} \\
07 \mathrm{~S} \\
01 \mathrm{~W} \\
34\end{array}$ & $\mathrm{AB}$ & $\mathrm{E}$ \\
\hline $\begin{array}{c}759.05 \\
9\end{array}$ & $\begin{array}{l}\text { TSINA RIVER } \\
\text { TAPS B/G; BRIDGE }\end{array}$ & DV & SSSSSSapmajujlCCCCCCCCCC & $\begin{array}{r}4007650 \\
4007900 \\
842+38\end{array}$ & $\begin{array}{l}\mathrm{C} \\
07 \mathrm{~S} \\
01 \mathrm{~W} \\
34\end{array}$ & $\mathrm{AB}$ & $\mathrm{E}$ \\
\hline $\begin{array}{c}765.38 \\
7\end{array}$ & $\begin{array}{l}\text { TSINA RIVER } \\
\text { TAPS B/G; BLOCKPOINT }\end{array}$ & DV & SSSSSSapmajujlCCCCCCCCCC & $\begin{array}{r}4041025 \\
4041325 \\
505+00 \\
502+95 \\
\end{array}$ & $\begin{array}{l}\mathrm{C} \\
08 \mathrm{~S} \\
02 \mathrm{~W} \\
03\end{array}$ & $\mathrm{AB}$ & $\mathrm{E}$ \\
\hline $\begin{array}{c}768.16 \\
768.56 \\
7\end{array}$ & $\begin{array}{l}\text { TSINA RIVER } \\
\text { TAPS B/G; BLOCKPOINT }\end{array}$ & DV & SSSSSSapmajujlCCCCCCCCCC & $\begin{array}{r}4055900 \\
4058000 \\
355+80 \\
334+58 \\
\end{array}$ & $\begin{array}{l}\mathrm{C} \\
08 \mathrm{~S} \\
02 \mathrm{~W} \\
07 \& 08 \\
\end{array}$ & $\mathrm{AB}$ & $\mathrm{E}$ \\
\hline $7 \mathrm{APUA}$ & MS-1; PTARMIGAN CREEK & & & & & $\mathrm{B}$ & \\
\hline $\begin{array}{c}774.20 \\
6\end{array}$ & $\begin{array}{l}\text { PTARMIGAN CREEK } \\
\text { TAPS B/G; LWC }\end{array}$ & DV; RB & & $\begin{array}{r}4087750 \\
34+15\end{array}$ & $\begin{array}{l}\mathrm{C} \\
08 \mathrm{~S} \\
03 \mathrm{~W} \\
27 \\
\end{array}$ & $\mathrm{AB}$ & $\mathrm{E}$ \\
\hline $\begin{array}{c}774.28 \\
6\end{array}$ & $\begin{array}{l}\text { PTARMIGAN CREEK } \\
\text { TAPS B/G; CMP }\end{array}$ & DV; RB & & $\begin{array}{r}4088200 \\
30+85\end{array}$ & $\begin{array}{l}\mathrm{C} \\
08 \mathrm{~S} \\
03 \mathrm{~W} \\
27\end{array}$ & $\overline{\mathrm{AB}}$ & $\mathrm{E}$ \\
\hline $\begin{array}{c}777.87 \\
5\end{array}$ & $\begin{array}{l}\text { SHEEP CREEK } \\
\text { TAPS B/G; LWC }\end{array}$ & SS & jafemrapmajujlSSSSSSSSde & $\begin{array}{l}4106900 \\
4107400 \\
1207+44\end{array}$ & $\begin{array}{l}\mathrm{C} \\
09 \mathrm{~S} \\
03 \mathrm{~W} \\
08\end{array}$ & $\mathrm{~B}$ & \\
\hline $\begin{array}{c}779.11 \\
5\end{array}$ & $\begin{array}{l}\text { (Seventeen Mile CK) } \\
\text { TAPS B/G; LWC }\end{array}$ & DV; SS & CCCCSSSSSSSSjlauCCCCCCCC & $\begin{array}{l}4113700 \\
1140+66\end{array}$ & $\begin{array}{l}\mathrm{C} \\
09 \mathrm{~S} \\
03 \mathrm{~W} \\
07\end{array}$ & $\mathrm{AB}$ & $\mathrm{E}$ \\
\hline $\begin{array}{l}779.38 \\
779.71 \\
5\end{array}$ & $\begin{array}{l}\text { LOWE RIVER } \\
\text { TAPS B/G; BLOCKPOINT }\end{array}$ & $\begin{array}{l}\text { DS; DV; } \\
\text { PS; RS; SS }\end{array}$ & $\begin{array}{l:l}\mathrm{Y}_{1} & \text { SSSSSSSSSSSSCCCCCCCCCCC } \\
\mathrm{E} & \\
\mathrm{S} & \end{array}$ & $\begin{array}{l}4115150 \\
4116850 \\
1110+40 \\
1109+00\end{array}$ & $\begin{array}{l}\mathrm{C} \\
09 \mathrm{~S} \\
04 \mathrm{~W} \\
12\end{array}$ & $\mathrm{AB}$ & $\mathrm{E}$ \\
\hline 4 APL-1 & LOWE RIVER & & & & $\mathrm{B}$ & & \\
\hline$* 784.0$ & (Tributary LOWE RIVER) & DV;SS & $\begin{array}{l}Y_{1}^{\prime} \\
E_{i}^{\prime}\end{array}$ & 4139675 & $\begin{array}{l}\mathrm{C} \\
09 \mathrm{~S}\end{array}$ & $\mathrm{~B}$ & $\mathbf{J}$ \\
\hline 4 & $\begin{array}{l}\text { TAPS B/G; CMP } \\
\text { Stream } \# 2 \text { of } 4\end{array}$ & & S! & $871+43$ & $\begin{array}{l}04 \mathrm{~W} \\
34\end{array}$ & & \\
\hline$* 784.0$ & (Tributary LOWE RIVER) & DV;SS & $\begin{array}{l}Y ! \\
\end{array}$ & 4139675 & $\begin{array}{l}\mathrm{C} \\
09 \mathrm{~S}\end{array}$ & & J \\
\hline 4 & $\begin{array}{l}\text { TAPS B/G; CMP } \\
\text { Stream } \# 3 \text { of } 4\end{array}$ & & $S i$ & $871+43$ & $\begin{array}{l}04 \mathrm{~W} \\
34\end{array}$ & & \\
\hline $\begin{array}{r}784.3 \\
4\end{array}$ & $\begin{array}{l}\text { BROWNS CREEK } \\
\text { TAPS B/G; BLOCKPOINT }\end{array}$ & $\mathrm{DV}$ & & & $\begin{array}{ll}\mathrm{C} \\
09 \mathrm{~S} \\
04 \mathrm{~W} \\
34 \\
\end{array}$ & & $\mathbf{J}$ \\
\hline
\end{tabular}


USDOI BLM PMO OPEN FILE REPORT - TAPS FISH STREAMS

\begin{tabular}{lll}
\hline MP & $:$ STREAM NAME(s) \\
$\bar{A} / \mathrm{S}$ & $:$ Comments
\end{tabular}

: FISH SPECIES

: A : JaFeMrApMaJuJlAuSeOcNoDe

: D : PERIOD OF SENSITIVITY

: A :

: D :
07/30/03 PAGE 40 of 41

:FIELD : MER : REFER

:STATION : T : --ENCE

: G-5 : R

: SEC

\begin{tabular}{|c|c|c|c|c|c|c|c|c|}
\hline $\begin{array}{c}* 784.8 \\
3\end{array}$ & $\begin{array}{l}\text { (Clear Stream) } \\
\text { TAPS B/G; CMP }\end{array}$ & $\begin{array}{l}\text { DV; PS; } \\
\text { SS }\end{array}$ & $\begin{array}{l}Y \vdots \\
E_{1}^{\vdots} \\
S\end{array}$ & CCCCSSSSSSSSCCCCCCCCCCCC & $\begin{array}{r}4143700 \\
4145200 \\
831+00 \\
816+66 \\
\end{array}$ & $\begin{array}{l}\mathrm{C} \\
09 \mathrm{~S} \\
04 \mathrm{~W} \\
33\end{array}$ & $\mathrm{AB}$ & $E$ \\
\hline $\begin{array}{r}787.7 \\
3\end{array}$ & $\begin{array}{l}\text { UNNAMED CREEK } \\
\text { TAPS B/G; Bridge }\end{array}$ & DV;SS & & CCCCSSSSSSSSCCCCCCCCCCCC & & $\begin{array}{l}\mathrm{C} \\
09 \mathrm{~S} \\
04 \mathrm{~W} \\
33\end{array}$ & & $\mathrm{~J}$ \\
\hline $\begin{array}{c}* 789.0 \\
3\end{array}$ & $\begin{array}{l}\text { CANYON SLOUGH } \\
\text { TAPS B/G; CMP }\end{array}$ & $\begin{array}{l}\text { DS; DV; } \\
\text { PS; RS; SS }\end{array}$ & $\begin{array}{l}Y_{i}^{\prime} \\
E_{\vdots}^{\prime} \\
S\end{array}$ & CCCCSSSSSSSujCCCCCCCCCCC & $\begin{array}{r}4165818 \\
610+00\end{array}$ & $\begin{array}{l}\mathrm{C} \\
09 \mathrm{~S} \\
05 \mathrm{~W} \\
35\end{array}$ & $\mathrm{AB}$ & $E$ \\
\hline $\begin{array}{c}* 789.4 \\
3\end{array}$ & $\begin{array}{l}\text { CANYON SLOUGH } \\
\text { TAPS B/G; CMP }\end{array}$ & $\begin{array}{l}\text { DS; DV; } \\
\text { PS; RS; SS }\end{array}$ & $\begin{array}{l}Y \\
E_{1}^{\prime} \\
S !\end{array}$ & CCCCSSSSSSSujCCCCCCCCCCC & $\begin{array}{r}4167900 \\
577+91\end{array}$ & $\begin{array}{l}\mathrm{C} \\
09 \mathrm{~S} \\
05 \mathrm{~W} \\
35\end{array}$ & $\mathrm{AB}$ & $\mathrm{E}$ \\
\hline $\begin{array}{c}* 790.2 \\
2 \\
\end{array}$ & $\begin{array}{l}\text { Tributary CANYON SLOUGH } \\
\text { TAPS B/G; CMP }\end{array}$ & DV; PS;SS & $\begin{array}{l}\mathrm{Y} \\
\mathrm{E} \\
\mathrm{S}\end{array}$ & CCCCSSSSSSSSjlauCCCCCCCC & $\begin{array}{r}4172303 \\
545+15\end{array}$ & $\begin{array}{l}\mathrm{C} \\
09 \mathrm{~S} \\
05 \mathrm{~W} \\
34\end{array}$ & $\overline{\mathrm{AB}}$ & $\mathrm{E}$ \\
\hline $\begin{array}{c}790.35 \\
2\end{array}$ & $\begin{array}{l}\text { (Salmonberry CK) } \\
\text { TAPS B/G; CMP }\end{array}$ & DV;PS;SS & $\begin{array}{l}\mathrm{Y} \\
\mathrm{E} \\
\mathrm{S}\end{array}$ & CCCCSSSSSSSSjlauCCCCCCCC & $\begin{array}{r}4173035 \\
537+83\end{array}$ & $\begin{array}{l}\mathrm{C} \\
09 \mathrm{~S} \\
05 \mathrm{~W} \\
34 \\
\end{array}$ & $\overline{\mathrm{AB}}$ & $\mathrm{E}$ \\
\hline $\begin{array}{c}* 790.5 \\
2\end{array}$ & $\begin{array}{l}\text { Tributary CANYON SLOUGH } \\
\text { TAPS B/G; LWC }\end{array}$ & DV;SS & $\begin{array}{l}Y \\
\\
\end{array}$ & CCCCSSSSSSSSjlauCCCCCCCC & $\begin{array}{r}4174117 \\
527+01\end{array}$ & $\begin{array}{l}\mathrm{C} \\
09 \mathrm{~S} \\
05 \mathrm{~W} \\
27\end{array}$ & $\overline{\mathrm{AB}}$ & $\mathrm{E}$ \\
\hline $\begin{array}{c}* 790.9 \\
2\end{array}$ & $\begin{array}{l}\text { (Grey Stream) } \\
\text { TAPS B/G; CMP }\end{array}$ & DV; SS & $\begin{array}{l}\mathrm{Y}: \\
\mathrm{E} \\
\mathrm{S}\end{array}$ & CCCCSSSSSSSSjlauCCCCCCCC & $\begin{array}{l}4176212 \\
506+06 \\
\end{array}$ & $\begin{array}{l}\mathrm{C} \\
09 \mathrm{~S} \\
05 \mathrm{~W} \\
28\end{array}$ & $\mathrm{AB}$ & $\mathrm{E}$ \\
\hline $\begin{array}{c}* 791.2 \\
2\end{array}$ & $\begin{array}{l}\text { Tributary CANYON SLOUGH } \\
\text { TAPS B/G; LWC }\end{array}$ & $\begin{array}{l}\text { DS; PS; } \\
\text { SS }\end{array}$ & $\begin{array}{l}Y \\
E_{\vdots}^{\prime} \\
S:\end{array}$ & CCCCSSSSSSjlCCCCCCCCCCCC & $\begin{array}{r}4177685 \\
491+33\end{array}$ & $\begin{array}{l}\mathrm{C} \\
09 \mathrm{~S} \\
05 \mathrm{~W} \\
28\end{array}$ & $\overline{\mathrm{AB}}$ & $E$ \\
\hline $\begin{array}{c}* 791.3 \\
2\end{array}$ & $\begin{array}{l}\text { Tributary CANYON SLOUGH } \\
\text { TAPS B/G; LWC }\end{array}$ & DS; PS;SS & 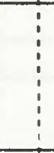 & CCCCSSSSSSjuCCCCCCCCCCCC & $\begin{array}{r}4178086 \\
487+32\end{array}$ & $\begin{array}{l}\mathrm{C} \\
09 \mathrm{~S} \\
05 \mathrm{~W} \\
28 \\
\end{array}$ & $\mathrm{AB}$ & $E$ \\
\hline $\begin{array}{c}* 791.9 \\
2\end{array}$ & $\begin{array}{l}\text { (Tributary LOWE RIVER) } \\
\text { TAPS B/G; LWC }\end{array}$ & SS & $\begin{array}{l}\vec{Y} \\
\mathrm{E} \\
\mathrm{S}\end{array}$ & & $\begin{array}{r}4181441 \\
453+77\end{array}$ & $\begin{array}{l}\mathrm{C} \\
09 \mathrm{~S} \\
05 \mathrm{~W} \\
28 \\
\end{array}$ & $\bar{B}$ & \\
\hline $\begin{array}{c}794.70 \\
2\end{array}$ & $\begin{array}{l}\text { (Abercrombie Slough) } \\
\text { TAPS B/G; CMP }\end{array}$ & $\begin{array}{l}\text { DS; PS; } \\
\text { SS }\end{array}$ & $\begin{array}{l}\mathrm{Y}: \\
\mathrm{E} \\
\mathrm{S} \\
\vdots\end{array}$ & CCCCCCCCCCCCClaCCCCCCCCC & $\begin{array}{r}4196000 \\
308+18\end{array}$ & $\begin{array}{l}\mathrm{C} \\
09 \mathrm{~S} \\
06 \mathrm{~W} \\
24 \\
\end{array}$ & $\mathrm{AB}$ & $\mathrm{E}$ \\
\hline $\begin{array}{r}795.27 \\
2\end{array}$ & $\begin{array}{l}\text { (Abercrombie Slough) } \\
\text { TAPS B/G; CMP }\end{array}$ & DS;PS;SS & $\begin{array}{l}Y \\
\\
\\
S \\
\vdots \\
\vdots\end{array}$ & CCCCCCCCCCCCClaCCCCCCCCC & $\begin{array}{r}4199000 \\
275+10\end{array}$ & $\begin{array}{l}\mathrm{C} \\
09 \mathrm{~S} \\
06 \mathrm{~W} \\
24 \\
\end{array}$ & $\mathrm{AB}$ & $E$ \\
\hline
\end{tabular}




\begin{tabular}{|c|c|c|c|c|}
\hline MP & STREAM NAME(s) & FISH & A : JaFeMrApMaJuJlAuSeOcNoDe & FIELD : MER \\
\hline $\mathrm{A} / \mathrm{S}$ & Comments & : SPECIES & $\begin{array}{l}: \text { D : PERIOD OF SENSITIVITY } \\
\mathrm{A}:\end{array}$ & $\begin{array}{l}: \text { STATION : T } \\
: \quad \text { G-5 }: R\end{array}$ \\
\hline
\end{tabular}

\begin{tabular}{|c|c|c|c|c|c|c|}
\hline $\begin{array}{r}795.87 \\
1\end{array}$ & $\begin{array}{l}\text { ABERCROMBIE GULCH } \\
\text { TAPS B/G; BLOCKPOINT }\end{array}$ & $\begin{array}{l}\text { DS;DV; } \\
\text { PS;SS }\end{array}$ & $\begin{array}{l}\text { CCCCSSSSSjuCCCCCCCCCCCC } \\
\text { E: } \\
S \\
\vdots\end{array}$ & $\begin{array}{r}4202200 \\
252+70\end{array}$ & $\begin{array}{l}\mathrm{C} \\
09 \mathrm{~S} \\
06 \mathrm{~W} \\
23\end{array}$ & $\mathrm{AB}$ \\
\hline $\begin{array}{c}796.63 \\
1\end{array}$ & $\begin{array}{l}\text { (Dayville Flats CK) } \\
\text { Fish downstream of falls } \\
\text { TAPS B/G; CMP }\end{array}$ & $\begin{array}{l}\text { CD; DV; } \\
\text { PS; SS }\end{array}$ & $\begin{array}{l}\text { CCCCSSSSSSjuCCCCCCCCCCCC } \\
\text { Specified as anadromous fish stream } \\
\text { downstream of waterfalls }\end{array}$ & $\begin{array}{r}4206200 \\
205+52\end{array}$ & $\begin{array}{l}\mathrm{C} \\
09 \mathrm{~S} \\
06 \mathrm{~W} \\
15\end{array}$ & $\begin{array}{ll}\mathrm{AB} & \mathrm{E} \\
& \mathrm{J}\end{array}$ \\
\hline $\begin{array}{c}797.02 \\
1\end{array}$ & $\begin{array}{l}\text { (Trickle Creek) } \\
\text { TAPS B/G; LWC }\end{array}$ & PS & CCCCSSSSSSjuCCCCCCCCCCCC & $\begin{array}{r}4208250 \\
176+30\end{array}$ & $\begin{array}{l}\mathrm{C} \\
09 \mathrm{~S} \\
06 \mathrm{~W} \\
15\end{array}$ & B \\
\hline $\begin{array}{c}798.05 \\
1\end{array}$ & $\begin{array}{l}\text { (Solomon Lake Outlet) } \\
\text { Fish downstream of falls } \\
\text { TAPS B/G; BRIDGE }\end{array}$ & $\begin{array}{l}\text { CD; DS; } \\
\text { DV; PS;SS }\end{array}$ & $\begin{array}{l}\text { CCCCSSSSSSjuCCCCCCCCCCCC } \\
\text { Specified as anadromous fish stream } \\
\text { downstream of waterfalls }\end{array}$ & $\begin{array}{r}4213700 \\
126+68\end{array}$ & $\begin{array}{l}\mathrm{C} \\
09 \mathrm{~S} \\
06 \mathrm{~W} \\
21\end{array}$ & ${ }_{\mathrm{H}}^{\mathrm{AB}} \underset{\mathrm{H}^{\mathrm{E}}}{\mathrm{E}}$ \\
\hline $\begin{array}{c}799.74 \\
1\end{array}$ & $\begin{array}{l}\text { ALLISON CREEK } \\
\text { TAPS B/G; BLOCKPOINT }\end{array}$ & $\begin{array}{l}\text { CD; DS; } \\
\text { DV; PS }\end{array}$ & $\begin{array}{lll} & \text { CCCCSSSSS juCCCCCCCCCCCC } \\
E & \end{array}$ & $\begin{array}{r}4222637 \\
35+60\end{array}$ & $\begin{array}{l}\mathrm{C} \\
09 \mathrm{~S} \\
06 \mathrm{~W} \\
18 \\
\end{array}$ & $\mathrm{AB}$ \\
\hline $1 \mathrm{APL}-\mathrm{C}$ & ALLISON CREEK & & & & & B \\
\hline
\end{tabular}




\section{Selected References}

(A)

Alaska Pipeline Office. 1977. Interim report on zones of restricted activity for fish and wildlife along the Trans-Alaska Pipeline. U.S. Dept. of the Interior. Anchorage, AK. (February 16, 1977;37pp).

(B)

Johnson, Richard L. and Julius Rockwell, Jr. (Revised by J. Rockwell, Jr.). 1981. List of streams and other water bodies along the Trans-Alaska oil pipeline route (Fourth Revision: Draft). U.S. Dept. of the Interior, Alaska Pipeline Office, Anchorage, AK (May 1, 1981).

(C)

Elliott, George V. 1980. First interim report on the evaluation of stream crossings and effects of channel modifications on fishery resources along the route of the Trans-Alaska Pipeline. U.S. Fish and Wildlife Service, Anchoragc, AK (June, 1980: 77pp).

(D)

Elliott, George V. 1982. Final report on the evaluation of stream crossings and effects of channel modifications on fishery resources along the route of the Trans-Alaska Pipeline. U.S. Fish and Wildlife Service, Anchorage AK (March 1982;) 110 pp).

(E)

Office of Special Projects. 1982. [no title]. U.S. Bureau of Land Management, Anchorage, AK. (May 6, 1982). [This list is commonly referred to as Ken Hunt's list and includes comments from the Alaska Dept. of Fish and Game].

(F)

Office of the Federal Inspector for ANGTS. 1984. List of fish stream data. Anchorage, AK (February $13,1984 ; 50 \mathrm{pp})$.

(G)

DenBeste, J. and P. McCart. 1984. Catalog of streams associated with the Trans Alaska pipeline System in the northern district. Volume IV. Prepared for Alyeska Pipeline Service Company by Aquatic Environments Inc., Anchorage, AK. (April 1984; 67 pp).

(H)

Roberson, Kenneth. 1985 (letter of 12/18). [Comments on First Edition of 1/1/86]. Alaska Dept. of Fish and Game, Glenallen, AK.

(I)

Alyeska Pipeline Service Co. 1986 (letter of May 12, No. 86-3642) [Comments on First Edition of 1/1/ 86]. Anchorage AK.

Anadromous Fishes

Alaska Department of Fish and Game, Habitat Division. 1985. Catalog of watcrs important for spawning, rearing or migration of anadromous fishes, as revised March 29, 1985 effective May 19, 1985; Regions II, V, and VI. Juncau, AK.

(J)

Gnath, D.G., D.W. Lieb, and M. Wiedmer. 2002. Trans-Alaska Pipeline System 2002 Fish Habitat Survey. Alaska Department of Fish and Game, Habitat and Restoration Division, Technical Report No. 02-07, Anchorage, AK. 


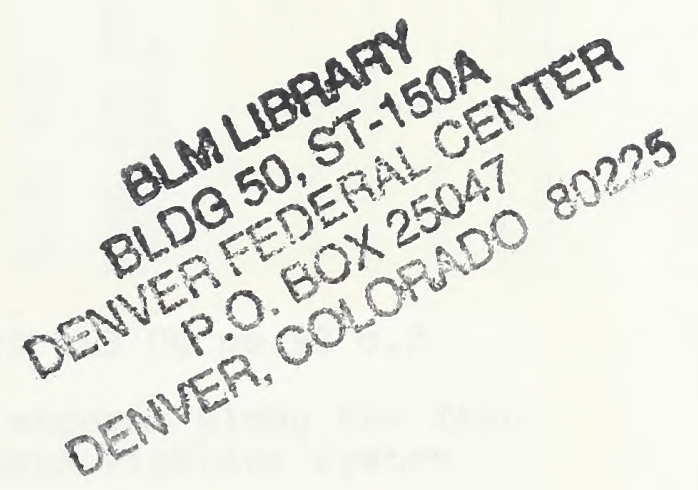




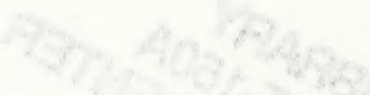




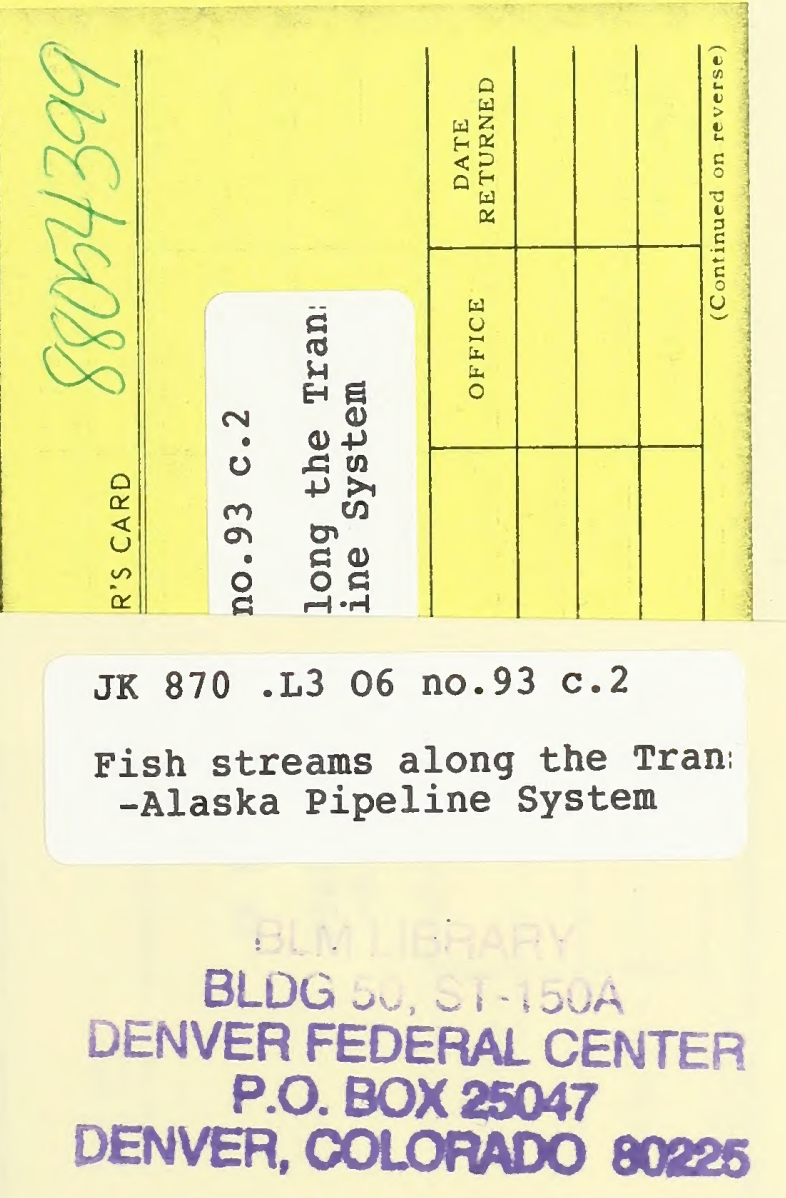


